\title{
Simetrias Globais e Locais em Teorias de Calibre
}

\author{
Bruno Learth Soares
}

TESE APRESENTADA

$\mathrm{AO}$

INSTITUTO DE MATEMÁTICA E ESTATÍSTICA

DA

UNIVERSIDADE DE SÃO PAULO

PARA

OBTENÇÃO DO GRAU DE DOUTOR

EM

CIÊNCIAS

\begin{abstract}
Área de Concentração: Matemática Aplicada Orientador : Prof. Dr. Frank Michael Forger
\end{abstract}

Durante a elaboração deste trabalho o autor recebeu apoio do CNPq, da FAPESP e da CAPES

- São Paulo, 8 de março de 2007 - 


\section{Resumo}

Este trabalho aborda a formulação geométrica das teorias clássicas de calibre, ou Yang-Mills, considerando-as como uma importante classe de modelos que deve ser incluída em qualquer tentativa de estabelecer um formalismo matemático geral para a teoria clássica dos campos. Tal formulação deve vir em (pelo menos) duas variantes: a versão hamiltoniana, que passou por uma fase de desenvolvimento rápido durante os últimos 10-15 anos, levando a o que hoje é conhecido como o "formalismo multissimplético", e a mais tradicional versão lagrangiana utilizada nesta tese. O motivo principal justificando tal investigação é que teorias de calibre constituem os mais importantes exemplos de sistemas dinâmicos que são altamente relevantes na Física e onde a equivalência entre a versão lagrangiana e a versão hamiltoniana, que no caso de sistemas não-singulares é estabelecida pela transformação de Legendre, deixa de ser óbvia, pois teorias de calibre são sistemas degenerados do ponto de vista lagrangiano e são sistemas vinculados do ponto de vista hamiltoniana. Esta propriedade característica das teorias de calibre é uma consequência direta do seu alto grau de simetria, isto é, da sua invariância de calibre. No entanto, numa formulação plenamente geométrica da teoria clássica dos campos, capaz de incorporar situações topologicamente não-triviais, a invariância sob transformações de calibre locais (transformações de calibre de segunda espécie) e, surpreendentemente, até a invariância sob as transformações de simetria globais correspondentes (transformações de calibre de primeira espécie) não pode ser adequadamente descrita em termos de grupos de Lie e suas ações em variedades, mas requer a introdução e o uso sistemático de um novo conceito, a saber, fibrados de grupos de Lie e suas ações em fibrados (sobre a mesma variedade base). A meta principal da presente tese é tomar os primeiros passos no desenvolvimento de ferramentas matemáticas adequadas para lidar com este novo conceito de simetria e, como uma primeira aplicação, dar uma definição clara e simples do procedimento de "acoplamento mínimo" e uma demonstração simples do teorema de Utiyama, segundo o qual lagrangianas para potenciais de calibre (conexões) que são invariantes sob transformações de calibre e de primeira ordem, i.e., dependem apenas dos próprios potenciais de calibre e de suas derivadas parciais até primeira ordem, são necessariamente funções dos campos de calibre (i.e., do tensor de curvatura) invariantes sob as transformações de simetria globais correspondentes. 


\begin{abstract}
This thesis deals with the geometric formulation of classical gauge theories, or Yang-Mills theories, regarded as an important class of models that must be included in any attempt to establish a general mathematical framework for classical field theory. Such a formulation must come in (at least) two variants: the hamiltonian version which has gone through a phase of rapid development during the last 10-15 years, leading to what is now known as the "multisymplectic formalism", and the more traditional lagrangian version studied in this thesis. The main motivation justifying this kind of investigation is that gauge theories constitute the most important examples of dynamical systems that are highly relevant in physics and where the equivalence between the lagrangian and the hamiltonian version, which for non-singular systems is established through the Legendre transformation, is far from obvious, since gauge theories are degenerate systems from the lagrangian point of view and are constrained systems from the hamiltonian point of view. This characteristic property of gauge theories is a direct consequence of their high degree of symmetry, that is, of gauge invariance. However, in a fully geometric formulation of classical field theory, capable of incorporating topologically non-trivial situations, invariance under local gauge transformations (gauge transformations of the second kind) and, surprisingly, even invariance under the corresponding global symmetry transformations (gauge transformations of the first kind) cannot be described adequately in terms of Lie groups and their actions on manifolds but requires the introduction and systematic use of a new concept, namely Lie group bundles and their actions on fiber bundles (over the same base manifold). The main goal of the present thesis is to take the first steps in developing adequate mathematical tools for handling this new concept of symmetry and, as a first application, give a simple clear-cut definition for the prescription of "minimal coupling" and a simple proof of Utiyama's theorem, according to which lagrangians for gauge potentials (connections) that are gauge invariant and of first order, i.e., depend only on the gauge potentials themselves and on their partial derivatives up to first order, are necessarily functions of the gauge field strengths (i.e., the curvature tensor) invariant under the corresponding global symmetry transformations.
\end{abstract}





\section{Conteúdo}

$\begin{array}{ll}\text { Introdução } & 1\end{array}$

1 Simetrias globais e locais na mecânica 11

1.1 Invariância global e invariância local . . . . . . . . . . . . . . . . . 15

1.1 .1 Ação global $(\operatorname{de} G) \ldots \ldots \ldots \ldots$

1.1 .2 Ação local $($ de $T G) \ldots \ldots \ldots \ldots$. . . . . . . . . . . . 17

1.1.3 Invariância local no formalismo lagrangiano . . . . . . . . . . . 19

1.1.4 Invariância local no formalismo hamiltoniano . . . . . . . . . . . 20

1.2 Aplicação momento . . . . . . . . . . . . . . . . . . . . . . . . . . . . . . 21

1.3 Redução simplética . . . . . . . . . . . . . . . . . . . . . . . . . . . . 24

2 Fibrados, Jatos e Conexões $\quad 27$

2.1 Fibrados . . . . . . . . . . . . . . . . . . . . . . 27

2.1 .1 Fibrados gerais . . . . . . . . . . . . . . . 27

2.1.2 Fibrados com grupo estrutural . . . . . . . . . . . . . . . . . . 31

2.1 .3 Fibrados principais . . . . . . . . . . . . . . . . . 32

2.1.4 Fibrados associados . . . . . . . . . . . . . . . . . . . . 34

2.1.5 Estruturas localmente constantes nas fibras . . . . . . . . . . 37

$2.2 \quad J a t o s \ldots \ldots \ldots \ldots \ldots$

$2.2 .1 \quad$ Fibrado vertical . . . . . . . . . . . . . . . . . . . . . . . . . . . 39 
2.2 .2 Fibrados de jatos . . . . . . . . . . . . . . . . . 40

2.2 .3 Grupos de jatos . . . . . . . . . . . . . . . . . . . . 43

2.2.4 Fibrado dos jatos de primeira ordem . . . . . . . . . . . 46

2.2.5 Fibrado dos jatos de segunda ordem (por iteração) . . . . . . . . . 49

2.3 Conexões . . . . . . . . . . . . . . . . . . . . . . . . . . 52

2.3 .1 Conexões gerais . . . . . . . . . . . . . . . . . . . . . 52

2.3 .2 Conexões principais . . . . . . . . . . . . . . . . . . . . . . 54

2.3 .3 Fibrado das conexões . . . . . . . . . . . . . . . . . . . 56

2.3.4 Conexões associadas e derivada covariante . . . . . . . . . . . . 60

2.3.5 Fibrado dos jatos do fibrado das conexões e curvatura . . . . . . . . . 64

2.4 Fibrado dos cojatos . . . . . . . . . . . . . . . . . 66

$\begin{array}{lll}3 & \text { Simetrias globais e locais } & 67\end{array}$

3.1 Fibrados de grupos de Lie . . . . . . . . . . . . . . . . . . . 72

3.2 Ações de fibrados de grupos de Lie . . . . . . . . . . . . . . . . 75

3.3 Ações induzidas . . . . . . . . . . . . . . . . . . . . . . 79

3.3.1 Fibrado das conexões . . . . . . . . . . . . . . . . . . . . . . 83

3.3.2 Fibrado dos jatos de conexões . . . . . . . . . . . . . . . . 84

3.3 .3 Fibrado dos cojatos . . . . . . . . . . . . . . . . . 86

3.4 Grupóides de Lie, ações . . . . . . . . . . . . . . . . . . . . . . . . 87

3.4 .1 Grupóides de Lie . . . . . . . . . . . . . . . . . . . 87

3.4 .2 Ações de grupóides de Lie . . . . . . . . . . . . . . . . 88

3.4 .3 Bisseções . . . . . . . . . . . . . . . . . . . . . . . . . . . . . . 88

3.4 .4 Grupóide de calibre . . . . . . . . . . . . . . . . . . . 89

3.5 Invariância local e global . . . . . . . . . . . . . . . . . . . 89

4 Acoplamento Mínimo e Teorema de Utiyama $\quad 93$

4.1 Acoplamento mínimo . . . . . . . . . . . . . . . . . . . . . . . . . 94

4.2 Teorema de Utiyama . . . . . . . . . . . . . . . . . . . . . . . . . . . 98

$\begin{array}{lr}\text { Conclusão e perspectivas } & 103\end{array}$ 



\section{Introdução}

O presente trabalho visa dar uma contribuição à questão de como implementar, de forma matematicamente correta e rigorosa, o conceito de simetria global e o conceito de simetria local, ou simetria de calibre, na teoria geométrica dos campos.

O conceito de simetria tem sido um dos princípios mais importantes para o desenvolvimento da física teórica no século 20, aplicado numa grande variedade de contextos diferentes. As suas múltiplas facetas podem ser organizadas, pelo menos parcialmente, em termos das seguintes noções.

- Simetrias espaciais ou espaço-temporais / simetrias internas:

Simetrias realizadas por transformações tais como translações, rotações e reflexões no espaço físico tridimensional, translações no tempo ou, no caso da física relativística onde espaço e tempo se fundem no espaço-tempo, transformações de Lorentz costumam ser chamadas de simetrias espaço-temporais. Em contrapartida, simetrias internas são realizadas por transformações num espaço abstrato interno que nada tem a ver com o espaço ou espaço-tempo físico mas constitui o ambiente para as variáveis dinâmicas do modelo sob consideração.

- Simetrias contínuas / simetrias discretas:

Simetrias podem ser distinguidas conforme a natureza do grupo de transformações envolvido: grupos de Lie (conexos) para simetrias tais como translações ou rotações espaciais que são parametrizadas por variáveis contínuas (vetores ou ângulos, no caso), e grupos discretos para simetrias tais como translações e rotações em cristalografia ou reflexões (paridade, inversão temporal, conjugação de cargas etc.). 
- Simetrias globais / simetrias locais:

Uma das mais importantes extensões do conceito tradicional de simetria provém da idéia de que os parâmetros caracterizando um determinado elemento dentro do grupo de simetria pertinente podem depender do instante ou do ponto no espaço-tempo onde a transformação de simetria é executada. Esta noção de "calibrar uma simetria", permitindo que as operações de simetria sejam definidas localmente e não globalmente, ou seja, permitindo que se apliquem transformações diferentes em locais diferentes, é um dos fundamentos das teorias de calibre (ou "gauge theories"), que ocupam uma posição central na teoria dos campos (tanto na clássica como na quântica) e na física das partículas, formando a base do "modelo padrão".

- Simetrias exatas / simetrias quebradas:

Uma simetria é dita quebrada se é válida apenas aproximadamente, i.e., quando há um desvio da simetria exata que no entanto é suficientemente pequeno para que esta ainda permaneça claramente perceptível.

Cabe mencionar, desde já, que existem alguns conceitos de simetria cuja formalização matemática não se enquadra na teoria dos grupos mas é baseada em outros tipos de álgebras, tais como a supersimetria, que usa superálgebras de Lie em vez de álgebras de Lie, ou a simetria quântica descrita por "grupos quânticos", que apesar do nome não são grupos mas são álgebras de Hopf. No entanto, tais generalizações não serão consideradas aqui, pois uma das metas principais deste trabalho é demonstrar que o tipo de simetria encontrada em teorias de calibre requer uma extensão do formalismo matemático em uma direção diferente, levando à noção de um fibrado de grupos de Lie e, mais geralmente, de um grupóide de Lie.

Historicamente, o uso sistemático de princípios de simetria em física no século 20 começa com a teoria da relatividade de Einstein. A relatividade restrita foi baseada numa análise criteriosa da simetria que relaciona as diferentes escolhas possíveis de referenciais inerciais (invariância de Galilei vs. invariância de Lorentz). De forma semelhante, o ponto de partida para a relatividade geral foi a tentativa de Einstein de estender este raciocínio a referenciais não-inerciais, em conjunto com a sua percepção de que tal procedimento estabelece uma ligação profunda com o fenômeno da gravitação, o que o levou a formular os dois princípios que formam a base da relatividade geral: o princípio da covariância geral e o princípio da equivalência. Numa outra direção, argumentos de simetria continuam tendo um papel importante na relatividade geral até hoje pois todas as soluções exatas das equações de Einstein conhecidas possuem grupos de isometrias não-triviais, que são cruciais para reduzir as equações de Einstein a um sistema de equações diferenciais mais simples que pode ser resolvido explicitamente. 
Não obstante a importância de considerações de simetria na relatividade, o impulso decisivo para o seu uso sistemático em física, assim como para o desenvolvimento do seu embasamento matemático, veio do advento da física quântica. Além da aplicação direta de métodos da teoria dos grupos a problemas da mecânica quântica, protagonizada por Hermann Weyl e logo exposta em livros texto de van der Waerden, Weyl e Wigner [32-34,36], uma primeira pedra angular de natureza teórica foi a classificação das partículas elementares em termos de representações unitárias irredutíveis do grupo de Poincaré, devida a Wigner [37]. Numa outra direção, de caráter essencialmente fenomenológico, a teoria dos grupos começou a ser utilizada para classificar degenerescências exatas ou aproximadas entre estados observados na física das partículas. Esta abordagem originou-se com o postulado da invariância das forças nucleares sob o grupo $S U(2)$ de isospin, devido a Heisenberg, e ganhou aceitação geral após a sua extensão ao postulado da invariância das interações fortes sob o grupo $S U(3)$ de "flavor", estabelecido por Gell-Mann e Ne'eman 30 anos depois [16], o que levou ao modelo da estrutura da matéria hoje universalmente aceito, segundo o qual os hadrons (partículas sujeitas às interações fortes) são compostos de quarks.

Uma outra vertente da evolução da física moderna no século 20, na qual princípios de simetria e a teoria dos grupos também desempenham um papel central, foi o desenvolvimento de uma classe de modelos da teoria dos campos hoje conhecida como teorias de calibre (ou "gauge theories"). Mais uma vez, o pioneiro foi Hermann Weyl, que em 1919 propôs tratar transformações de escala no espaço-tempo, que podem ser vistas como efetuando uma recalibragem das unidades em termos das quais medimos distâncias no espaço e no tempo, como simetrias locais, com a esperança de assim poder unificar as duas forças fundamentais conhecidas na época: a eletromagnética e a gravitacional [35]. Esta esperança não se realizou, e a tão desejada unificação não foi alcançada até hoje, mas nasceu a idéia de uma simetria local, que se tornaria extremamente frutífera. De fato, foi o próprio Hermann Weyl que em 1929 aplicou o mesmo princípio não a transformações de escala mas a transformações de fase mais exatamente das fases que aparecem nas funções de onda da mecânica quântica (conceito desconhecido em 1919) - e assim conseguiu deduzir toda a teoria do eletromagnetismo a partir do princípio de deixar uma simetria global se tornar local. Ironicamente, no entanto, o termo "calibre" permaneceu. O próxima passo foi dado por Chen Ning Yang e Robert Mills que em 1954 construíram o primeiro exemplo de uma teoria de calibre não-abeliana, baseada no grupo $S U(2)$ [38], o que logo foi generalizado a um grupo de Lie compacto $G$ qualquer por Ryoyu Utiyama em 1956 [31]. No entanto, o impacto na física das partículas permaneceu limitado até a incorporação do mecanismo de quebra espontânea de simetria (mecanismo de Higgs), a demonstração da renormalizabilidade das teorias de Yang-Mills e, finalmente, a descoberta do fenômeno de liberdade assintótica que, no seu conjunto, foram essenciais para a formulação do que hoje é conhecido como o modelo padrão da física das partículas.

Do ponto de vista matemático, as teorias de calibre também se mostraram bastante frutíferas, devido à estreita relação da sua versão clássica com conceitos básicos da geo- 
metria e topologia diferencial, tais como fibrados, conexões, curvatura, holonomia e classes características, e devido ao impacto que tiveram sobre o desenvolvimento recente desta área, através dos trabalhos de Donaldson, Freedman, Floer e outros.

A teoria clássica dos campos pode ser considerada como uma área da teoria dos sistemas dinâmicos onde as variáveis dinâmicas não são apenas funções do tempo mas também da posição no espaço. Independentemente da questão de se estamos lidando com uma teoria relativística ou não, podemos no caso mais simples considerar campos como funções definidas no espaço-tempo plano de Minkowski $\mathbb{R}^{n}=\mathbb{R} \times \mathbb{R}^{n-1}$ tomando valores reais ou complexos ou, um pouco mais geralmente, tomando valores em um espaço vetorial $\mathbb{E}$ fixo. Naturalmente, nos casos de maior relevância em física, temos $n=4$, mas outros valores de $n$ aparecem em vários contextos, por exemplo no estudo de modelos simplificados com $n<4$ ou na redução dimensional (teorias de Kaluza-Klein) com $n>4$. Neste âmbito, a formalização matemática do conceito de simetria baseia-se na teoria dos grupos e das suas representações. Concretamente, considere a situação em que os campos básicos da teoria correspondem a funções suaves $\varphi$ definidas em $\mathbb{R}^{n}$ a valores em $\mathbb{E}$ e que a dinâmica do modelo é determinada por uma lagrangiana $L$, que pode ser considerada como uma função

$$
L: \mathbb{R}^{n} \times \mathbb{E} \times L\left(\mathbb{R}^{n}, \mathbb{E}\right) \longrightarrow \mathbb{R},
$$

tal que as soluções das equações de movimento da teoria são os pontos estacionários do funcional ação correspondente, definido por

$$
S_{K}[\varphi]=\int_{K} d^{n} x L(x, \varphi(x), \partial \varphi(x)),
$$

para todo subconjunto compacto $K$ de $\mathbb{R}^{n}$, onde $\partial \varphi$ denota o conjunto das (primeiras) derivadas parciais de $\varphi$. Suponha também que o espaço alvo $\mathbb{E}$ vêm munido de uma representação de um grupo de Lie $G$, que escrevemos na forma de uma ação linear

$$
\begin{aligned}
G \times \mathbb{E} & \longrightarrow \mathbb{E} \\
(g, v) & \longmapsto g \cdot v
\end{aligned},
$$

a qual induz uma ação linear

$$
\begin{array}{ccc}
G \times L\left(\mathbb{R}^{n}, \mathbb{E}\right) & \longrightarrow L\left(\mathbb{R}^{n}, \mathbb{E}\right) \\
(g, \alpha) & \longmapsto & g \cdot \alpha
\end{array}
$$

definida por

$$
(g \cdot \alpha)(u)=g \cdot \alpha(u) \quad \text { para } g \in G, \alpha \in L\left(\mathbb{R}^{n}, \mathbb{E}\right), u \in \mathbb{R}^{n}
$$


Em nível infinitesimal, obtém-se uma representação da álgebra de Lie $\mathfrak{g}$ de $G$, que mais uma vez escrevemos na forma de uma ação linear

$$
\begin{aligned}
\mathfrak{g} \times \mathbb{E} & \longrightarrow \mathbb{E} \\
(X, v) & \longmapsto X \cdot v
\end{aligned},
$$

a qual induz uma ação linear

$$
\begin{array}{ccc}
\mathfrak{g} \times L\left(\mathbb{R}^{n}, \mathbb{E}\right) & \longrightarrow L\left(\mathbb{R}^{n}, \mathbb{E}\right) \\
(X, \alpha) & \longmapsto & X \cdot \alpha
\end{array}
$$

definida da mesma maneira que antes:

$$
(X \cdot \alpha)(u)=X \cdot \alpha(u) \quad \text { para } X \in \mathfrak{g}, \alpha \in L\left(\mathbb{R}^{n}, \mathbb{E}\right), u \in \mathbb{R}^{n} .
$$

Diz-se então que a teoria é globalmente $G$-invariante se, para todo subconjunto compacto $K$ de $\mathbb{R}^{n}, S_{K}$ é invariante sob a ação induzida do grupo $G$ no espaço $C^{\infty}\left(\mathbb{R}^{n}, \mathbb{E}\right)$ dos campos, o que é equivalente a exigir que $L$ seja $G$-invariante no sentido de que vale

$$
L(x, g \cdot v, g \cdot \alpha)=L(x, v, \alpha) \quad \text { para } g \in G, x \in \mathbb{R}^{n}, v \in E, \alpha \in L\left(\mathbb{R}^{n}, \mathbb{E}\right) .
$$

De forma análoga, diz-se que a teoria é localmente $G$-invariante se, para todo subconjunto compacto $K$ de $\mathbb{R}^{n}, S_{K}$ é invariante sob a ação induzida do grupo $C^{\infty}\left(\mathbb{R}^{n}, G\right)$ no espaço $C^{\infty}\left(\mathbb{R}^{n}, \mathbb{E}\right)$ dos campos, o que é equivalente a exigir que $L$, além de ser $G$-invariante no sentido já especificado, deve satisfazer a relação

$$
L(x, v, X \cdot \alpha)=L(x, v, \alpha) \quad \text { para } X \in \mathfrak{g}, x \in \mathbb{R}^{n}, v \in E, \alpha \in L\left(\mathbb{R}^{n}, \mathbb{E}\right) .
$$

Isso pode ser deduzido observando-se que para $g \in C^{\infty}\left(\mathbb{R}^{n}, G\right)$ e $\varphi \in C^{\infty}\left(\mathbb{R}^{n}, \mathbb{E}\right)$, vale a relação

$$
\partial_{\mu}(g \cdot \varphi)=g \cdot \partial_{\mu} \varphi+\left(\partial_{\mu} g g^{-1}\right) \cdot(g \cdot \varphi) .
$$

Obviamente, uma lagrangiana satisfazendo tal condição é necessariamente degenerada. A observação básica devida a Weyl é que, apesar da maioria das lagrangianas encontradas na física não ser deste tipo, é possível associar a cada lagrangiana $L_{0}$ globalmente $G$-invariante uma outra lagrangiana $L$ localmente $G$-invariante se introduzirmos um novo campo $A$ que é uma 1-forma suave em $\mathbb{R}^{n}$ a valores em $\mathfrak{g}$ e se exigirmos que sob transformações de calibre dadas por funções suaves $g$ definidas em $\mathbb{R}^{n}$ a valores em $G$, os campos $\varphi$ e $A$ se transformem conforme

$$
\varphi \rightarrow g \cdot \varphi \quad, \quad A_{\mu} \rightarrow g A_{\mu} g^{-1}-\partial_{\mu} g g^{-1}
$$

Explicitamente, definimos

$$
L(x, \varphi(x), \partial \varphi(x), A(x))=L_{0}(x, \varphi(x), D \varphi(x))
$$


onde $D \varphi$ denota o conjunto das (primeiras) derivadas covariantes de $\varphi$, definidas por

$$
D_{\mu} \varphi=\partial_{\mu} \varphi+A_{\mu} \cdot \varphi
$$

e que se transformam conforme

$$
D_{\mu}(g \cdot \varphi)=g \cdot D_{\mu} \varphi
$$

o que justifica o termo "derivada covariante", pois

$$
\begin{aligned}
D_{\mu}(g \cdot \varphi) & =\partial_{\mu}(g \cdot \varphi)+\left(g A_{\mu} g^{-1}-\partial_{\mu} g g^{-1}\right) \cdot(g \cdot \varphi) \\
& =g \cdot \partial_{\mu} \varphi+g \cdot\left(A_{\mu} \cdot \varphi\right)=g \cdot D_{\mu} \varphi .
\end{aligned}
$$

Este procedimento para estender uma simetria global a uma simetria local é chamado de calibragem de uma simetria: requer a introdução de um novo campo $A$, chamado de potencial de calibre, para viabilizar a substituição das derivadas parciais dos campos originais por derivadas covariantes. Diz-se, ainda, que a lagrangiana $L$ é obtida a partir da lagrangiana $L_{0}$ por acoplamento mínimo - uma construção bem conhecida em relatividade geral. É claro que esta prescrição ainda não é completa pois a lagrangiana $L$ contém o campo $A$ mas não contém as suas primeiras derivadas, i.e., o campo $A$ é apenas um multiplicador de Lagrange. Falta acrescentar, como segundo passo, um outro termo $L_{\text {gauge }}$ para converter os potenciais de calibre em variáveis verdadeiramente dinâmicas: este termo é a lagrangiana da eletrodinâmica, no caso abeliano, e a lagrangiana de Yang-Mills, no caso não-abeliano.

$\mathrm{Na}$ teoria geométrica dos campos, o âmbito para a construção descrita acima precisa ser ampliado em várias direções. A primeira extensão deste tipo consiste em substituir o espaço-tempo plano de Minkowski $\mathbb{R}^{n}$ por um espaço-tempo geral, i.e., uma variedade lorentziana $M$ de dimensão $n$. Também podemos substituir o espaço vetorial alvo $\mathbb{E}$ por uma variedade alvo $Q$, como ocorre, por exemplo, nos modelos sigma. E finalmente, é importante permitir que campos sejam representados por seções de um fibrado $E$ sobre $M$, cuja fibra típica é o espaço vetorial alvo $\mathbb{E}$ (no caso de fibrados vetoriais) ou a variedade alvo $Q$ (no caso de fibrados gerais). Este fibrado pode ser topologicamente não-trivial, como ocorre em diversas situações de interesse (no estudo de instantons e de monopolos, por exemplo), mas é importante ressaltar que mesmo se ele for topologicamente trivial, como é necessariamente o caso quando o espaço-tempo $M$ for topologicamente trivial, não existe em geral nenhuma trivialização preferida, sendo que a possibilidade de mudança de trivialização está intimamente ligada à possibilidade de efetuar transformações de calibre.

Infelizmente, a implementação matemática do conceito de simetria na teoria clássica dos campos delineada acima é conceitualmente inadequada para lidar com estas generalizações. Cabe mencionar que existe um método, já considerado padrão, para incorporar simetrias locais no contexto geométrico geral. Para tanto, é conveniente considerar o fibrado $E$ sobre $M$ 
como sendo um fibrado associado a algum fibrado principal $P$ sobre $M$ com grupo estrutural $G$ em relação a uma ação dada de $G$ na fibra típica $Q$,

$$
E=P \times_{G} Q
$$

o que permite duas interpretações diferentes do conceito de transformações de calibre:

- Do ponto de vista passivo, uma transformação de calibre corresponde a uma mudança de trivialização local (equivariante), ou seção local, do fibrado principal $P$, que induz uma mudança de trivialização local em qualquer fibrado associado: dado um aberto $U$ de $M$ sobre o qual $P$ é trivial, ela pode ser representada por uma função $g \in C^{\infty}(U, G)$ que leva uma seção $\sigma$ de $P$ sobre $U$ a outra seção $\tau$ de $P$ sobre $U$ conforme

$$
\tau(x)=\sigma(x) \cdot g(x) \quad \text { para } x \in U
$$

- Do ponto de vista ativo, uma transformação de calibre corresponde a um automorfismo estrito $f: P \rightarrow P$ do fibrado principal $P$, que induz um automorfismo estrito de qualquer fibrado associado: pode ser representada por uma função equivariante $g \in C_{E}^{\infty}(P, G)$ conforme

$$
f(p)=p \cdot g(p) \quad \text { para } p \in P .
$$

Obviamente, as duas interpretações se reduzem à definição anterior se o fibrado $P$ for trivial, sendo que a segunda apresenta a vantagem de ser de natureza global e ainda de admitir a incorporação de simetrias espaço-temporais, representadas por difeomorfismos de $M$, quando removemos a restrição do automorfismo $f$ ser estrito (i.e., de induzir a identidade sobre a variedade base $M)$. No entanto, o método não permite incorporar as simetrias globais, pelo menos não com o mesmo grau de elegância, pois não existe a noção de um automorfismo constante de um fibrado principal não-trivial, e mesmo quando o fibrado principal for trivial, tal noção dependeria da escolha da trivialização e portanto não possui nenhum significado invariante. Isso significa que, num contexto geométrico, devemos deixar de considerar o grupo de simetrias globais como um subgrupo do grupo de simetrias locais. No máximo, pode ser visto como um grupo quociente, a saber o quociente do grupo dos automorfismos estritos de $P$ pelo subgrupo dos automorfismos estritos de $P$ que deixam uma determinada fibra de $P$ invariante, mas até nesta interpretação ainda existe um grau de arbitrariedade provindo da escolha desta fibra, sendo que os subgrupos associados a duas fibras diferentes são conjugados. Em resumo, enfrentamos o problema de que simplesmente não existe nenhuma maneira natural de deixar o grupo estrutural $G$ agir no fibrado $E$ e portanto não sabemos como ele age nos campos da teoria, o que nos impede de definir o que significa invariância da teoria sob simetrias globais. 
Além deste problema, as abordagens descritas acima apresentam uma outra grande inconveniência: para definir o conceito de simetria local, usam-se grupos tais como $C^{\infty}\left(\mathbb{R}^{n}, G\right)$ ou $C_{E}^{\infty}(P, G)$ que, como variedades, são variedades de Fréchet de dimensão infinita, cujo manuseio requer superar enormes dificuldades matemáticas. Além disso, o método tende a camuflar o mais consagrado princípio da teoria dos campos, que é o princípio de localidade, segundo o qual nada que acontece em uma determinada região do espaço-tempo pode influenciar o que acontece em outra região do espaço-tempo causalmente disjunta. Seria altamente desejável encontrar uma formulação dos princípios de simetria global e de simetria local na teoria geométrica dos campos que envolva apenas variedades, fibrados, grupos, álgebras etc. de dimensão finita, evitando assim as complicações de análise funcional envolvidas no tratamento de objetos de dimensão infinita e, ao mesmo tempo, permitindo uma implementação direta e imediata do princípio de localidade.

A meta principal desta tese é apresentar uma formulação alternativa do conceito de simetria que satisfaz todos estes requerimentos. Brevemente, ela é baseada na idéia de que, se lidamos com fibrados, as simetrias também devem ser descritas em termos de fibrados. Portanto, o objeto central, tanto no caso de simetrias globais como no de simetrias locais, não é um grupo de Lie agindo numa variedade (ambos de dimensão infinita no caso de simetrias locais), mas um fibrado de grupos de Lie $^{1}$ sobre o espaço-tempo agindo nos diversos fibrados sobre o espaço-tempo onde "vivem" os campos da teoria. Mais geralmente ainda, para unificar simetrias internas e simetrias espaço-temporais, o objeto fundamental é um grupóide de Lie. Em nível infinitesimal, temos o correspondente fibrado de álgebras de Lie e, mais geralmente, o correspondente algebróide de Lie.

Esta tese será organizada da seguinte forma:

No Capítulo 1, analisamos como funciona no caso específico da mecânica tudo o que será desenvolvido nesta tese.

No Capítulo 2, apresentamos conceitos centrais da geometria diferencial que são básicos para toda a teoria a ser desenvolvida nos capítulos seguintes: fibrados, jatos e conexões. Estas noções são fundamentais pois o nosso principal objetivo é estudar as teorias de calibre no contexto da formulação geométrica da teoria clássica dos campos. Este formalismo tem a grande virtude de permitir abordar questões da teoria clássica dos campos não apenas com absoluto rigor matemático, mas também de maneira covariante (independente da escolha de coordenadas), local (trabalha-se com as variáveis dos campos e suas derivadas em pontos do espaço-tempo e não com campos definidos em subvariedades inteiras, como na formulação usual do problema de Cauchy, sendo que a integração sobre hipersuperfícies ou outras subvariedades é adiada até o último passo) e em dimensão finita.

$\mathrm{Na}$ formulação geométrica da teoria clássica dos campos, o ponto de partida consiste na escolha de um fibrado de configuração, que é o fibrado sobre o espaço-tempo cujas seções

\footnotetext{
${ }^{1}$ Este conceito não deve ser confundido com o de um fibrado principal.
} 
representam os campos básicos da teoria em questão. ${ }^{2}$ A partir dele, constrõem-se o correspondente fibrado dos jatos e o correspondente fibrado dos cojatos, ambos de primeira ordem: o primeiro é o fibrado sobre o espaço-tempo cujas seções representam as primeiras derivadas dos campos básicos e portanto ele constitui o domínio do formalismo lagrangiano, enquanto que o segundo é o seu dual afim e constitui o domínio do formalismo hamiltoniano. ${ }^{3}$

O nosso estudo aqui se concentrará no formalismo lagrangiano para teorias de calibre, que constituem talvez a mais importante classe de modelos da teoria clássica dos campos onde uma abordagem geométrica é, no mínimo, conveniente. Teorias de calibre contêm como campos básicos campos de matéria e campos de calibre, ou campos de Yang-Mills, sendo que em termos do fibrado principal $P$ sobre o espaço-tempo $M$ (com grupo estrutural $G$, digamos) cuja escolha constitui um dos ingredientes para a especificação do modelo, o fibrado de configuração para os campos de matéria é um fibrado associado $P \times_{G} Q$ enquanto que o fibrado de configuração para os próprios campos de calibre é o fibrado $C P$ das conexões principais em $P$, pois as seções globais deste fibrado podem ser identificadas com as conexões principais em $P$. CP é um fibrado afim sobre o espaço-tempo $M$ e algumas de suas mais importantes propriedades, assim como algumas propriedades do seu fibrado de jatos $J(C P)$ já foram objetos de certo estudo (ver [9, 12,14, 19,23]).

O Capítulo 3 é a parte central desta tese. Nele, mostramos que a noção de invariância sob transformações de calibre pode ser formulada utilizando apenas objetos de dimensão finita. As transformações de calibre costumam ser definidas como os elementos do grupo $\mathrm{Gau}(P)$ dos automorfismos de $P$ que cobrem a identidade de $M$. Existem muitos estudos acerca das propriedades deste grupo e de sua ação sobre o espaço $\Gamma\left(P \times_{G} Q\right)$ das seções de $P \times_{G} Q$ e o espaço $\Gamma(C P)$ das seções de $C P$ (veja, por exemplo, [28] e suas referências), mas o fato de todos estes objetos terem dimensão infinita acarreta enormes dificuldades técnicas. No entanto, usando o fato de que transformações de calibre, assim como os diversos tipos de campos nos quais elas agem, são seções de fibrados sobre o espaço-tempo, existe a possibilidade, até então pouco explorada, de "espalhar" estes objetos pelo espaço-tempo, no intuito de substituir construções formais em dimensão infinita por construções equivalentes que envolvem apenas objetos de dimensão finita, localizados em cada ponto do espaçotempo. Assim, podemos formular a condição de invariância de calibre diretamente no nível da lagrangiana, em termos do conceito de um fibrado de grupos de Lie e de uma ação de um fibrado de grupos de Lie em um fibrado, ambos sobre a mesma variedade base.

No Capítulo 4, apresentamos neste contexto o procedimento de acoplamento mínimo, já empregado por Hermann Weyl em sua derivação do eletromagnetismo da mecânica quântica,

\footnotetext{
2Matematicamente, a distinção entre "campos básicos" e "campos compostos" (tais como polinômios nos campos básicos e suas derivadas) é um tanto vaga, e sempre há um certo grau de convenção na escolha de quais campos devem ser considerados básicos e quais compostos.

${ }^{3}$ Isso significa que consideramos apenas teorias de primeira ordem, como já é feito no caso da formulação geométrica da mecânica $[1,3]$, pois estas predominam nas aplicações à física.
} 
que constitui uma construção explícita, a partir de uma lagrangiana globalmente invariante, de uma lagrangiana localmente invariante que depende não apenas do(s) campo(s) de matéria aparecendo na lagrangiana original mas também de um campo de calibre que aparece como "multiplicador de Lagrange", i.e., sem dinâmica própria. Também provamos o teorema de Utiyama, que caracteriza as lagrangianas em $J(C P)$ invariantes sob transformações de calibre como sendo aqueles que dependem apenas da curvatura. Formulamos ainda uma extensão deste teorema, que consiste em uma espécie de unificação dele com o acoplamento mínimo em teorias para campos de matéria e de calibre dinâmicos.

Provamos estes resultados não apenas para ver o formalismo desenvolvido no Capítulo 3 em funcionamento, mas principalmente porque eles serão essenciais para a compreensão da subsequente passagem ao formalismo hamiltoniano onde, no âmbito da área emergente da geometria multissimplética, poderemos introduzir o análogo da aplicação momento e desenvolver um procedimento de redução multissimplética. Cabe ressaltar que o presente trabalho foi desenvolvido tendo estes temas como meta, e já existem alguns resultados preliminares nesta direção, mas como será comentado na seção sobre conclusões e perspectivas que consta do final da tese, conseguimos até o presente apenas construir uma base sólida para um estudo mais aprofundado do formalismo multissimplético em teorias de calibre, que pretendemos continuar no futuro. 
CAPÍTUlo 1

\section{Simetrias globais e locais na mecânica}

A nossa meta neste primeiro capítulo é apresentar uma formulação do conceito de simetria na mecânica que contemple sistemas não-autônomos e permita incluir, de forma natural, a noção de "simetria local" que aparece em teorias de calibre. Trata-se de uma generalização substancial da noção tradicional de simetria em sistemas dinâmicos autônomos, que para fins de distinção será chamada de "simetria global", como é de costume em física.

Para colocar o problema na sua devida perspectiva, começaremos por lembrar a formulação tradicional de simetrias em sistemas dinâmicos conservativos autônomos. Na sua grande maioria, tais sistemas admitem duas formulações diferentes, mas equivalentes: uma lagrangiana e uma hamiltoniana, ambas baseadas na existência de um "espaço das configurações". Este espaço das configurações é uma variedade que aqui será denotada por $Q$ : seus sistemas de coordenadas locais $\left(q^{i}\right)$ são conhecidos na mecânica como "coordenadas generalizadas". Para definir um sistema dinâmico (conservativo e autônomo) com espaço das configurações $Q$, requer-se, como ponto de partida, ou a escolha de uma função lagrangiana $L$ sobre o fibrado tangente $T Q$ de $Q$, que é interpretado como "espaço das velocidades" (com sistemas de coordenadas locais induzidas $\left(q^{i}, \dot{q}^{i}\right)$ ), ou de uma função hamiltoniana $H$ sobre o fibrado cotangente $T^{*} Q$ de $Q$, que é interpretado como "espaço dos momentos" (com sistemas de coordenadas locais induzidas $\left.\left(q^{i}, p_{i}\right)\right)$, sendo que estas funções providenciam as equações de movimento do sistema. No caso mais simples de sistemas não-degenerados, a equivalência entre as duas formulações é estabelecida pela transformação de Legendre. Neste contexto, simetrias são descritas pela ação de um grupo de Lie $G$ sobre o espaço das configurações $Q$ tal que a lagrangiana $L$ e a hamiltoniana $H$ são invariantes sob as ações induzidas de $G$ sobre $T Q$ e sobre $T^{*} Q$, respectivamente.

Como é bem conhecido, este arcabouço admite generalizações em várias direções diferentes. Por exemplo, na mecânica lagrangiana, podemos substituir o fibrado tangente $T Q$ de $Q$ por um subfibrado vetorial não-integrável, o que permite formular a dinâmica de sistemas com vínculos não-holônomos. De forma semelhante, na mecânica hamiltoniana, 
podemos substituir o fibrado cotangente $T^{*} Q$ de $Q$ por uma variedade simplética ou até uma variedade de Poisson, o que permite, entre muitas outras coisas, formular a dinâmica de uma partícula clássica com spin semi-inteiro [30], e podemos ainda considerar ações simpléticas de grupos de Lie ou até ações de Lie-Poisson de grupos de Lie-Poisson [6], que têm papel importante na teoria dos sistemas integráveis. No entanto, a generalização que pretendemos apresentar aqui segue um rumo diferente e, do ponto de vista da abrangência das aplicações na física, talvez mais importante ainda.

O ponto de partida para essa generalização é a observação de que o formalismo para sistemas dinâmicos conservativos autônomos descrito acima é extremamente limitado, pois não contempla adequadamente o papel da variável tempo. Na verdade, ele se baseia implicitamente no axioma do tempo absoluto de Newton, conforme o qual "o tempo absoluto, verdadeiro e matemático, por sua própria natureza, flui uniformemente sem relação a nenhum fator externo", tanto que a variável $t$ fica completamente divorciada das coordenadas $q^{i}$ e $\dot{q}^{i}$ ou $p_{i}$ "internas" que servem para descrever os possíveis estados do sistema. No entanto, desde o advento da teoria da relatividade, sabemos que o mundo não é assim: a variável tempo, uma vez que pode ser submetida a transformações não-triviais que refletem mudanças de referencial ou observador, foi rebaixada de um parâmetro canônico absoluto para uma mera coordenada no espaço-tempo, semelhante às coordenadas espaciais usadas para medir posições. Em consequência disso, a interpretação de curvas como trajetórias de partículas também sofreu uma mudança radical: em vez de curvas no espaço para os quais o tempo absoluto serve como parâmetro canônico, temos "linhas universo" no espaço-tempo cujo parâmetro é arbitrário e não possui nenhuma interpretação física natural, enquanto que a variável tempo se junta às coordenadas espaciais que fazem parte das coordenadas $q^{i}$ para o espaço das configurações.

Mas mesmo no âmbito da mecânica clássica não-relativística, existe (pelo menos) um outro motivo para considerar este tipo de junção da variável tempo $t$ às coordenadas generalizadas $q^{i}$ : a mecânica de sistemas não-autônomos, onde a lagrangiana $L$ e a hamiltoniana $H$ dependem explicitamente do tempo. Normalmente, tal dependência é o resultado do acoplamento do sistema a fontes externas que não são estáticas: um exemplo típico seria um sistema de partículas carregadas sujeito a radiação eletromagnética. Para descrever tais sistemas, faz-se necessário substituir o espaço das configurações $Q$ pelo "espaço das configurações estendido" $\mathbb{R} \times Q$ e, consequentemente, o espaço das velocidades $T Q$ pelo "espaço das velocidades estendido" $\mathbb{R} \times T Q$ e o espaço dos momentos $T^{*} Q$ pelo "espaço dos momentos estendido (ou simplesmente estendido)" $\mathbb{R} \times T^{*} Q$, sendo que $L$ e $H$ são agora funções sobre $\mathbb{R} \times T Q$ e sobre $\mathbb{R} \times T^{*} Q$, respectivamente. No último caso, como já foi observado por É. Cartan, é conveniente considerar uma extensão adicional, a saber o "espaço dos momentos duplamente estendido" $\mathbb{R} \times T^{*} Q \times \mathbb{R}$, sendo que a coordenada adicional $e$ aqui introduzida desempenha o papel de uma variável energia. A vantagem principal deste procedimento é que $\mathbb{R} \times T^{*} Q \times \mathbb{R}$, como o próprio fibrado cotangente $T^{*} Q$, é uma variedade 
simplética exata, com forma canônica

$$
\theta=p_{i} d q^{i}+e d t
$$

e forma simplética

$$
\omega=d q^{i} \wedge d p_{i}-d e \wedge d t
$$

enquanto que $\mathbb{R} \times T^{*} Q$ é apenas uma variedade de contato. Na verdade, como veremos a seguir, o conjunto dessas duas extensões desempenha um papel importante no formalismo hamiltoniano.

Como ponto de partida, notamos que o espaço dos momentos duplamente estendido $\mathbb{R} \times T^{*} Q \times \mathbb{R}$ pode ser considerado como um fibrado (em linhas) sobre o espaço dos momentos simplesmente estendido $\mathbb{R} \times T^{*} Q$, o que permite uma reinterpretação da hamiltoniana como uma seção $\mathcal{H}$ deste fibrado, em vez de uma função $H$ sobre a sua base, sendo que os dois objetos se relacionam da forma óbvia:

$$
\mathcal{H}(t, q, p)=(t, q, p,-H(t, q, p))
$$

(O sinal é meramente uma questão de convenção.) Logo, podemos introduzir a 1-forma $\theta_{\mathcal{H}}$ e a 2 -forma $\omega_{\mathcal{H}}$ sobre $\mathbb{R} \times T^{*} Q$ obtidas por "pull-back" de $\theta$ e de $\omega$, i.e.,

$$
\theta_{\mathcal{H}}=p_{i} d q^{i}-H d t
$$

$\mathrm{e}$

$$
\omega_{\mathcal{H}}=d q^{i} \wedge d p_{i}+\frac{\partial H}{\partial q^{i}} d q^{i} \wedge d t+\frac{\partial H}{\partial p_{i}} d p_{i} \wedge d t
$$

O análogo destas formas no âmbito lagrangiano são as formas de Poincaré-Cartan

$$
\theta_{\mathcal{L}}=\frac{\partial L}{\partial \dot{q}^{i}} d q^{i}+\left(L-\frac{\partial L}{\partial \dot{q}^{i}} \dot{q}^{i}\right) d t
$$

e

$$
\begin{aligned}
\omega_{\mathcal{L}}= & \frac{\partial^{2} L}{\partial q^{j} \partial \dot{q}^{i}} d q^{i} \wedge d q^{j}+\frac{\partial^{2} L}{\partial \dot{q}^{j} \partial \dot{q}^{i}} d q^{i} \wedge d \dot{q}^{j} \\
& +\left(\frac{\partial^{2} L}{\partial t \partial \dot{q}^{i}}-\frac{\partial L}{\partial q^{i}}+\frac{\partial^{2} L}{\partial q^{i} \partial \dot{q}^{j}} \dot{q}^{j}\right) d q^{i} \wedge d t+\left(\frac{\partial^{2} L}{\partial \dot{q}^{i} \partial \dot{q}^{j}} \dot{q}^{j}\right) d \dot{q}^{i} \wedge d t
\end{aligned}
$$

que podem ser obtidas a partir das anteriores por "pull-back" com a transformação de Legendre. Como é bem conhecido, o funcional ação $S$ usado para escrever as equações de movimento do sistema como resultado de um princípio variacional pode, em ambos os casos, ser definido por integração de $\theta_{\mathcal{L}}$ ou $\theta_{\mathcal{H}}$ ao longo de curvas. De fato, denotando por $I$ o 
intervalo de definição das possíveis curvas solução a serem consideradas, temos no formalismo lagrangiano

$$
\begin{gathered}
S_{I}[\mathbf{q}]=\int_{I}(\mathbf{q}, \dot{\mathbf{q}})^{*} \theta_{\mathcal{L}}=\int_{I} d t L(t, \mathbf{q}(t), \dot{\mathbf{q}}(t)) \\
\text { para } \mathbf{q} \in C^{\infty}(I, Q)
\end{gathered}
$$

e no formalismo hamiltoniano

$$
\begin{gathered}
S_{I}[\mathbf{q}, \mathbf{p}]=\int_{I}(\mathbf{q}, \mathbf{p})^{*} \theta_{\mathcal{H}}=\int_{I} d t(\langle\mathbf{p}(t), \dot{\mathbf{q}}(t)\rangle-H(t, \mathbf{q}(t), \mathbf{p}(t))) \\
\operatorname{para}(\mathbf{q}, \mathbf{p}) \in C^{\infty}\left(I, T^{*} Q\right)
\end{gathered}
$$

Um outro mérito da extensão descrita acima, e talvez o mais importante, é que ela permite a generalização de simetrias globais para simetrias locais. O ponto de partida para ambas é uma ação de um grupo de Lie $G$ no espaço das configurações $Q$ que, como já foi observado anteriormente, induz ações de $G$ em $T Q$ e em $T^{*} Q$. Obviamente, deixando $G$ agir trivialmente sobre $\mathbb{R}$, isso fornece ações de $G$ em todos os espaços estendidos, e diz-se que a lagrangiana $L$ e a hamiltoniana $H$ são "globalmente $G$-invariantes" se são invariantes sob essa ação de $G$ no espaço estendido pertinente. A idéia básica de "calibrar" essa simetria e assim transformá-la em uma simetria local, é deixar o elemento do grupo $G$ que age em cada instante $t$ depender de $t$.

O método usualmente adotado para concretizar esta idéia no âmbito lagrangiano consiste em considerar o grupo $C^{\infty}(I, G)$ de curvas em $G$ e suas ações sobre os espaços $C^{\infty}(I, Q)$ e $C^{\infty}(I, T Q)$ de curvas em $Q$ e em $T Q$, respectivamente, onde $I$ é um intervalo fixo de definição em $\mathbb{R}$ que não costuma ser especificado, exigindo-se que o funcional ação $S$ definido por integração de $L$ sobre $I$ ao longo de cada curva seja invariante sob esta ação. No entanto, esta abordagem tem uma série de defeitos, tais como:

- a necessidade de escolher um intervalo $I$ de definição "a priori", sendo que em geral, cada solução das equações de movimento tem o seu próprio intervalo máximo de definição, que depende das condições iniciais e pode ser menor do que o intervalo $I$ escolhido;

- a necessidade de escolher um determinado espaço de curvas, sendo que poderia-se considerar outras opções, além de curvas suaves;

- a dificuldade matemática de lidar com "variedades" ou "grupos de Lie" de dimensão infinita, tais como $C^{\infty}(I, Q)$ ou $C^{\infty}(I, G)$;

- a dificuldade de implementar essa abordagem no formalismo hamiltoniano. 
Tendo em vista estes problemas, é altamente desejável encontrar um critério para que um sistema lagrangiano ou hamiltoniano seja "localmente $G$-invariante", diretamente em termos da lagrangiana $L$ ou hamiltoniana $H$ e não apenas do funcional ação $S$, e empregando apenas objetos de dimensão finita. Este critério é surpreendentemente simples, envolvendo as ações induzidas pela ação dada de $G$ em $Q$, não de $G$ mas de $T G$, o fibrado tangente de $G$, que também é um grupo de Lie, no fibrado tangente $T Q$ de $Q$ e no seu dual afim $T^{\star} Q \cong T^{*} Q \times \mathbb{R}$. $^{1}$ Os detalhes serão apresentados na próxima seção.

\subsection{Invariância global e invariância local}

\subsubsection{Ação global (de $G$ )}

Seja $Q$ uma variedade que servirá como espaço das configurações do sistema dinâmico sob consideração e cujos sistemas de coordenadas locais $\left(q^{i}\right)$ são as "coordenadas generalizadas" da mecânica. Para descrever possíveis simetrias de um tal sistema, introduzimos um grupo de simetria, que é um grupo de Lie $G$ com álgebra de Lie $\mathfrak{g}$, junto com uma ação

$$
\begin{aligned}
G \times Q & \longrightarrow Q \\
(g, q) & \longmapsto g \cdot q
\end{aligned}
$$

de $G$ em $Q$. Em nível infinitesimal, esta induz uma "representação de $\mathfrak{g}$ por campos fundamentais sobre $Q$ ", ou seja, um antihomomorfismo de álgebras de $\mathrm{Lie}^{2}$

$$
\begin{gathered}
\mathfrak{g} \longrightarrow \mathfrak{X}(Q) \\
X \longmapsto X_{Q}
\end{gathered}
$$

onde o termo "antihomomorfismo" significa que vale

$$
\left[X_{Q}, Y_{Q}\right]=-[X, Y]_{Q} \quad \text { para } X, Y \in \mathfrak{g},
$$

sendo que o campo vetorial $X_{Q} \in \mathfrak{X}(Q)$ associado a um gerador $X \in \mathfrak{g}$ é conhecido como o campo fundamental associado a $X$, definido por

$$
X_{Q}(q)=\left.\frac{d}{d t} \exp (t X) \cdot q\right|_{t=0} \quad \text { para } X \in \mathfrak{g},
$$

\footnotetext{
${ }^{1}$ Parece provável que tal critério direto de invariância da hamiltoniana não tenha sido encontrada anteriormente por falta de percepção da necessidade de utilizar o dual afim em conjunto com o dual linear, interpretando o primeiro como fibrado afim em linhas sobre o segundo e a hamiltoniana como seção deste fibrado.

${ }^{2} \mathfrak{X}(Q)$ denota o espaço dos campos vetoriais sobre $Q$, que é uma álgebra de Lie em relação ao colchete de Lie.
} 
e que satisfaz a seguinte propriedade de covariância:

$$
g \cdot X_{Q}\left(g^{-1} \cdot q\right)=(\operatorname{Ad}(g) X)_{Q}(q) \quad \text { para } X \in \mathfrak{g}, g \in G, q \in Q .
$$

Ela também induz uma ação

$$
\begin{array}{ccc}
G \times T Q & \longrightarrow & T Q \\
(g,(q, \dot{q})) & \longmapsto & (g \cdot q, g \cdot \dot{q})
\end{array}
$$

de $G$ no fibrado tangente $T Q$ de $Q$ (que é linear nas fibras de $T Q$ ), definida por

$$
\left.g \cdot \frac{d}{d t} q(t)\right|_{t=0}=\left.\frac{d}{d t}(g \cdot q(t))\right|_{t=0}
$$

e, por dualização, uma ação

$$
\begin{aligned}
G \times T^{*} Q & \longrightarrow T^{*} Q \\
(g,(q, p)) & \longmapsto(g \cdot q, g \cdot p)
\end{aligned}
$$

de $G$ no fibrado cotangente (linear) $T^{*} Q$ de $Q$, definida por

$$
\langle g \cdot p, g \cdot \dot{q}\rangle=\langle p, \dot{q}\rangle \quad \text { para } g \in G, q \in Q, p \in T_{q}^{*} Q, \dot{q} \in T_{q} Q
$$

onde $\langle.,$.$\rangle denota o pareamente natural entre espaço cotangente e espaço tangente. Em nível$ infinitesimal, estas induzem "representações de $\mathfrak{g}$ por campos fundamentais sobre $T Q$ e sobre $T^{*} Q "$, ou seja, antihomomorfismos de álgebras de Lie

$$
\begin{aligned}
& \mathfrak{g} \longrightarrow \mathfrak{X}(T Q) \\
& X \longmapsto X_{T Q}
\end{aligned}
$$

e

$$
\begin{aligned}
\mathfrak{g} & \longrightarrow \mathfrak{X}\left(T^{*} Q\right) \\
X & \longmapsto X_{T^{*} Q}
\end{aligned}
$$

Explicitamente, em termos de coordenadas locais $\left(q^{i}, \dot{q}^{i}\right)$ de $T Q$ e coordenadas locais $\left(q^{i}, p_{i}\right)$ de $T^{*} Q$ induzidas por coordenadas locais $q^{i}$ de $Q$, onde

$$
X_{Q}=X_{Q}^{i} \frac{\partial}{\partial q^{i}},
$$

temos

$$
X_{T Q}=X_{Q}^{i} \frac{\partial}{\partial q^{i}}+\dot{q}^{j} \frac{\partial X_{Q}^{i}}{\partial q^{j}} \frac{\partial}{\partial \dot{q}^{i}}
$$

e

$$
X_{T^{*} Q}=X_{Q}^{i} \frac{\partial}{\partial q^{i}}-p_{j} \frac{\partial X_{Q}^{j}}{\partial q^{i}} \frac{\partial}{\partial p_{i}} .
$$




\subsubsection{Ação local (de $T G)$}

A chave que permite descrever como estender simetrias globais para simetrias locais na mecânica consiste em considerar o fibrado tangente $T G$ de $G$ e aplicar o funtor tangente à multiplicação $G \times G \rightarrow G$ para obter uma aplicação $T G \times T G \rightarrow T G$ que satisfaz todos os axiomas de uma multiplicação de grupo e portanto torna $T G$ um grupo de Lie. ${ }^{3}$ Por outro lado, $T G$ também é um fibrado vetorial sobre $G$ que é globalmente trivial: podemos usar translações (à esquerda ou à direita) por elementos de $G$ para exibir uma trivialização explícita. A seguir, usaremos, por convenção, translações a direita, ou seja, o isomorfismo canônico

$$
\begin{aligned}
& T G \longmapsto \\
&(g, \dot{g}) \cong \times \mathfrak{g} \\
&\left(g, \dot{g} g^{-1}\right)
\end{aligned}
$$

mostrando que $T G$ é realmente o produto semidireto $G \ltimes \mathfrak{g}$ de $G$ e $\mathfrak{g}$ (onde $\mathfrak{g}$ é considerado como grupo abeliano com respeito à adição) em relação à ação de $G$ por automorfismos de $\mathfrak{g}$ definida pela representação adjunta. Em outras palavras, sob o isomorfismo anterior, a multiplicação em $T G$ é dada por

$$
\begin{gathered}
\left(g_{1}, X_{1}\right)\left(g_{2}, X_{2}\right)=\left(g_{1} g_{2}, X_{1}+\operatorname{Ad}\left(g_{1}\right)\left(X_{2}\right)\right) \\
\text { para }\left(g_{1}, X_{1}\right),\left(g_{2}, X_{2}\right) \in G \ltimes \mathfrak{g},
\end{gathered}
$$

a unidade é $(1,0)$ e a inversa é dada por

$$
(g, X)^{-1}=\left(g^{-1},-\operatorname{Ad}\left(g^{-1}\right)(X)\right) \quad \text { para }(g, X) \in G \ltimes \mathfrak{g} .
$$

Obviamente, $T G \cong G \ltimes \mathfrak{g}$ contém dois subgrupos: uma parte global, que é o grupo original $G$, e uma parte local, que é o subgrupo abeliano normal $\mathfrak{g}$. Obviamente, a correspondente álgebra de Lie é então a soma semidireta $\mathfrak{g} \ltimes \mathfrak{g}$ de duas cópias de $\mathfrak{g}$, com comutador dado por

$$
\begin{gathered}
{\left[\left(X_{1}, \xi_{1}\right),\left(X_{2}, \xi_{2}\right)\right]=\left(\left[X_{1}, X_{2}\right],\left[X_{1}, \xi_{2}\right]-\left[X_{2}, \xi_{1}\right]\right)} \\
\operatorname{para}\left(X_{1}, \xi_{1}\right),\left(X_{2}, \xi_{2}\right) \in \mathfrak{g} \ltimes \mathfrak{g} .
\end{gathered}
$$

De forma semelhante, podemos aplicar o funtor tangente à ação $G \times Q \rightarrow Q$ para obter uma aplicação $T G \times T Q \rightarrow T Q$ que satisfaz todos os axiomas de uma ação de grupo. Explicitamente, esta ação

$$
\begin{array}{ccc}
T G \times T Q & \longrightarrow & T Q \\
((g, \dot{g}),(q, \dot{q})) & \longmapsto & (g, \dot{g}) \cdot(q, \dot{q})
\end{array}
$$

\footnotetext{
${ }^{3}$ Aqui, como sempre, usa-se o isomorfismo canônico $T(M \times N) \cong T M \times T N$.
} 
é dada por

$$
\left.\begin{array}{l}
g=g(0), \dot{g}=\left.\frac{d}{d t} g(t)\right|_{t=0} \\
q=q(0), \dot{q}=\left.\frac{d}{d t} q(t)\right|_{t=0}
\end{array}\right\} \Longrightarrow(g, \dot{g}) \cdot(q, \dot{q})=\left.\frac{d}{d t}(g(t) \cdot q(t))\right|_{t=0}
$$

e ela estende a ação (1.15) de $G$ sobre $T Q$. Sob o isomorfismo anterior, ela assume a forma

$$
\begin{gathered}
(g, X) \cdot(q, \dot{q})=\left(g \cdot q, g \cdot \dot{q}+X_{Q}(g \cdot q)\right) \\
\operatorname{para}(g, X) \in G \ltimes \mathfrak{g}, q \in Q, \dot{q} \in T_{q} Q .
\end{gathered}
$$

Observe que esta ação é apenas afim nas fibras de $T Q$ e portanto, por dualização, induz uma ação

$$
\begin{array}{ccc}
T G \times T^{\star} Q & \longrightarrow & T^{\star} Q \\
((g, \dot{g}),(q, p, e)) & \longmapsto & (g, \dot{g}) \cdot(q, p, e)
\end{array}
$$

no fibrado cotangente (afim) $T^{\star} Q \cong T^{*} Q \times \mathbb{R}$ de $Q$, definida por

$$
\begin{gathered}
\langle(g, \dot{g}) \cdot(q, p, e),(g, \dot{g}) \cdot(q, \dot{q})\rangle=\langle(q, p, e),(q, \dot{q})\rangle \\
\text { para } g \in G, \dot{g} \in T_{g} G, q \in Q, p \in T_{q}^{*} Q, e \in \mathbb{R}, \dot{q} \in T_{q} Q,
\end{gathered}
$$

onde $\langle.,$.$\rangle denota o pareamente natural entre espaço cotangente, tanto afim como linear, e$ espaço tangente, sendo que estes dois pareamentos são relacionados por

$$
\langle(p, e), \dot{q}\rangle=\langle p, \dot{q}\rangle+e \quad \text { para } q \in Q, p \in T_{q}^{*} Q, e \in \mathbb{R}, \dot{q} \in T_{q} Q
$$

Sob o isomorfismo anterior, ela assume a forma

$$
\begin{gathered}
(g, X) \cdot(q, p, e)=\left(g \cdot q, g \cdot p, e-\left\langle p,\left(\operatorname{Ad}\left(g^{-1}\right)(X)\right)_{Q}(q)\right\rangle\right) \\
\operatorname{para}(g, X) \in G \ltimes \mathfrak{g}, q \in Q, p \in T_{q}^{*} Q, e \in \mathbb{R} .
\end{gathered}
$$

Em nível infinitesimal, estas ações induzem "representações de $\mathfrak{g} \ltimes \mathfrak{g}$ por campos fundamentais sobre $T Q$ e sobre $T^{\star} Q "$, ou seja, antihomomorfismos de álgebras de Lie

$$
\begin{aligned}
\mathfrak{g} \ltimes \mathfrak{g} & \longrightarrow \mathfrak{X}(T Q) \\
(X, \xi) & \longmapsto
\end{aligned}
$$

e

$$
\begin{aligned}
\mathfrak{g} \ltimes \mathfrak{g} & \longrightarrow \mathfrak{X}\left(T^{\star} Q\right) \\
(X, \xi) & \longmapsto(X, \xi)_{T^{\star} Q}
\end{aligned} .
$$


Explicitamente, temos

$$
\begin{gathered}
(X, \xi)_{T Q}(q, \dot{q})=\left(X_{Q}(q), X_{T Q}(q, \dot{q})+\xi_{Q}(q)\right) \\
\operatorname{para}(X, \xi) \in \mathfrak{g} \ltimes \mathfrak{g}, q \in Q, \dot{q} \in T_{q} Q,
\end{gathered}
$$

e

$$
\begin{gathered}
(X, \xi)_{T^{\star} Q}(q, p, e)=\left(X_{Q}(q), X_{T^{*} Q}(q, p),-\left\langle p, \xi_{Q}(q)\right\rangle\right) \\
\operatorname{para}(X, \xi) \in \mathfrak{g} \ltimes \mathfrak{g}, q \in Q, p \in T_{q}^{*} Q, e \in \mathbb{R},
\end{gathered}
$$

e em termos de coordenadas locais $\left(q^{i}, \dot{q}^{i}\right)$ de $T Q$ e coordenadas locais $\left(q^{i}, p_{i}, e\right)$ de $T^{\star} Q$ induzidas por coordenadas locais $q^{i}$ de $Q$, em termos das quais $X_{Q}$ e $\xi_{Q}$ são dados, respectivamente, pelas expressões locais $X_{Q}=X_{Q}^{i} \partial / \partial q^{i}$ e $\xi_{Q}=\xi_{Q}^{i} \partial / \partial q^{i}$, temos

$$
(X, \xi)_{T Q}=X_{Q}^{i} \frac{\partial}{\partial q^{i}}+\left(\dot{q}^{j} \frac{\partial X_{Q}^{i}}{\partial q^{j}}+\xi_{Q}^{i}\right) \frac{\partial}{\partial \dot{q}^{i}},
$$

e

$$
(X, \xi)_{T^{\star} Q}=X_{Q}^{i} \frac{\partial}{\partial q^{i}}-p_{j} \frac{\partial X_{Q}^{j}}{\partial q^{i}} \frac{\partial}{\partial p_{i}}-p_{i} \xi_{Q}^{i} \frac{\partial}{\partial e} .
$$

A seguir, faremos uso das ações introduzidas acima para tratar do problema de definir o conceito de uma simetria local na mecânica. Isso requer como pré-requisito estender o formalismo da mecânica para abranger sistemas não-autônomos. Afinal, se queremos efetuar transformações por elementos de um grupo de simetria $G$ que podem depender do instante $t$ em que são aplicadas, a noção de o que é autônomo e o que não é se torna "dependente do calibre", uma mera questão de convenção.

\subsubsection{Invariância local no formalismo lagrangiano}

Como mencionado acima, nas abordagens tradicionais da mecânica, um sistema descrito por uma lagrangiana $L: \mathbb{R} \times T Q \rightarrow \mathbb{R}$ é dito localmente $G$-invariante se para todo intervalo fechado $I$ de $\mathbb{R}$, o funcional ação definido por esta lagrangiana em $I$,

$$
S_{I}[\mathbf{q}]=\int_{I} d t L(t, \mathbf{q}(t), \dot{\mathbf{q}}(t)) \quad \text { para } \mathbf{q} \in C^{\infty}(I, Q)
$$

é invariante sob a ação de $C^{\infty}(I, G)$ definida pontualmente pela ação de $G$ em $Q$. Isto significa que, para todo $\mathrm{g} \in C^{\infty}(I, G)$ e todo $\mathrm{q} \in C^{\infty}(I, Q)$, devemos ter $S_{I}[\mathrm{~g} \cdot \mathrm{q}]=S_{I}[\mathrm{q}]$. Uma vez que esta relação tem que ser satisfeita para todo intervalo fechado $I$ de $\mathbb{R}$, e como lidamos apenas com funções suaves, podemos tomar intervalos arbitrariamente pequenos e 
concluir que a igualdade de todas essas integrais vale se e somente se há igualdade entre os integrandos pontualmente, ou seja, se e somente se, para todo $t \in I$, vale

$$
L(t,(\mathrm{~g} \cdot \mathrm{q})(t),(\mathrm{g} \cdot \mathrm{q})(t)))=L(t, \mathbf{q}(t), \dot{\mathrm{q}}(t)) .
$$

Pondo $\mathbf{X}(t)=\dot{\mathrm{g}}(t) \mathrm{g}^{-1}(t)$, essa condição torna-se equivalente a exigir que

$$
\begin{gathered}
L\left(t, g \cdot q, g \cdot \dot{q}+X_{Q}(g \cdot q)\right)=L(t, q, \dot{q}) \\
\text { para todo }(g, X) \in G \ltimes \mathfrak{g} \text { e todo }(t, q, \dot{q}) \in \mathbb{R} \times T Q .
\end{gathered}
$$

Chegamos assim ao resultado de que um sistema lagrangiano é localmente $G$-invariante se e somente se a lagrangiana que o descreve é invariante sob a ação de $T G$ [13].

\subsubsection{Invariância local no formalismo hamiltoniano}

Novamente, um sistema descrito por uma hamiltoniana $\mathcal{H}: \mathbb{R} \times T^{*} Q \rightarrow \mathbb{R} \times T^{*} Q \times \mathbb{R}$ é dito localmente $G$-invariante se para todo intervalo fechado $I$ de $\mathbb{R}$, o funcional ação definido por esta hamiltoniana em $I$,

$$
S_{I}[\mathbf{q}, \mathbf{p}]=\int_{I} d t(\langle\mathrm{p}(t), \dot{\mathrm{q}}(t)\rangle-H(t, \mathbf{q}(t), \mathrm{p}(t))) \quad \text { para }(\mathbf{q}, \mathbf{p}) \in C^{\infty}\left(I, T^{*} Q\right)
$$

é invariante sob a ação de $C^{\infty}(I, G)$ definida pontualmente pela ação de $G$ em $T^{*} Q$. Isto significa que, para todo $\mathrm{g} \in C^{\infty}(I, G)$ e todo $(\mathrm{q}, \mathrm{p}) \in C^{\infty}\left(I, T^{*} Q\right)$, devemos ter $S_{I}[\mathrm{~g} \cdot \mathrm{q}, \mathrm{g} \cdot \mathrm{p}]$ $=S_{I}[\mathbf{q}, \mathrm{p}]$, o que, pelo mesmo argumento dado acima, será o caso se e somente se, para todo $t \in I$, vale

$$
\langle(\mathrm{g} \cdot \mathrm{p})(t),(\mathrm{g} \cdot \mathrm{q})(t)\rangle-H(t,(\mathrm{~g} \cdot \mathrm{q})(t),(\mathrm{g} \cdot \mathrm{p})(t)))=\langle\mathrm{p}(t), \dot{\mathrm{q}}(t)\rangle-H(t, \mathrm{q}(t), \mathrm{p}(t)) .
$$

Pondo $\mathbf{X}(t)=\dot{\mathrm{g}}(t) \mathrm{g}^{-1}(t)$, essa condição torna-se equivalente a exigir que

$$
\begin{gathered}
\left\langle p,\left(\operatorname{Ad}\left(g^{-1}\right)(X)\right)_{Q}(q)\right\rangle-H((g, X) \cdot(t, q, p))=-H(t, q, p) \\
\text { para todo }(g, X) \in G \ltimes \mathfrak{g} \text { e todo }(t, q, p) \in \mathbb{R} \times T^{*} Q .
\end{gathered}
$$

Observa-se que nesta última equação, a função hamiltoniana sofre apenas a ação da parte global da simetria (ação de $G$ ), enquanto no primeiro termo entra apenas a ação da parte local da simetria (ação de $\mathfrak{g}$ ). Assim, (1.42) é satisfeita se e somente se

$$
\begin{aligned}
& H(t, g \cdot q, g \cdot p)=H(t, q, p) \text { e }\left\langle p, X_{Q}(q)\right\rangle=0 \\
& \text { para todo }(g, X) \in G \ltimes \mathfrak{g} \text { e todo }(t, q, p) \in \mathbb{R} \times T^{*} Q .
\end{aligned}
$$


Esta última equação admite uma interpretação bem interessante: lembrando que a aplicação momento para a ação de $G$ no fibrado cotangente definida pelo levantamento de uma ação de $G$ em $Q$ é dada por

$$
\langle\mathcal{J}(q, p), X\rangle=\left\langle p, X_{Q}(q)\right\rangle,
$$

concluímos que a segunda condição na equação (1.43) significa que $\mathcal{J}(q, p)=0$.

Portanto, para que um sistema hamiltoniano seja localmente $G$-invariante, duas condições devem ser satisfeitas: a função hamiltoniana $H$ deve ser invariante sob a ação de $G$ e o espaço de fase do sistema deve ser restrito ao nível 0 da aplicação momento. Juntas, estas condições equivalem a exigir que a seção hamiltoniana $\mathcal{H}$ é invariante (melhor seria dizer: equivariante) sob a ação de $T G$.

\subsection{Aplicação momento}

Na mecânica hamiltoniana, um dos conceitos mais importantes no tratamento de sistemas com simetria é o da aplicação momento. Admitindo uma pequena mudança de notação (que será revertida no final deste parágrafo), suponha que o espaço de fase do sistema seja uma variedade denotada por $P$ - que pode ser um fibrado cotangente $T^{*} Q$, como antes, ou mais geralmente uma variedade simplética, com forma simplética $\omega$, ou até uma variedade de Poisson, com tensor de Poisson $\pi$ - e suponha que $P$ seja munida de uma ação de $G$ que preserva essa sua estrutura ( $\omega$ ou $\pi$ ). Em nível infinitesimal, esta induz uma "representação de $\mathfrak{g}$ por campos fundamentais localmente hamiltonianos sobre $P$ ", ou seja, um antihomomorfismo de álgebras de $\mathrm{Lie}^{4}$

$$
\begin{aligned}
\mathfrak{g} & \longrightarrow \mathfrak{X}_{L H}(P) \\
X & \longmapsto
\end{aligned}
$$

como antes. Por outro lado, o espaço $\mathfrak{F}(P)$ das funções sobre $P$ também possui a estrutura de uma álgebra de Lie em relação ao colchete de Poisson $\{.,$.$\} , e temos um antihomomorfismo$ de álgebras de Lie

$$
\begin{aligned}
\mathfrak{F}(P) & \longrightarrow \mathfrak{X}_{L H}(P), \\
f & \longmapsto(d f)^{\sharp},
\end{aligned}
$$

onde o operador $.^{\sharp}: \Omega^{1}(P) \rightarrow \mathfrak{X}(P)$ é induzido pelo tensor de Poisson, visto como homomorfismo estrito $\pi: T^{*} P \rightarrow T P$ de fibrados vetoriais sobre $P$. Neste contexto, uma aplicação momento pode ser vista de duas maneiras: ou como uma aplicação linear

$$
\mathfrak{d}: \mathfrak{g} \longrightarrow \mathfrak{F}(P)
$$

\footnotetext{
${ }^{4} \mathfrak{X}_{L H}(P)$ denota o espaço dos campos vetoriais localmente hamiltonianos sobre $P$, i.e., dos campos vetoriais $Z$ sobre $P$ satisfazendo $L_{Z} \omega=0$ ou $L_{Z} \pi=0$, que é uma subálgebra de Lie de $\mathfrak{X}(P)$ em relação ao colchete de Lie.
} 
ou como uma função

$$
\mathcal{J}: P \longrightarrow \mathfrak{g}^{*}
$$

sendo que estas duas interpretações se relacionam por uma simples troca de argumento,

$$
\langle\mathcal{J}, X\rangle(p)=\langle\mathcal{J}(p), X\rangle \quad \text { para } X \in \mathfrak{g}, p \in P,
$$

onde se exige que a aplicação linear (1.46) seja um homomorfismo de álgebras de Lie, ou seja, que vale

$$
\{\langle\mathcal{J}, X\rangle,\langle\mathcal{J}, Y\rangle\}=\langle\mathcal{J},[X, Y]\rangle \quad \text { para } X, Y \in \mathfrak{g},
$$

e que ela torne o diagrama

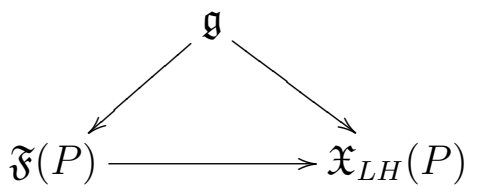

comutativo, ou seja, que vale

$$
(d\langle\mathcal{J}, X\rangle)^{\sharp}=X_{P} \quad \text { para } X \in \mathfrak{g} .
$$

Para uma variedade simplética, esta fórmula assume a seguinte forma:

$$
i_{X_{P}} \omega=d\langle\mathcal{J}, X\rangle \quad \text { para } X \in \mathfrak{g} .
$$

Para um fibrado cotangente, $P=T^{*} Q$, uma aplicação momento sempre existe e pode ser construída explicitamente usando a forma canônica $\theta$, desde que $\theta$ seja preservada pela ação de $G$ em $T^{*} Q$ - o que é o caso se (e somente se) esta ação for induzida por uma ação de $G$ em $Q$. De fato, sob estas hipóteses, vale $L_{X_{T^{*} Q}} \theta=0$ e portanto basta definir

$$
\langle\mathcal{J}, X\rangle=i_{X_{T^{*} Q}} \theta \quad \text { para } X \in \mathfrak{g},
$$

pois então

$$
d\langle\mathcal{J}, X\rangle=d i_{X_{T^{*} Q}} \theta=L_{X_{T^{*} Q}} \theta-i_{X_{T^{*} Q}} d \theta=i_{X_{T^{*} Q}} \omega .
$$

Explicitamente, a definição (1.53) pode ser reescrita usando apenas os campos fundamentais $X_{Q}$, em vez dos campos fundamentais $X_{T^{*} Q}$ :

$$
\langle\mathcal{J}, X\rangle(q, p)=\left\langle p, X_{Q}(q)\right\rangle \quad \text { para } X \in \mathfrak{g}, q \in Q, p \in T_{q}^{*} Q .
$$

A mesma construção vale, mais geralmente, para variedades simpléticas exatas onde $\omega=-d \theta$ e $\theta$ é preservada sob a ação do grupo.

Finalmente, tendo em vista que invariância global e invariância local em sistemas hamiltonianos podem ser formuladas como invariância sob a ação do grupo de Lie $G$ e do grupo 
de Lie $T G$, respectivamente, na variedade simplética exata $P=\mathbb{R} \times T^{*} Q \times \mathbb{R}$, podemos perguntar como se relacionam as respectivas aplicações momento. Primeiro, queremos investigar se a ação de $T G$ também preserva a forma canônica $\theta$ dada pela equação (1.1). Para tanto, podemos usar a expressão para o campo fundamental $(X, \xi)_{P}$ em coordenadas locais, que é dada pela equação (1.40) (não havendo termo proporcional a $\partial / \partial t$ ):

$$
(X, \xi)_{P}=X_{Q}^{i} \frac{\partial}{\partial q^{i}}-p_{j} \frac{\partial X_{Q}^{j}}{\partial q^{i}} \frac{\partial}{\partial p_{i}}-p_{i} \xi_{Q}^{i} \frac{\partial}{\partial e} .
$$

Inserindo esta expressão nas equações (1.1) e (1.2), vem

$$
i_{(X, \xi)_{P}} \theta=p_{i} X_{Q}^{i},
$$

$\mathrm{e}$

$$
i_{(X, \xi)_{P}} \omega=X_{Q}^{i} d p_{i}+p_{j} \frac{\partial X_{Q}^{j}}{\partial q^{i}} d q^{i}+p_{i} \xi_{Q}^{i} d t,
$$

implicando

$$
d i_{(X, \xi)_{P}} \theta=X_{Q}^{i} d p_{i}+p_{j} \frac{\partial X_{Q}^{j}}{\partial q^{i}} d q^{i}+p_{i} \frac{\partial X_{Q}^{i}}{\partial t} d t .
$$

Logo, usando a fórmula da derivada de Lie

$$
L_{(X, \xi)_{P}} \theta=i_{(X, \xi)_{P}} d \theta+d i_{(X, \xi)_{P}} \theta,
$$

obtemos

$$
L_{(X, \xi)_{P}} \theta=p_{i}\left(\frac{\partial X_{Q}^{i}}{\partial t}-\xi_{Q}^{i}\right) d t .
$$

Supondo que esta expressão se anula, podemos definir a aplicação momento para a ação local por

$$
\begin{gathered}
\left\langle\mathcal{J}_{\text {loc }},(X, \xi)\right\rangle(t, q, p, e)=\left\langle p, X_{Q}(q)\right\rangle \\
\operatorname{para}(X, \xi) \in \mathfrak{g} \ltimes \mathfrak{g}, q \in Q, p \in T_{q}^{*} Q, e \in \mathbb{R}
\end{gathered},
$$

e observamos que ela coincide com a aplicação momento para a ação global, que é dada por

$$
\begin{aligned}
& \left\langle\mathcal{J}_{\mathrm{glb}}, X\right\rangle(t, q, p, e)=\left\langle p, X_{Q}(q)\right\rangle \\
& \operatorname{para} X \in \mathfrak{g}, q \in Q, p \in T_{q}^{*} Q, e \in \mathbb{R}
\end{aligned}
$$

Esta é a justificativa matematicamente rigorosa para o teorema folclórico que afirma que as simetrias locais não contribuem à aplicação momento.

Permanece então a questão de entender por que o lado direito da equação (1.55) deve se anular. A rigor, o formalismo apresentado acima nem permite introduzir uma dependência temporal explícita nos campos fundamentais $X_{Q}$ ou $(X, \xi)_{P}$. Este problema pode ser superado quando passamos de ações de grupos de Lie em variedades para ações de fibrados de grupos de Lie em fibrados, o que será um dos temas centrais do próximo capítulo. 


\subsection{Redução simplética}

Uma construção que desempenha um papel central na mecânica hamiltoniana é o método de eliminação de variáveis em sistemas com simetrias, cuja versão geométrica é conhecida como redução simplética ou redução de Marsden-Weinstein. Para descrever este procedimento, suponha que $P$ é uma variedade simplética munida de uma ação de um grupo de Lie $G$ com álgebra de Lie $\mathfrak{g}$ e com aplicação momento associada $\mathcal{J}: P \rightarrow \mathfrak{g}^{*}$. Seja $\mu$ um vetor em $\mathfrak{g}^{*} \mathrm{e}$ seja

$$
G_{\mu}=\left\{g \in G / \operatorname{Ad}^{*}(g) \mu=\mu\right\}
$$

o grupo de estabilidade de $\mu$ em relação à representação coadjunta, enquanto que, para qualquer ponto $p$ de $P$,

$$
G_{p}=\{g \in G / g \cdot p=p\}
$$

denota o grupo de estabilidade de $p$ em relação à ação de $G$. Como supomos que $\mathcal{J}$ é equivariante, temos

$$
G_{p} \subset G_{\mu} \quad \text { para } p \in \mathcal{J}^{-1}(\mu) .
$$

Geralmente, considera-se a situação em que $\mu$ é um valor regular de $\mathcal{J}$ e a ação de $G_{\mu}$ sobre $\mathfrak{J}^{-1}(\mu)$ é própria e livre; veja, por exemplo, [1], Teorema 4.3.1, p. 299. No entanto, na maioria dos casos, estas hipóteses são demasiadamente restritivas, não permitindo tratar do caso que é de interesse em teorias de calibre, onde $\mu=0$. Felizmente, existe uma versão mais geral do teorema que, esta sim, é suficiente:

Teorema 1.1 (Marsden-Weinstein) Seja $\mu$ um valor quase-regular de $\mathcal{J}$ e suponha que $G_{\mu}$ aja propriamente em $\mathfrak{J}^{-1}(\mu)$ e tal que todos os grupos de estabilidade $G_{p}\left(p \in \mathcal{J}^{-1}(\mu)\right)$ sejam conjugados em $G_{\mu}$. Então $\mathcal{J}^{-1}(\mu)$ é uma subvariedade de $P$ e o quociente

$$
P_{\mu}=\mathcal{J}^{-1}(\mu) / G_{\mu}
$$

é uma variedade simplética em relação à forma simplética $\omega_{\mu}$ determinada por

$$
\pi_{\mu}^{*} \omega_{\mu}=i_{\mu}^{*} \omega
$$

onde $\pi_{\mu}$ é a projeção canônica de $\mathcal{J}^{-1}(\mu)$ sobre $P_{\mu}$ e $i_{\mu}$ é a inclusão natural de $\mathcal{J}^{-1}(\mu)$ em $P$.

Lembramos que a condição de que $\mu$ seja um valor regular de $\mathcal{J}$ significa que para qualquer ponto $p$ de $\mathcal{J}^{-1}(\mu)$, a aplicação tangente $T_{p} \mathcal{J}: T_{p} P \rightarrow \mathfrak{g}^{*}$ é sobrejetora, o que já garante que $\mathcal{J}^{-1}(\mu)$ será subvariedade de $P$, enquanto que a condição mais fraca de que $\mu$ seja um valor quase-regular de $\mathcal{J}$ significa, por definição, que $\mathcal{J}^{-1}(\mu)$ é uma subvariedade de $P$ tal que para qualquer ponto $p$ de $\mathcal{J}^{-1}(\mu)$, vale $T_{p} \mathcal{J}^{-1}(\mu)=\operatorname{ker} T_{p} \mathcal{J}$. 
O caso particular de interesse neste trabalho é quando o espaço de fase $P$ é o fibrado cotangente $T^{*} Q$ de um espaço de configuração $Q$, a ação de $G$ em $T^{*} Q$ é obtida por levantamento de uma ação de $G$ em $Q$ e $\mathcal{J}$ é a aplicação momento canônica definida pelas equações (1.53) e (1.54). Neste caso, temos

Teorema 1.2 Suponha que a ação de $G$ em $Q$ é livre e própria (isso garante que $Q / G$ é uma variedade e que a projeção $\rho: Q \rightarrow Q / G$ é uma submersão). Então a variedade simplética reduzida $\left(T^{*} Q\right)_{\alpha}$ é um fibrado sobre $T^{*}(Q / G)$ cuja fibra típica é a órbita coadjunta $\mathcal{O}_{\alpha}$

Se considerarmos o caso especial da redução do fibrado cotangente no nível 0 da aplicação momento - caso que é de grande importância para nós, pelas razões mencionadas na seção 1.1 - temos o seguinte resultado: e $\alpha$ é 0 - a situação típica para simetrias locais, como vimos acima.

Teorema 1.3 Suponha novamente que a ação de $G$ em $Q$ é livre e própria. Então 0 é um valor regular de J $\mathrm{e}$ a aplicação

$$
\left.\varphi_{0}:\left(\left(T^{*} Q\right)_{0},\left(\omega_{Q}\right)_{0}\right)\right) \rightarrow\left(T^{*}(Q / G), \omega_{Q / G}\right)
$$

dada por $\varphi_{0}\left(\left[\alpha_{q}\right]\right)\left(d_{q} \rho\left(v_{q}\right)\right)=\alpha_{q}\left(v_{q}\right)$, onde $\alpha_{q} \in \mathcal{J}^{-1}(0)$ e $v_{q} \in T_{q} Q$, é um difeomorfismo simplético 

CAPÍTUlo 2

\section{Fibrados, Jatos e Conexões}

A meta deste capítulo é apresentar alguns conceitos básicos da geometria diferencial que desempenham um papel central neste trabalho. Apesar de se tratar de material que já pode ser considerado padrão, não parece haver, até o presente, nenhuma referência contendo todos as construções pertinentes, e também existe uma grande diversidade na notação empregada na literatura, o que dificulta obter uma visão integrada do assunto.

\section{$2.1 \quad$ Fibrados}

A noção de fibrado ocupa uma posição fundamental em geometria diferencial. Existem muitos tipos diferentes de fibrados, entre os quais podemos destacar os fibrados vetoriais e os fibrados principais. Quando se quer chegar a um denominador comum entre eles, costuma-se introduzir fibrados principais e considerar todos os demais fibrados como fibrados associados - uma abordagem que se tornou padrão com o livro texto de Kobayashi-Nomizu [20]. Porém, a definição de fibrado principal carece de uma motivição mais aprofundada que no entanto existe no conceito de fibrado com grupo estrutural que já foi usado no livro clássico de Steenrod [29]. Esta tradição, quase esquecida, merece ser revitalizada.

\subsubsection{Fibrados gerais}

O conceito mais geral de fibrado pode ser formulado da seguinte forma.

Definição 2.1 Um fibrado geral ou simplesmente fibrado é uma quádrupla $(E, M, \pi, Q)$ composta de

(i) uma variedade E chamada o espaço total, 
(ii) uma variedade $M$ chamada o espaço base,

(iii) uma aplicação suave sobrejetora $\pi: E \rightarrow M$ chamada a projeção,

(iv) uma variedade $Q$ chamada a fibra típica ou a variedade modelo,

e satisfazendo o postulado de trivialidade local: existem um recobrimento aberto $\left(U_{\alpha}\right)_{\alpha \in A}$ de $M$ e uma família $\left(\Phi_{\alpha}\right)_{\alpha \in A}$ de difeomorfismos

$$
\Phi_{\alpha}: \pi^{-1}\left(U_{\alpha}\right) \longrightarrow U_{\alpha} \times Q
$$

com $\operatorname{pr}_{1} \circ \Phi_{\alpha}=\pi$ para todo $\alpha \in A$, chamados de trivializações locais. Nota-se então que para quaisquer $\alpha, \beta \in A$ com $U_{\alpha} \cap U_{\beta} \neq \emptyset$, a aplicação

$$
\Phi_{\alpha} \circ \Phi_{\beta}^{-1}:\left(U_{\alpha} \cap U_{\beta}\right) \times Q \longrightarrow\left(U_{\alpha} \cap U_{\beta}\right) \times Q
$$

é um difeomorfismo que pode também ser visto como uma função

$$
g_{\alpha \beta}: U_{\alpha} \cap U_{\beta} \longrightarrow \operatorname{Diff}(Q),
$$

chamada a correspondente função de transição, conforme a fórmula

$$
\left(\Phi_{\alpha} \circ \Phi_{\beta}^{-1}\right)(m, q)=\left(m, g_{\alpha \beta}(m)(q)\right) \quad \text { para } m \in U_{\alpha} \cap U_{\beta}, q \in Q .
$$

A seguir, especificaremos um fibrado $(E, M, \pi, Q)$ dizendo que $E$ é um fibrado sobre $M$ com projeção $\pi: E \rightarrow M$ e fibra típica $Q$. Para todo $m \in M, E_{m}=\pi^{-1}(\{m\})$ é a fibra de $E$ sobre $m$.

Note que podemos sempre escrever o espaço total de um fibrado geral como a união disjunta das suas fibras, o que deixa óbvio qual é a definição da projeção $\pi$ :

$$
E=\bigcup_{m \in M} E_{m} \quad, \quad \pi(u)=m \quad \text { para } u \in E_{m} .
$$

Esta decomposição pode ser vista como uma folheação regular de $E$ por variedades $E_{m}$ mergulhadas em $E$ como subvariedades e todas difeomorfas à variedade modelo $Q$, sendo que para cada $m \in M$, podemos escolher uma trivialização $\Phi: \pi^{-1}(U) \rightarrow U \times Q$ de $E$ sobre uma vizinhança aberta $U$ de $m$ em $M$ cuja restrição à fibra $E_{m}$ fornece um difeomorfismo

$$
\left.\Phi\right|_{E_{m}}: E_{m} \longrightarrow Q
$$

Para cálculos explícitos, é útil observar que o espaço total $E$ de um fibrado geral, visto como variedade, possui um tipo particular de cartas, construídas a partir de uma carta do espaço base $M$, uma carta da fibra típica $Q$ e uma trivialização local. Usando a notação de 
coordenadas locais, podemos formular esta construção da seguinte forma: combinando um sistema de coordenadas locais $x^{\mu}$ em $M$ e um sistema de coordenadas locais $q^{i}$ em $Q$ com uma trivialização local de $E$ sobre $M$, obtemos um sistema de coordenadas locais $\left(x^{\mu}, q^{i}\right)$ em $E$ que chamaremos de coordenadas locais adaptadas, onde "adaptadas" significa "adaptadas à estrutura de $E$ como fibrado sobre $M$, em relação à projeção $\pi$ ". Ademais, combinando uma transformação de coordenadas locais $x^{\mu} \sim x^{\prime \kappa}$ em $M$ e uma transformação de coordenadas locais $q^{i} \leadsto q^{\prime k}$ em $Q$ com uma mudança de trivialização local de $E$ sobre $M$, obtemos uma transformação de coordenadas locais adaptadas $\left(x^{\mu}, q^{i}\right) \leadsto\left(x^{\prime \kappa}, q^{\prime k}\right)$ em $E$, onde

$$
x^{\prime \kappa}=x^{\prime \kappa}\left(x^{\mu}\right) \quad \text { e } \quad q^{\prime k}=q^{\prime k}\left(x^{\mu}, q^{i}\right) .
$$

De forma geral, podemos definir a restrição de um fibrado a um subconjunto aberto qualquer do seu espaço base: dado qualquer subconjunto aberto $N$ de uma variedade $M$, um fibrado $E$ sobre $M$ com projeção $\pi$ induz, de forma natural, um fibrado $\left.E\right|_{N}$ sobre $N$ com projeção $\pi_{N}$ chamado a restrição de $E$ a $N$, definido por $\left.E\right|_{N}=\pi^{-1}(N)$ e $\pi_{N}=\left.\pi\right|_{\left.E\right|_{N}}$. (As trivializações locais de $\left.E\right|_{N}$ são obtidas das trivializações locais de $E$ por restrição.) Por outro lado, o exemplo mais simples de um fibrado sobre uma variedade $M$ é o fibrado trivial padrão definido por

$$
E=M \times Q \quad \text { e } \quad \pi=\operatorname{pr}_{1} .
$$

enquanto que para um fibrado ser chamado de trivial, basta que seja apenas isomorfo a este. Portanto, precisamos esclarecer quais são as aplicações compatíveis com a estrutura de fibrado.

Definição 2.2 Sejam $E$ e $F$ fibrados sobre $M$ e $N$ com projeções $\pi_{E}: E \rightarrow M e$ $\pi_{F}: F \rightarrow N$ e fibras típicas $Q_{E}$ e $Q_{F}$, respectivamente. Um morfismo ou homomorfismo de fibrados de $E$ em $F$ é um par $(f, \check{f})$ de aplicações suaves $f: E \rightarrow F$ e $\check{f}: M \rightarrow N$ tais que o seguinte diagrama comuta:

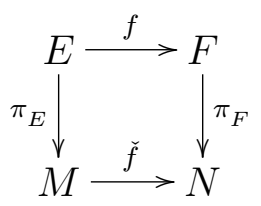

Também dizemos que $f$ é um morfismo ou homomorfismo de fibrados sobre $\check{f}$ ou que $f$ recobre $\check{f}$. Se $M=N$ e $\check{f}$ é a aplicação identidade, dizemos que $f$ é um morfismo ou homomorfismo estrito.

Notamos que esta definição simplesmente afirma que um homomorfismo de fibrados é uma aplicação suave $f$ entre os espaços totais que preserva fibras, i.e., tal que para todo $e_{1}, e_{2} \in E$ vale

$$
\pi_{E}\left(e_{1}\right)=\pi_{E}\left(e_{2}\right) \quad \Longrightarrow \quad \pi_{F}\left(f\left(e_{1}\right)\right)=\pi_{F}\left(f\left(e_{2}\right)\right) .
$$


De fato, neste caso, a aplicação $\check{f}: M \rightarrow N$ pode ser construída a partir da aplicação $f: E \rightarrow F$ pondo

$$
\check{f}\left(\pi_{E}(e)\right)=\pi_{F}(f(e)) \quad \text { para } e \in E .
$$

Também vemos que se $f: E \rightarrow F$ é um homomorfismo de fibrados sobre $\check{f}: M \rightarrow N$ e se escolhermos duas trivializações $\Phi: \pi_{E}^{-1}(U) \rightarrow U \times Q_{E}$ de $E$ e $\Psi: \pi_{F}^{-1}(V) \rightarrow V \times Q_{F}$ de $F$ sobre abertos $U$ de $M$ e $V$ de $N$, respectivamente, tais que $\check{f}(U) \subset V$ (caso contrário, substituímos o aberto $U$ pelo aberto $U \cap \check{f}^{-1}(V)$, desde que este não seja vazio), obtemos uma aplicação suave

$$
\Psi \circ f \circ \Phi^{-1}: U \times Q_{E} \longrightarrow V \times Q_{F}
$$

que pode também ser vista como uma aplicação

$$
f_{\Psi, \Phi}: U \longrightarrow C^{\infty}\left(Q_{E}, Q_{F}\right)
$$

chamada a correspondente representação local de $f$, conforme a fórmula

$$
\left(\Psi \circ f \circ \Phi^{-1}\right)(m, q)=\left(\check{f}(m), f_{\Psi, \Phi}(m)(q)\right) \quad \text { para } m \in U, q \in Q_{E} \text {. }
$$

Da noção de homomorfismo, decorrem da forma usual as de isomorfismo (= homomorfismo inversível), de endomorfismo (= homomorfismo com $E=F$ ) e de automorfismo (= isomorfismo com $E=F$ ), estritos ou não. De qualquer forma, um isomorfismo $f: E \rightarrow F$ entre fibrados sempre induz um difeomorfismo $\check{f}: M \rightarrow N$ entre as respectivas variedades base. Em particular, um fibrado $E$ sobre $M$ com fibra típica $Q$ é chamado trivial se existe um isomorfismo estrito de fibrados $f: E \rightarrow M \times Q$ e, para qualquer subconjunto aberto $N$ de $M$, é chamado trivial sobre $N$ se existe um isomorfismo estrito de fibrados $f_{N}:\left.E\right|_{N} \rightarrow N \times Q$, sendo que qualquer tal isomorfismo estrito de fibrados é chamado uma trivialização de $E$ no primeiro caso e uma trivialização de $E$ sobre $N$ ou, quando não queremos especificar $N$ explicitamente, uma trivialização local de $E$ no segundo caso.

Outro conceito de importância fundamental é o de seção:

Definição 2.3 Seja $E$ um fibrado sobre $M$ com projeção $\pi: E \rightarrow M$ e fibra típica $Q$. Uma seção de E é uma aplicação $\varphi: M \rightarrow E$ tal que $\pi \circ \varphi=\mathrm{id}_{M}$, isto é,

$$
\varphi(m) \in E_{m} \quad \text { para } m \in M
$$

Mais geralmente, uma seção de $E$ sobre um aberto $N$ de $M$ é uma seção de $\left.E\right|_{N}$. Quando não queremos especificar $N$ explicitamente, falamos de uma seção local de $E$. 


\subsubsection{Fibrados com grupo estrutural}

O maior inconveniente dos fibrados gerais reside na dificuldade de formular, com precisão matemática, a condição de que as suas funções de transição sejam suaves. De fato, as funções de transição de um fibrado geral assumem valores no grupo de difeomorfismos da fibra típica que, formalmente, pode ser visto como um grupo de Lie de dimensão infinita mas que, muitas vezes, não possui nenhuma estrutura de variedade razoável, muito menos de grupo de Lie. Por outro lado, no caso de fibrados vetoriais, por exemplo, as funções de transição tomam valores no grupo geral linear, ou no caso de fibrados vetoriais munidos de alguma estrutura algébrica adicional, em algum subgrupo fechado do grupo geral linear, e todos estes são grupos de Lie de dimensão finita. O maior inconveniente neste caso é a hipótese, demasiadamente restritiva, de que a fibra típica seja um espaço vetorial no qual o grupo pertinente age linearmente.

Estas observações sugerem, como compromisso, a seguinte definição:

Definição 2.4 Um fibrado com grupo estrutural é uma quíntupla $(E, M, \pi, Q, G)$ composta de

(i) uma variedade E chamada o espaço total,

(ii) uma variedade $M$ chamada o espaço base,

(iii) uma aplicação suave sobrejetora $\pi: E \rightarrow M$ chamada a projeção,

(iv) uma variedade $Q$ chamada a fibra típica ou a variedade modelo,

(v) um grupo de Lie $G$ chamado o grupo estrutural, em conjunto com uma ação à esquerda

$$
\begin{aligned}
G \times Q & \longrightarrow Q \\
(g, q) & \longmapsto g \cdot q
\end{aligned}
$$

na fibra típica $Q$,

e satisfazendo o postulado de trivialidade local: existem um recobrimento aberto $\left(U_{\alpha}\right)_{\alpha \in A}$ de $M$ e uma família $\left(\Phi_{\alpha}\right)_{\alpha \in A}$ de difeomorfismos

$$
\Phi_{\alpha}: \pi^{-1}\left(U_{\alpha}\right) \longrightarrow U_{\alpha} \times Q,
$$

com $\operatorname{pr}_{1} \circ \Phi_{\alpha}=\pi$ para todo $\alpha \in A$, chamados de trivializações locais, tais que para quaisquer $\alpha, \beta \in A$ com $U_{\alpha} \cap U_{\beta} \neq \emptyset$, a aplicação

$$
\Phi_{\alpha} \circ \Phi_{\beta}^{-1}:\left(U_{\alpha} \cap U_{\beta}\right) \times Q \longrightarrow\left(U_{\alpha} \cap U_{\beta}\right) \times Q
$$


é um difeomorfismo que pode ser representado por uma função suave

$$
g_{\alpha \beta}: U_{\alpha} \cap U_{\beta} \longrightarrow G,
$$

chamada a correspondente função de transição, conforme a fórmula

$$
\left(\Phi_{\alpha} \circ \Phi_{\beta}^{-1}\right)(m, q)=\left(m, g_{\alpha \beta}(m) \cdot q\right) \quad \text { para } m \in U_{\alpha} \cap U_{\beta}, q \in Q .
$$

A seguir, especificaremos um fibrado com grupo estrutural $(E, M, \pi, Q, G)$ dizendo que E é um fibrado com grupo estrutural $G$ ou simplesmente um $G$-fibrado sobre $M$ com projeção $\pi: E \rightarrow M$ e fibra típica $Q$. Para todo $m \in M, E_{m}=\pi^{-1}(\{m\})$ é a fibra de E sobre $m$.

\subsubsection{Fibrados principais}

Entre os fibrados com grupo estrutural, existe uma classe especial, os chamados fibrados principais, que podem ser caracterizados como aqueles onde $Q=G \operatorname{com} G$ agindo em si mesmo por translações à esquerda. Estes ocupam uma posição central na teoria, pois todos os outros podem ser obtidos a partir deles usando um processo chamado formação de fibrados associados.

Definição 2.5 Um fibrado principal é uma quádrupla $(P, M, \rho, G)$ que é um fibrado com fibra típica $G$ e grupo estrutural $G$ que age em si mesmo por translações à esquerda.

Para mostrar que esta definição é equivalente àquela encontrada em praticamente todos os livros texto da área, observamos que se $P$ é um fibrado principal sobre $M$ neste sentido, podemos introduzir uma ação de $G$ à direita no espaço total $P$, denotada por

$$
\begin{gathered}
P \times G \quad \longrightarrow P \\
(p, g) \\
\longmapsto p \cdot g
\end{gathered},
$$

como segue: dado um recobrimento aberto $\left(U_{\alpha}\right)_{\alpha \in A}$ de $M$ e uma família $\left(\Phi_{\alpha}\right)_{\alpha \in A}$ de trivializações locais

$$
\Phi_{\alpha}: \rho^{-1}\left(U_{\alpha}\right) \longrightarrow U_{\alpha} \times G,
$$

como na equação (2.17), definindo difeomorfismos

$$
\Phi_{\alpha} \circ \Phi_{\beta}^{-1}:\left(U_{\alpha} \cap U_{\beta}\right) \times G \longrightarrow\left(U_{\alpha} \cap U_{\beta}\right) \times G,
$$

como na equação (2.18), representados por funções de transição

$$
g_{\alpha \beta}: U_{\alpha} \cap U_{\beta} \longrightarrow G
$$


como na equação (2.19), tais que

$$
\left(\Phi_{\alpha} \circ \Phi_{\beta}^{-1}\right)(m, g)=\left(m, g_{\alpha \beta}(m) g\right) \quad \text { para } m \in U_{\alpha} \cap U_{\beta}, g \in G,
$$

como na equação (2.20), definimos a ação (2.21) pelo requerimento de que os difeomorfismos $\Phi_{\alpha}$ sejam equivariantes em relação a esta ação e a ação natural de $G$ em si mesmo por translações à direita, ou seja, que valha

$$
\Phi_{\alpha}(p)=\left(m, g_{\alpha}\right) \Longrightarrow \Phi_{\alpha}(p \cdot g)=\left(m, g_{\alpha} g\right) \quad \text { para } p \in \rho^{-1}\left(U_{\alpha}\right), g \in G \text {, }
$$

sendo que esta definição não depende da trivialização local, pois para $p \in \rho^{-1}\left(U_{\alpha} \cap U_{\beta}\right)$ e $g \in G$, a validade desta equação com $\alpha$ implica a sua validade com $\alpha$ substituído por $\beta$ :

$$
\begin{aligned}
\Phi_{\beta}(p)=\left(m, g_{\beta}\right) & \Longrightarrow \Phi_{\alpha}(p)=\left(m, g_{\alpha \beta}(m) g_{\beta}\right) \\
& \Longrightarrow \Phi_{\alpha}(p \cdot g)=\left(m,\left(g_{\alpha \beta}(m) g_{\beta}\right) g\right)=\left(m, g_{\alpha \beta}(m)\left(g_{\beta} g\right)\right) \\
& \Longrightarrow \Phi_{\beta}(p \cdot g)=\left(m, g_{\beta} g\right) .
\end{aligned}
$$

Agora é óbvio que a ação de $G$ em $P$ à direita assim definida preserva as fibras de $P$ e age livre e transitivamente nas fibras de $P$, dando origem à definição usual de um fibrado principal, onde a existência desta ação costuma ser postulada sem nenhum motivo aparente. Porém, a construção que acabamos de apresentar revela a idéia que está atrás deste postulado, pois é a existência desta ação, em conjunto com o postulado da existência de trivializações locais equivariantes, que restringe as funções de transição de tal maneira a tomarem valores num subgrupo de dimensão finita do grupo de difeomorfismos da fibra típica $G$ : Os difeomorfismos de um grupo de Lie $G$ que comutam com a ação de $G$ em si mesmo por translações à direita são justamente aqueles obtidos pela ação de $G$ em si mesmo por translações à esquerda.

Para uso posterior, notamos aqui que, por construção, as trivializações utilizadas acima são equivariantes em relação à ação (2.21). Reciprocamente, se considerarmos a ação (2.21) como dada "a priori", a definição de fibrado principal exige a existência de uma família de trivializações locais equivariantes cujos domínios recobrem a variedade base. De forma geral, uma trivialização $\Phi: \rho^{-1}(U) \rightarrow U \times G$ de $P$ sobre $U$ é equivariante se, quando escrevemos $\Phi$ na forma

$$
\Phi(p)=\left(\rho(p), \Phi_{2}(p)\right) \quad \text { para } p \in \rho^{-1}(U),
$$

$\operatorname{com} \Phi_{2} \in C^{\infty}\left(\rho^{-1}(U), G\right)$, vale

$$
\Phi(p \cdot g)=\left(\rho(p), \Phi_{2}(p) g\right) \quad \text { para } p \in \rho^{-1}(U), g \in G .
$$

Ela pode então ser definida inteiramente em termos de uma seção $\sigma$ de $P$ sobre $U$, obtida por $\sigma(m)=\Phi^{-1}(m, 1)$ ou $\Phi_{2}(\sigma(m))=1$, pois esta determina o seu inverso através da fórmula

$$
\Phi^{-1}(m, g)=\sigma(m) \cdot g \quad \text { para } m \in U, g \in G \text {. }
$$


Em termos destas seções, a definição (2.25) das funções de transição assume a forma

$$
\sigma_{\beta}(m)=\sigma_{\alpha}(m) \cdot g_{\alpha \beta}(m) \quad \text { para } m \in U_{\alpha} \cap U_{\beta},
$$

pois esta relação implica

$$
\begin{gathered}
\left(\Phi_{\alpha} \circ \Phi_{\beta}^{-1}\right)(m, 1)=\Phi_{\alpha}\left(\sigma_{\beta}(m)\right)=\Phi_{\alpha}\left(\sigma_{\alpha}(m) \cdot g_{\alpha \beta}(m)\right)=\left(m, g_{\alpha \beta}(m)\right) \\
\text { para } m \in U_{\alpha} \cap U_{\beta} .
\end{gathered}
$$

\subsubsection{Fibrados associados}

Um dos motivos para o nome "fibrado principal" é o fato de que qualquer fibrado com grupo estrutural $G$ pode ser construído a partir de um fibrado principal com grupo estrutural $G$, como fibrado associado. A vantagem essencial desta construção é que ela é manifestamente independente de trivializações.

Para descrever a construção, seguimos [15, Vol. II, Cap. 5]. Suponha que $(P, M, \rho, G)$ é um fibrado principal e $Q$ é uma variedade munida de uma ação à esquerda de $G$ como na equação (2.16). Consideramos então a variedade produto $P \times Q$ munida da ação à direita de $G$ composta

$$
\begin{array}{ccc}
(P \times Q) \times G & \longrightarrow & P \times Q \\
((p, q), g) & \longmapsto & \left(p \cdot g, g^{-1} \cdot q\right)
\end{array},
$$

que é uma ação livre, já que a ação de $G$ em $P$ é livre. Portanto, o espaço quociente das órbitas desta ação é uma variedade que será denotada por $P \times{ }_{G} Q$, e a classe de equivalência, ou órbita, de um ponto $(p, q)$ será denotada por $[p, q]$. Portanto, temos

$$
\left[p \cdot g, g^{-1} \cdot q\right]=[p, q] \quad \text { ou } \quad[p \cdot g, q]=[p, g \cdot q] \quad \text { para } p \in P, q \in Q, g \in G \text {. }
$$

Obviamente, temos duas projeções: a projeção canônica $\rho_{Q}$ de $P \times Q$ sobre $P \times{ }_{G} Q$ definida por

$$
\rho_{Q}(p, q)=[p, q] \quad \text { para } p \in P, q \in Q
$$

e a projeção $\pi$ de $P \times{ }_{G} Q$ sobre $M$ definida por

$$
\pi[p, q]=\rho(p) \quad \text { para } p \in P, q \in Q,
$$

que servem para completar o seguinte diagrama comutativo:

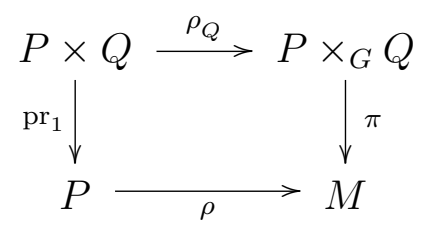


A utilidade deste diagrama reside no fato que as flechas verticais constituem fibrados com grupo estrutural $G$ e fibra típica $Q$, enquanto que as flechas horizontais definem fibrados principais com grupo estrutural $G$ :

Teorema 2.1 Com a notação introduzida acima, vale

1. $\left(P \times Q, \operatorname{pr}_{1}, P\right)$ e $\left(P \times_{G} Q, \pi, M\right)$ são fibrados com grupo estrutural $G$ e fibra típica $Q$,

2. $\left(P \times Q, \rho_{Q}, P \times{ }_{G} Q\right)$ e $(P, \rho, M)$ são fibrados principais com grupo estrutural $G$,

3. Se $U$ é um aberto de $M$, toda trivialização equivariante $\Phi_{\sigma}^{P}: \rho^{-1}(U) \rightarrow U \times G$ de $P$ sobre $U$, definida por uma seção $\sigma: U \rightarrow P$ de $P$ sobre $U$ conforme a equação (2.29), induz naturalmente uma trivialização $\Phi_{\sigma}^{Q}: \pi^{-1}(U) \rightarrow U \times Q$ de $P \times_{G} Q$ sobre $U$ tal que as funções de transição de ambas são idênticas, e também induz naturalmente uma trivialização $\tilde{\Phi}_{\sigma}^{Q}: \rho_{Q}^{-1}\left(\pi^{-1}(U)\right) \rightarrow \pi^{-1}(U) \times G$ de $P \times Q$ sobre $\pi^{-1}(U)$ tal que as funções de transição de ambas são relacionadas pela projeção $\pi$ :

$$
\begin{array}{r}
\sigma_{\beta}(m)=\sigma_{\alpha}(m) \cdot g_{\alpha \beta}(m) \quad \Longleftrightarrow\left(\Phi_{\sigma_{\alpha}}^{P} \circ\left(\Phi_{\sigma_{\beta}}^{P}\right)^{-1}\right)(m, g)=\left(m, g_{\alpha \beta}(m) g\right) \\
\Longrightarrow\left\{\begin{array}{c}
\left(\Phi_{\sigma_{\alpha}}^{Q} \circ\left(\Phi_{\sigma_{\beta}}^{Q}\right)^{-1}\right)(m, q)=\left(m, g_{\alpha \beta}(m) \cdot q\right) \\
\left(\tilde{\Phi}_{\sigma_{\alpha}}^{Q} \circ\left(\tilde{\Phi}_{\sigma_{\beta}}^{Q}\right)^{-1}\right)([p, q], g)=\left([p, q], g_{\alpha \beta}(\pi[p, q]) g\right)
\end{array}\right.
\end{array}
$$

Demonstração: Suponha que $\Phi_{\sigma}^{P}: \rho^{-1}(U) \rightarrow U \times G$ é uma trivialização equivariante de $P$ sobre $U$, definida por uma seção $\sigma: U \rightarrow P$ de $P$ sobre $U$ conforme a equação (2.29) e com segundo componente $\left(\Phi_{\sigma}^{P}\right)_{2}$ como na equação (2.27). Então definimos a aplicação $\Phi_{\sigma}^{Q}: \pi^{-1}(U) \rightarrow U \times Q$ por

$$
\Phi_{\sigma}^{Q}[p, q]=\left(\rho(p),\left(\Phi_{\sigma}^{P}\right)_{2}(p) \cdot q\right) \quad \text { para } p \in \rho^{-1}(U), q \in Q .
$$

Esta aplicação é bem definida, pois

$$
\left(\rho(p \cdot g),\left(\Phi_{\sigma}^{P}\right)_{2}(p \cdot g) \cdot\left(g^{-1} \cdot q\right)\right)=\left(\rho(p),\left(\left(\Phi_{\sigma}^{P}\right)_{2}(p) g\right) \cdot\left(g^{-1} \cdot q\right)\right)=\left(\rho(p),\left(\Phi_{\sigma}^{P}\right)_{2}(p) \cdot q\right),
$$

e é bijetora, com inversa dada por

$$
\left(\Phi_{\sigma}^{Q}\right)^{-1}(m, q)=[\sigma(m), q] \quad \text { para } m \in U, q \in Q .
$$

De forma semelhante, definimos a aplicação $\tilde{\Phi}_{\sigma}^{Q}: \rho^{-1}(U) \times Q \rightarrow \pi^{-1}(U) \times G$ por

$$
\tilde{\Phi}_{\sigma}^{Q}(p, q)=\left([p, q],\left(\Phi_{\sigma}^{P}\right)_{2}(p)\right) \quad \text { para } p \in \rho^{-1}(U), q \in Q .
$$

Esta aplicação é equivariante, pois

$$
\tilde{\Phi}_{\sigma}^{Q}((p, q) \cdot g)=\tilde{\Phi}_{\sigma}^{Q}\left(p \cdot g, g^{-1} \cdot q\right)=\left(\left[p \cdot g, g^{-1} \cdot q\right],\left(\Phi_{\sigma}^{P}\right)_{2}(p \cdot g)\right)=\left([p, q],\left(\Phi_{\sigma}^{P}\right)_{2}(p) g\right)
$$


e é bijetora, com inversa dada por

$$
\left(\tilde{\Phi}_{\sigma}^{Q}\right)^{-1}([p, q], g)=\left(p \cdot\left(\left(\Phi_{\sigma}^{P}\right)_{2}(p)^{-1} g\right),\left(g^{-1}\left(\Phi_{\sigma}^{P}\right)_{2}(p)\right) \cdot q\right) \quad \text { para } p \in \rho^{-1}(U), q \in Q, g \in G .
$$

Portanto, $\tilde{\Phi}_{\sigma}^{Q}$ é uma trivialização equivariante de $P \times Q$ sobre $\pi^{-1}(U)$, definida por uma seção $\quad \tilde{\sigma}: \pi^{-1}(U) \rightarrow P \times Q$ de $P \times Q$ sobre $\pi^{-1}(U)$ conforme a equação (2.29) e com segundo componente $\left(\tilde{\Phi}_{\sigma}^{Q}\right)_{2}$ definido como na equação $(2.27)$, sendo que

$$
\left(\tilde{\Phi}_{\sigma}^{Q}\right)_{2}(p, q)=\left(\Phi_{\sigma}^{P}\right)_{2}(p) \quad \text { para } p \in \rho^{-1}(U), q \in Q
$$

e

$$
\tilde{\sigma}([p, q])=\left(\sigma(\rho(p)) \cdot\left(\Phi_{\sigma}^{P}\right)_{2}(p)^{-1},\left(\Phi_{\sigma}^{P}\right)_{2}(p) \cdot q\right) \quad \text { para } p \in \rho^{-1}(U), q \in Q,
$$

de modo que, em particular,

$$
\tilde{\sigma}([\sigma(m), q])=(\sigma(m), q) \quad \text { para } m \in U, q \in Q .
$$

Portanto, considerando duas seções $\sigma_{\alpha}$ de $P$ sobre $U_{\alpha}$ e $\sigma_{\beta}$ de $P$ sobre $U_{\beta}$ com função de transição $g_{\alpha \beta}$ definida conforme a equação (2.30), concluímos que para $m \in U_{\alpha} \cap U_{\beta}$ e $q \in Q$, vale

$$
\begin{aligned}
\left(\Phi_{\sigma_{\alpha}}^{Q} \circ\left(\Phi_{\sigma_{\beta}}^{Q}\right)^{-1}\right)(m, q) & =\Phi_{\sigma_{\alpha}}^{Q}\left[\sigma_{\beta}(m), q\right]=\Phi_{\sigma_{\alpha}}^{Q}\left[\sigma_{\alpha}(m) \cdot g_{\alpha \beta}(m), q\right] \\
& =\Phi_{\sigma_{\alpha}}^{Q}\left[\sigma_{\alpha}(m), g_{\alpha \beta}(m) \cdot q\right]=\left(m, g_{\alpha \beta}(m) \cdot q\right),
\end{aligned}
$$

e

$$
\begin{aligned}
\tilde{\sigma}_{\beta}\left(\left[\sigma_{\alpha}(m), q\right]\right) & =\tilde{\sigma}_{\beta}\left(\left[\sigma_{\beta}(m) \cdot g_{\alpha \beta}(m)^{-1}, q\right]\right)=\tilde{\sigma}_{\beta}\left(\left[\sigma_{\beta}(m), g_{\alpha \beta}(m)^{-1} \cdot q\right]\right) \\
& =\left(\sigma_{\alpha}(m) \cdot g_{\alpha \beta}(m), g_{\alpha \beta}(m)^{-1} \cdot q\right)=\left(\sigma_{\alpha}(m), q\right) \cdot g_{\alpha \beta}(m) \\
& =\tilde{\sigma}_{\alpha}\left(\left[\sigma_{\alpha}(m), q\right]\right) \cdot g_{\alpha \beta}(m),
\end{aligned}
$$

ou seja

$$
\left(\Phi_{\sigma_{\alpha}}^{Q} \circ\left(\Phi_{\sigma_{\beta}}^{Q}\right)^{-1}\right)(m, q)=\left(m, g_{\alpha \beta}(m) \cdot q\right) \quad \text { para } m \in U_{\alpha} \cap U_{\beta}, q \in Q,
$$

$\mathrm{e}$

$$
\tilde{\sigma}_{\beta}([p, q])=\tilde{\sigma}_{\alpha}([p, q]) \cdot g_{\alpha \beta}(\pi[p, q]) \quad \text { para }[p, q] \in \pi^{-1}\left(U_{\alpha} \cap U_{\beta}\right) .
$$

Finalmente, compondo as trivializações locais $\Phi_{\sigma}^{Q}$ com cartas locais de $M$ (diminuindo os respectivos domínios se for necessário) e com cartas locais de $Q$, podemos definir cartas locais de $P \times_{G} Q$ que permitem verificar, de maneira independente, que $P \times_{G} Q$ é de fato uma variedade e $\pi$ e $\rho_{Q}$ são submersões sobrejetoras, sendo representadas, nestas cartas locais, pela projeção sobre o primeiro fator de um produto cartesiano. 
A seguir, omitiremos os índices $P$ e $Q$ nas trivializações locais $\Phi_{\sigma}^{P}$ de $P, \Phi_{\sigma}^{Q}$ de $P \times_{G} Q$ e $\tilde{\Phi}_{\sigma}^{Q}$ de $P \times Q$.

Definição 2.6 $O$ G-fibrado $P \times{ }_{G} Q$ sobre $M$ com fibra típica $Q$ é chamado o fibrado associado ao $G$-fibrado principal $P$ sobre $M$, em relação à ação (2.16).

Exemplo 2.1 Considere a situação em que a fibra típica $Q$ é o próprio grupo estrutural $G$. Existem duas ações naturais de $G$ em si mesmo que podem ser usadas para definir fibrados associados. A primeira é a ação por translações à esquerda, definida por

$$
g \cdot g^{\prime}=g g^{\prime} \quad \text { para } g, g^{\prime} \in G,
$$

enquanto que a segunda é a ação por conjugação, definida por

$$
g \cdot g^{\prime}=g g^{\prime} g^{-1} \quad \text { para } g, g^{\prime} \in G .
$$

Observamos que as duas ações são bastante diferentes entre si e portanto o mesmo vale para os correspondentes fibrados associados, que não devem ser confundidos. A seguir, denotaremos apenas o segundo pelo símbolo $P \times_{G} G$, pois o primeiro é canonicamente isomorfo ao próprio fibrado principal $P$, sendo que se a ação de $G$ em si mesmo for por translações à esquerda, podemos identificar a classe $\left[p, g^{\prime}\right]$ do $\operatorname{par}\left(p, g^{\prime}\right)$ com o ponto $p \cdot g^{\prime}$.

\subsubsection{Estruturas localmente constantes nas fibras}

$\mathrm{Na}$ grande maioria dos casos, os fibrados que aparecem em geometria diferencial e em suas aplicações a problemas de física vêm munidos de alguma estrutura adicional nas suas fibras: em vez de serem simplesmente cópias de alguma variedade, as fibras são espaços vetoriais, reais ou complexos, ou são espaços vetoriais com alguma estrutura adicional tal como um produto escalar, ou são variedades riemannianas, etc. A única restrição que deve ser imposta em todos estes casos é o requerimento da existência de trivializações locais do fibrado que sejam compatíveis com esta estrutura adicional, de modo a torná-la "localmente constante".

Mais especificamente, seja $E$ um fibrado sobre uma variedade base $M$ com projeção $\pi: E \rightarrow M$ e fibra típica $Q$, e suponha que tanto a fibra típica $Q$ como cada uma das fibras $E_{m}$ de $E$ seja munida de uma determinada estrutura geométrica do mesmo tipo. Diremos que esta estrutura geométrica é localmente constante se existe uma família $\left(\Phi_{\alpha}\right)_{\alpha \in A}$ de trivializações locais $\Phi_{\alpha}: \pi^{-1}\left(U_{\alpha}\right) \rightarrow U_{\alpha} \times Q$ de $E$ cujos domínios $U_{\alpha}$ recobrem $M$ e tal que para todo ponto $m$ de $U_{\alpha}$, o difeomorfismo $\left(\Phi_{\alpha}\right)_{m}: E_{m} \rightarrow Q$ preserva a referida estrutura: qualquer trivialização (local) deste tipo será então chamada de trivialização (local) compatível. A seguir, queremos concretizar esta idéia através de alguns exemplos importantes. 
Exemplo 2.2 Suponha que a fibra típica e cada uma das fibras $E_{m}$ de $E$ é um espaço vetorial (real ou complexo). Então a condição de que esta estrutura linear seja localmente constante significa que $E$ é um fibrado vetorial (real ou complexo) sobre $M$.

Exemplo 2.3 Continuando o exemplo anterior, suponha além disso que a fibra típica e cada uma das fibras $E_{m}$ de $E$ seja munida de um tensor ou conjunto finito de tensores, que podemos interpretar como uma seção $\sigma$ ou um conjunto finito $\Sigma=\left\{\sigma_{1}, \ldots, \sigma_{r}\right\}$ de seções de certas potências tensoriais de $E$ e seu dual $E^{*}$, descrevendo uma estrutura adicional nas fibras. Então a condição de que esta estrutura adicional também seja localmente constante significa que $E$ é um $\Sigma$-fibrado sobre $M$. Exemplos incluem fibrados riemannianos ou hermiteanos, fibrados simpléticos, fibrados complexos como fibrados reais com estrutura adicional e o conceito de um fibrado de álgebras de Lie, que desempenha um papel importante neste trabalho. Para maiores detalhes, veja [15, Vol. II, Cap. 5].

A mesma idéia prevalece na definição de estruturas não-lineares. Por exemplo, suponha que a fibra típica e cada uma das fibras $E_{m}$ de $E$ é um espaço afim, ou um grupo de Lie, ou uma variedade riemanniana. Então a exigência de que este tipo de estrutura seja localmente constante no sentido de que existam trivializações locais $\Phi: \pi^{-1}(U) \rightarrow U \times Q$ em torno de cada ponto da variedade base que são compatíveis no sentido de que para todo ponto $m$ de $U$, o difeomorfismo $\Phi_{m}: E_{m} \rightarrow Q$ é, respectivamente, um isomorfismo afim, ou um isomorfismo de grupos de Lie, ou uma isometria, leva à definição dos conceitos de um fibrado afim, ou de um fibrado de grupos de Lie, ou de um fibrado de variedades riemannianas, sendo que os primeiros dois tipos também desempenharão um papel importante neste trabalho.

Uma outra maneira de abordar a questão, que permite até desenvolver um arcabouço geral para o conceito de "estrutura geométrica", é baseada na codificação deste conceito em termos do grupo das transformações que a preservam, implementando assim o programa de Erlangen de Felix Klein no âmbito de fibrados. Novamente, isso nos leva a considerar fibrados com grupo estrutural, ou seja, fibrados associados a algum fibrado principal.

Mais especificamente, seja $P$ um fibrado principal sobre uma variedade base $M$ com projeção $\rho: P \rightarrow M$ e grupo estrutural $G$, e seja $Q$ uma variedade munida de uma ação à esquerda de $G$ como na equação (2.16). Nestas hipóteses, pode-se afirmar que se a variedade $Q$ vem munida de alguma estrutura adicional que é invariante sob a ação de $G$, então todas as fibras de $P \times{ }_{G} Q$ serão naturalmente munidas de uma estrutura do mesmo tipo e esta estrutura nas fibras será localmente constante.

Para concretizar esta afirmação, retornemos à série de exemplos já considerados acima.

Exemplo 2.4 Suponha que a fibra típica $Q$ é um espaço vetorial (real ou complexo) e que a ação de $\Gamma$ em $Q$ se dá por transformações lineares. Então o fibrado associado $P \times_{\Gamma} Q$ é um fibrado vetorial. 
Exemplo 2.5 Continuando o exemplo anterior, suponha além disso que a fibra típica $Q$ é munida de um tensor ou conjunto finito de tensores e que a ação de $\Gamma$ em $Q$ se dá por transformações lineares que preservam este tensor ou conjunto finito de tensores. Então o fibrado associado $P \times_{\Gamma} Q$ é um $\Sigma$-fibrado.

A mesma idéia prevalece na definição de estruturas não-lineares. Por exemplo, suponha que a fibra típica é um espaço afim, ou um grupo de Lie, ou uma variedade riemanniana, e que a ação de $\Gamma$ em $Q$ se dá, respectivamente, por transformações afins, por automorfismos ou por isometrias. Então o fibrado associado $P \times_{\Gamma} Q$ é, respectivamente, um fibrado afim, ou um fibrado de grupos de Lie, ou um fibrado de variedades riemannianas.

Reciprocamente, para estruturas geométricas cujos grupos de automorfismos são grupos de Lie (i.e., de dimensão finita), podemos a partir de um fibrado munido de uma estrutura deste tipo e localmente constante nas fibras reconstruir um fibrado principal como "fibrado de referenciais compatíveis" ao qual ele é associado. Este tipo de estrutura geométrica inclui todos os tipos de estruturas lineares e não-lineares mencionadas acima, mas não inclui, por exemplo. estruturas simpléticas ou de volume nas fibras quando estas são variedades e não espaços vetoriais.

\subsection{Jatos}

A partir de um fibrado geral $E$ sobre uma variedade base $M$, podemos obter vários outros fibrados. As construções de alguns destes serão discutidas nesta seção, entre eles o fibrado vertical, o fibrado dos jatos de primeira ordem e, por iteração, o fibrado dos jatos de segunda ordem.

\subsubsection{Fibrado vertical}

O fibrado vertical $V E$ de $E$ é o subfibrado vetorial do fibrado tangente $T E$ do espaço total $E$ cuja fibra em um ponto $e \in E$ situado sobre o ponto $m \in M$ consiste no espaço tangente à fibra de $E$ sobre $m$ no ponto $e$ :

$$
V_{e} E=T_{e}\left(E_{m}\right) .
$$

Outra maneira de defini-lo é como núcleo da aplicação tangente $T \pi: T E \rightarrow T M$ à projeção $\pi: E \rightarrow M$, i.e., $V E=\operatorname{ker}(T \pi)$, ou de forma mais explícita,

$$
V_{e} E=\left\{u_{e} \in T_{e} E / T_{e} \pi\left(u_{e}\right)=0\right\} .
$$




\subsubsection{Fibrados de jatos}

Nesta subseção, queremos rever a definição do fibrado de jatos (de ordem $k$ ) de $E$, que denotaremos por $J^{k} E$. Dado um ponto $m$ de $M$, consideramos seções locais $\varphi$ de $E$, isto é, seções da restrição de $E$ a alguma vizinhança aberta $U$ de $m$ em $M$, e introduzimos a seguinte relação de equivalência entre duas tais seções $\varphi_{1}$ e $\varphi_{2}$ (que sempre podemos imaginar como sendo definidas sobre a mesma vizinhança $U=U_{1} \cap U_{2}$ de $m$ ):

$$
\varphi_{1} \sim_{k, m} \varphi_{2} \Longleftrightarrow \begin{gathered}
\varphi_{1} \text { e } \varphi_{2} \text { coincidem até a } k \text {-ésima ordem } \\
\text { de suas expansões de Taylor em } m
\end{gathered}
$$

Chamamos as classes de equivalência determinadas por esta relação de $k$-jatos de seções (locais) de $E$ em $m$ e denotamos o $k$-jato de uma seção local $\varphi$ de $E$ em $m$ por $j_{m}^{k} \varphi$, o conjunto de todos os $k$-jatos de seções (locais) de $E$ por $J^{k} E$, o conjunto de todos os $k$-jatos de seções (locais) de $E$ em $m$ por $J_{m}^{k} E$ e o conjunto de todos os $k$-jatos de seções (locais) $\varphi$ de $E$ em $m$ tais que $\varphi(m)=e$, onde $e$ é um ponto dado de $E$, por $J_{e}^{k} E$. Existem duas projeções naturais do conjunto total $J^{k} E$ : a projeção fonte ("source projection") $\sigma^{k}: J^{k} E \rightarrow M$ dada por $\sigma^{k}\left(j_{m}^{k} \varphi\right)=m$ e a projeção alvo ("target projection") $\tau^{k}: J^{k} E \rightarrow E$ dada por $\tau^{k}\left(j_{m}^{k} \varphi\right)=\varphi(m)$. Esta última admite uma generalização natural para uma projeção $\tau_{l}^{k}: J^{k} E \rightarrow J^{l} E$, para $0 \leqslant l \leqslant k$. Usando as expressões de expansões de Taylor em coordenadas locais de $M$ e coordenadas locais adaptadas de $E$, é possível mostrar que $J^{k} E$ é uma variedade e é espaço total de um fibrado, em relação a qualquer uma das projeções mencionadas $\left(\sigma^{k}, \tau^{k}\right.$ e $\left.\tau_{l}^{k}\right)$. Em particular, segue que qualquer seção suave $\varphi$ de $E$ sobre $M$ induz uma seção suave $j^{k} \varphi$ de $J^{k} E$ sobre $M$, cujo valor no ponto $m$ de $M$ é $j_{m}^{k} \varphi$.

De forma explícita, a relação de equivalência empregada na definição (2.43) acima pode ser descrita como segue. Primeiro, denotando por $\sim_{k, e}$ a relação de equivalência definida por

$$
\varphi_{1} \sim_{k, e} \varphi_{2} \quad \text { se } \quad j_{m}^{k} \varphi_{1}=j_{m}^{k} \varphi_{2} \quad \text { e } \quad \tau^{k}\left(j_{m}^{k} \varphi_{1}\right)=e=\tau^{k}\left(j_{m}^{k} \varphi_{2}\right)
$$

temos que

$$
\varphi_{1} \sim_{k, m} \varphi_{2} \Longleftrightarrow \varphi_{1} \sim_{k, e} \varphi_{2} \quad \text { para algum } e \in E_{m} .
$$

Segundo, denotando a fibra típica de $E$ por $Q$ e fixando uma vizinhança aberta $U$ do ponto $m$ de $M$ (que será diminuída cada vez que isso se tornar necessário), escolhemos uma trivialização de $E$ sobre $U$ (i.e., um isomorfismo de fibrados $\left.E\right|_{U} \cong U \times Q$ ) para representar seções $\varphi \in \Gamma\left(\left.E\right|_{U}\right)$ de $E$ sobre $U$ por aplicações $f \in C^{\infty}(U, Q)$ de acordo com a fórmula $\varphi=\left(\operatorname{id}_{U}, f\right)$. Em seguida, escrevendo $e=(m, n)$, escolhemos um sistema de coordenadas locais $x^{\mu}$ de $M$ em torno do ponto $m$ com domínio $U$, assim como um sistema de coordenadas locais $q^{i}$ de $Q$ em torno do ponto $n$ (i.e., $x: U \rightarrow U_{0}$ é uma carta de $M$, onde $U_{0}$ é uma bola aberta em $\mathbb{R}^{n}$ em torno da origem e $x(m)=0 ; q: V \rightarrow V_{0}$, por sua vez, é uma carta de $Q$, sendo que $V$ é 
uma vizinhança aberta de $n, V_{0}$ é uma bola aberta em $\mathbb{R}^{N}$ em torno da origem e $\left.q(n)=0\right)$. Então temos

$$
\begin{gathered}
f_{1}(m)=n=f_{2}(m) \\
\varphi_{1} \sim_{k, e} \varphi_{2} \Longleftrightarrow \quad \begin{array}{c}
\mathrm{e} \\
D_{0}^{j}\left(q \circ f_{1} \circ x^{-1}\right)=D_{0}^{j}\left(q \circ f_{2} \circ x^{-1}\right) \quad \text { para } 1 \leqslant j \leqslant k
\end{array},
\end{gathered}
$$

onde $D_{0}^{j} h \in L_{s}^{j}\left(\mathbb{R}^{n}, \mathbb{R}^{N}\right)$ denota a $j$-ésima derivada em 0 , no sentido do cálculo avançado, de uma aplicação $h: U_{0} \subset \mathbb{R}^{n} \rightarrow \mathbb{R}^{N}$.

Usando a regra da cadeia para derivadas superiores, pode-se mostrar que esta definição independe da escolha da trivialização ou das coordenadas utilizadas.

Como caso particular, consideramos a situação em que o fibrado $E$ é trivial, ou seja, $E=M \times Q$ onde $Q$ é uma variedade fixa. Neste caso, costuma-se escrever $J^{k}(M, Q)$ em vez de $J^{k} E, J_{m}^{k}(M, Q)$ em vez de $J_{m}^{k} E$ e $J_{m}^{k}(M, Q)_{q}$ em vez de $J_{(m, q)}^{k} E$. Ademais, usando a identificação de seções $\varphi$ de $M \times Q$ com aplicações $f: M \rightarrow Q$ dada por $\varphi=\left(\operatorname{id}_{M}, f\right)$, costuma-se escrever os elementos de $J_{m}^{k}(M, Q)$ na forma $j_{m}^{k} f$, onde $f$ é uma aplicação de alguma vizinhança aberta $U$ de $m$ em $M$ para $Q$. Globalmente, qualquer aplicação suave $f: M \rightarrow Q$ induz uma aplicação suave $j^{k} f: M \rightarrow J^{k}(M, Q)$, cujo valor no ponto $m$ de $M$ é $j_{m}^{k} f$. Finalmente, costuma-se redefinir a projeção alvo como projeção sobre $Q$, em vez de $M \times Q$, ou seja, $\tau^{k}: J^{k}(M, Q) \rightarrow Q$ é dada por $\tau^{k}\left(j_{m}^{k} f\right)=f(m)$.

Um caso especial interessante ocorre se escolhemos $M=\mathbb{R}^{n}$ e fixamos o ponto $m$ de $M$ como sendo a origem de $\mathbb{R}^{n}$, pois $J_{0}^{k}\left(\mathbb{R}^{n}, Q\right.$ ) ainda é um fibrado (e de fato, um fibrado vetorial) sobre $Q$ em relação à projeção alvo:

$$
J_{0}^{k}\left(\mathbb{R}^{n}, Q\right)=\bigcup_{q \in Q} J_{0}^{k}\left(\mathbb{R}^{n}, Q\right)_{q}
$$

Note que para $k=1$,

$$
J_{0}^{1}(\mathbb{R}, Q)=T Q \quad, \quad J_{0}^{1}\left(\mathbb{R}^{n}, Q\right)=L\left(Q \times \mathbb{R}^{n}, T Q\right)=\left(Q \times\left(\mathbb{R}^{n}\right)^{*}\right) \otimes T Q,
$$

enquanto que pondo $n=1$ mas admitindo $k>1$, chegamos ao conceito de fibrados tangentes de ordem superior [2].

Voltando ao caso de um fibrado geral $E$, notamos que uma trivialização

$$
\Phi:\left.E\right|_{U} \longrightarrow U \times Q
$$

de $E$ sobre $U$ em conjunto com uma carta

$$
x: U \longrightarrow U_{0}
$$

\footnotetext{
${ }^{1}$ Dados dois espaços vetoriais $V$ e $W$, denotamos por $L_{s}^{k}(V, W)$ o espaço vetorial das aplicações $k$ multilineares simétricas de $V \times \ldots \times V$ ( $k$ fatores) para $W$.
} 
de $U$, onde $U_{0}$ é uma vizinhança aberta de $0 \mathrm{em} \mathbb{R}^{n}$, induz uma trivialização

$$
\begin{aligned}
& J^{k} \Phi^{x}:\left.\quad J^{k} E\right|_{U} \quad \longrightarrow \quad U \times J_{0}^{k}\left(\mathbb{R}^{n}, Q\right) \\
& j_{m}^{k} \varphi \longmapsto\left(m, j_{0}^{k}\left(\operatorname{pr}_{2} \circ \Phi \circ \varphi \circ x^{-1} \circ \tau_{x^{-1}(m)}\right)\right)
\end{aligned}
$$

de $J^{k} E$, como fibrado sobre $M$ (i.e., em relação à projeção fonte), sobre $U$. (Aqui, denotamos por $\tau_{a}: \mathbb{R}^{n} \rightarrow \mathbb{R}^{n}$ a translação pelo vetor $a \in \mathbb{R}^{n}$ que leva $u \mathrm{em} u+a$.) Em particular, quando o fibrado original é um fibrado associado a um fibrado principal $P$ com grupo estrutural $G$, $E=P \times_{G} Q$, e quando as suas trivializações locais consideradas acima são induzidas por seções locais $\sigma$ de $P$, conforme a equação (2.36), chegamos à conclusão de que uma seção

$$
\sigma: U \longrightarrow P
$$

de $P$ sobre $U$ em conjunto com uma carta

$$
x: U \longrightarrow U_{0}
$$

de $U$, onde $U_{0}$ é uma vizinhança aberta de $0 \mathrm{em} \mathbb{R}^{n}$, induz uma trivialização

$$
\begin{aligned}
& J^{k} \Phi_{\sigma}^{x}:\left.\quad J^{k}\left(P \times_{G} Q\right)\right|_{U} \quad \longrightarrow \quad U \times J_{0}^{k}\left(\mathbb{R}^{n}, Q\right) \\
& j_{m}^{k} \varphi \quad \longmapsto\left(m, j_{0}^{k}\left(\varphi_{\sigma} \circ x^{-1} \circ \tau_{x^{-1}(m)}\right)\right)
\end{aligned}
$$

de $J\left(P \times_{G} Q\right)$ sobre $U$, onde a aplicação $\varphi_{\sigma} \in C^{\infty}(U, Q)$ representa a seção $\varphi \in \Gamma\left(P \times\left._{G} Q\right|_{U}\right)$ conforme

$$
\varphi(m)=\left[\sigma(m), \varphi_{\sigma}(m)\right] \quad \text { para } m \in U .
$$

Exemplo 2.6 Considere a situação em que a fibra típica $Q$ é o próprio grupo estrutural $G$, como no Exemplo 2.1. Usando a ação de $G$ em si mesmo por translações à esquerda, definida pela equação (2.41), a trivialização induzida (2.54) assume a forma

$$
\begin{aligned}
& J^{k} \Phi_{\sigma}^{x}:\left.\quad J^{k} P\right|_{U} \quad \longrightarrow \quad U \times J_{0}^{k}\left(\mathbb{R}^{n}, G\right) \\
& j_{m}^{k}(\sigma \cdot g) \longmapsto\left(m, j_{0}^{k}\left(g \circ x^{-1} \circ \tau_{x^{-1}(m)}\right)\right)
\end{aligned}
$$

onde $g \in C^{\infty}(U, G)$, enquanto que usando a ação de $G$ em si mesmo por conjugação, definida pela equação (2.42), a trivialização induzida (2.54) assume a forma

$$
\begin{aligned}
& J^{k} \Phi_{\sigma}^{x}:\left.\quad J^{k}\left(P \times{ }_{G} G\right)\right|_{U} \longrightarrow \quad U \times J_{0}^{k}\left(\mathbb{R}^{n}, G\right) \\
& j_{m}^{k} g \quad \longmapsto\left(m, j_{0}^{k}\left(g_{\sigma} \circ x^{-1} \circ \tau_{x^{-1}(m)}\right)\right)
\end{aligned}
$$

onde a aplicação $g_{\sigma} \in C^{\infty}(U, G)$ representa a seção $g \in \Gamma\left(P \times\left._{G} G\right|_{U}\right)$ conforme

$$
g(m)=\left[\sigma(m), g_{\sigma}(m)\right] \quad \text { para } m \in U .
$$




\subsubsection{Grupos de jatos}

Retornando ao caso especial em que $M=\mathbb{R}^{n}$ e o ponto $m$ de $M$ é a origem de $\mathbb{R}^{n}$, suporemos ainda que a variedade alvo seja um grupo de Lie $G$ - uma situação que será de grande importância para o presente trabalho. Neste caso, podemos utilizar translações à direita ${ }^{2}$ para mostrar que $J_{0}^{k}\left(\mathbb{R}^{n}, G\right)$ é um fibrado (vetorial) trivial: de fato, usando o logaritmo ln (o inverso da exponencial exp : $\mathfrak{g} \rightarrow G$ ) como sistema de coordenadas para $G$ em torno de 1 , obtemos uma trivialização explícita

$$
\begin{array}{rlr}
J_{0}^{k}\left(\mathbb{R}^{n}, G\right) & \longrightarrow & G \times J_{0}^{k}\left(\mathbb{R}^{n}, \mathfrak{g}\right)_{0}=G \times \bigoplus_{j=1}^{k} L_{s}^{j}\left(\mathbb{R}^{n}, \mathfrak{g}\right) \\
j_{0}^{k} g & \longmapsto & j_{0}^{k}\left(\ln \left(g g(0)^{-1}\right)\right)=\left(g(0), D_{0} \ln \left(g g(0)^{-1}\right), \ldots, D_{0}^{k} \ln \left(g g(0)^{-1}\right)\right)
\end{array}
$$

que generaliza a trivialização bem conhecida

$$
\begin{aligned}
& T G \longrightarrow G \times \mathfrak{g} \\
& (g, \dot{g}) \stackrel{\cong}{\longmapsto}\left(g, \dot{g} g^{-1}\right)
\end{aligned}
$$

do fibrado tangente $T G$ de $G$. Por outro lado, $J_{0}^{k}\left(\mathbb{R}^{n}, G\right)$ também é um grupo de Lie em relação à multiplicação

$$
\begin{aligned}
& J_{0}^{k}\left(\mathbb{R}^{n}, G\right) \times J_{0}^{k}\left(\mathbb{R}^{n}, G\right) \longrightarrow J_{0}^{k}\left(\mathbb{R}^{n}, G\right) \\
& \left(j_{0}^{k} g_{1}, j_{0}^{k} g_{2}\right) \quad \longmapsto j_{0}^{k} g_{1} j_{0}^{k} g_{2}
\end{aligned}
$$

definida por

$$
j_{0}^{k} g_{1} j_{0}^{k} g_{2}=j_{0}^{k}\left(g_{1} g_{2}\right),
$$

para a qual o jato da função constante $=1$ configura uma unidade, e à inversão

$$
\begin{aligned}
J_{0}^{k}\left(\mathbb{R}^{n}, G\right) & \longrightarrow J_{0}^{k}\left(\mathbb{R}^{n}, G\right) \\
j_{0}^{k} g & \longmapsto\left(j_{0}^{k} g\right)^{-1}
\end{aligned}
$$

definida por

$$
\left(j_{0}^{k} g\right)^{-1}=j_{0}^{k}\left(g^{-1}\right),
$$

sendo que estas prescrições estão bem definidas devido à regra da cadeia, segundo a qual o $k$-jato de $g_{1} g_{2}$ e de $g^{-1}$ em 0 depende, respectivamente, apenas dos $k$-jatos de $g_{1}$ e $g_{2}$ e do $k$-jato de $g$ em 0 . Do mesmo modo, uma ação do grupo de Lie $G$ em uma variedade $Q$ induz uma ação

$$
\begin{array}{cl}
J_{0}^{k}\left(\mathbb{R}^{n}, G\right) \times J_{0}^{k}\left(\mathbb{R}^{n}, Q\right) & \longrightarrow J_{0}^{k}\left(\mathbb{R}^{n}, Q\right) \\
\left(j_{0}^{k} g, j_{0}^{k} q\right) & \longmapsto j_{0}^{k} g \cdot j_{0}^{k} q
\end{array}
$$

\footnotetext{
${ }^{2}$ Assim como poderíamos também usar translações à esquerda: é meramente uma questão de convenção.
} 
definida por

$$
j_{0}^{k} g \cdot j_{0}^{k} q=j_{0}^{k}(g \cdot q),
$$

sendo que esta prescrição está bem definida devido à regra da cadeia, segundo a qual o $k$-jato de $g \cdot q$ em 0 depende apenas dos $k$-jatos de $g$ e $q$ em 0 . (Para uma formulação explícita da regra da cadeia para derivadas de ordem superior, em termos de representações locais, veja $[2$, Teorema 1.4, p. 3].) Novamente, estas propriedades generalizam as propriedades correspondentes de $T G$ e $T Q$ explicadas na Seção 1.1.2, às quais se reduzem no caso especial em que $n=1$ e $k=1$.

Para uso posterior, queremos registrar a forma explícita do produto (2.61-2.62) em termos da trivialização (2.59) nos casos de interesse, $k=1$ e $k=2$. Para tanto, observamos primeiro que nestes casos, a inversa da trivialização (2.59) é dada por

$$
\begin{aligned}
& G \times L\left(\mathbb{R}^{n}, \mathfrak{g}\right) \quad J_{0}^{1}\left(\mathbb{R}^{n}, G\right) \\
& (g, \alpha) \quad \longmapsto j_{0}^{1}(u \mapsto \exp (\alpha(u)) g)
\end{aligned}
$$

e por

$$
\begin{array}{clc}
G \times L\left(\mathbb{R}^{n}, \mathfrak{g}\right) \times L_{s}^{2}\left(\mathbb{R}^{n}, \mathfrak{g}\right) & \longmapsto & J_{0}^{2}\left(\mathbb{R}^{n}, G\right) \\
(g, \alpha, \beta) & \cong j_{0}^{2}\left(u \mapsto \exp \left(\alpha(u)+\frac{1}{2} \beta(u, u)\right) g\right)
\end{array}
$$

respectivamente, e fazemos uso da fórmula

$$
\exp (s X) \exp (t Y)=\exp \left(s X+t Y+\frac{1}{2} s t[X, Y]+O\left((s+t)^{3}\right)\right) .
$$

para concluir que o produto em $G \times L\left(\mathbb{R}^{n}, \mathfrak{g}\right)$ que corresponde ao produto em $J_{0}^{1}\left(\mathbb{R}^{n}, G\right)$ é dado por

$$
\begin{gathered}
\left(g_{1}, \alpha_{1}\right)\left(g_{2}, \alpha_{2}\right)=\left(g_{1} g_{2}, \alpha_{1}+\operatorname{Ad}\left(g_{1}\right) \circ \alpha_{2}\right) \\
\operatorname{para}\left(g_{1}, \alpha_{1}\right),\left(g_{2}, \alpha_{2}\right) \in G \times L\left(\mathbb{R}^{n}, \mathfrak{g}\right) .
\end{gathered}
$$

Isso decorre do cálculo da primeira derivada na origem 0 de $\mathbb{R}^{n}$ da seguinte função de $u \in \mathbb{R}^{n}$ :

$$
\ln \left(\exp \left(\alpha_{1}(u)\right) g_{1} \exp \left(\alpha_{2}(u)\right) g_{1}^{-1}\right)=\alpha_{1}(u)+\operatorname{Ad}\left(g_{1}\right) \circ \alpha_{2}(u)+O\left(|u|^{2}\right) .
$$

Note que sob a correspondência

$$
\alpha_{\mu} \longleftrightarrow \partial_{\mu} g g^{-1}
$$

a fórmula (2.70) é facilmente deduzida se aplicarmos a regra do produto para calcular a expressão

$$
\partial_{\mu}\left(g_{1} g_{2}\right)\left(g_{1} g_{2}\right)^{-1}=\partial_{\mu} g_{1} g_{1}^{-1}+g_{1}\left(\partial_{\mu} g_{2} g_{2}^{-1}\right) g_{1}^{-1} .
$$

Desse modo vemos que $G \ltimes L\left(\mathbb{R}^{n}, \mathfrak{g}\right)$ é o produto semidireto $G \ltimes L\left(\mathbb{R}^{n}, \mathfrak{g}\right)$ de $G$ e $L\left(\mathbb{R}^{n}, \mathfrak{g}\right)$ (onde $L\left(\mathbb{R}^{n}, \mathfrak{g}\right)$ é considerado como grupo abeliano com respeito à adição) em relação à ação de $G$ por automorfismos de $L\left(\mathbb{R}^{n}, \mathfrak{g}\right)$ definida pela representação adjunta em $\mathfrak{g}$ combinada 
com a representação trivial em $\mathbb{R}^{n}$. Assim como ocorre com $T G$ (caso visto na Seção 1.1.2, ao qual o presente se reduz quando $n=1), G \ltimes L\left(\mathbb{R}^{n}, \mathfrak{g}\right)$ contém dois subgrupos: uma parte global, que é o grupo original $G$, e uma parte local, que é o subgrupo abeliano normal $L\left(\mathbb{R}^{n}, \mathfrak{g}\right)$. Obviamente, a correspondente álgebra de Lie é então a soma semidireta $\mathfrak{g} \ltimes L\left(\mathbb{R}^{n}, \mathfrak{g}\right)$, com comutador dado por

$$
\begin{gathered}
{\left[\left(X_{1}, \alpha_{1}\right),\left(X_{2}, \alpha_{2}\right)\right]=\left(\left[X_{1}, X_{2}\right], u \mapsto\left[X_{1}, \alpha_{2}(u)\right]-\left[X_{2}, \alpha_{1}(u)\right]\right)} \\
\operatorname{para}\left(X_{1}, \alpha_{1}\right),\left(X_{2}, \alpha_{2}\right) \in \mathfrak{g} \ltimes L\left(\mathbb{R}^{n}, \mathfrak{g}\right), u \in \mathbb{R}^{n} .
\end{gathered}
$$

De modo semelhante, fazemos uso da fórmula (2.69) para concluir que o produto em $G \times L\left(\mathbb{R}^{n}, \mathfrak{g}\right) \times L_{s}^{2}\left(\mathbb{R}^{n}, \mathfrak{g}\right)$ que corresponde ao produto em $J_{0}^{2}\left(\mathbb{R}^{n}, G\right)$ é dado por

$$
\begin{gathered}
\left(g_{1}, \alpha_{1}, \beta_{1}\right)\left(g_{2}, \alpha_{2}, \beta_{2}\right)=\left(g_{1} g_{2}, \alpha_{1}+\operatorname{Ad}\left(g_{1}\right) \circ \alpha_{2}, \beta_{1}+\left[\alpha_{1}, \operatorname{Ad}\left(g_{1}\right) \circ \alpha_{2}\right]_{s}+\operatorname{Ad}\left(g_{1}\right) \circ \beta_{2}\right)_{(2.73} \\
\text { para }\left(g_{1}, \alpha_{1}, \beta_{1}\right),\left(g_{2}, \alpha_{2}, \beta_{2}\right) \in G \times L\left(\mathbb{R}^{n}, \mathfrak{g}\right) \times L_{s}^{2}\left(\mathbb{R}^{n}, \mathfrak{g}\right),
\end{gathered}
$$

onde

$$
\begin{gathered}
{\left[\alpha_{1}, \alpha_{2}\right]_{s}\left(u_{1}, u_{2}\right)=\frac{1}{2}\left(\left[\alpha_{1}\left(u_{1}\right), \alpha_{2}\left(u_{2}\right)\right]+\left[\alpha_{1}\left(u_{2}\right), \alpha_{2}\left(u_{1}\right)\right]\right)} \\
\text { para } \alpha_{1}, \alpha_{2} \in L\left(\mathbb{R}^{n}, \mathfrak{g}\right), u_{1}, u_{2} \in \mathbb{R}^{n} .
\end{gathered}
$$

Isso decorre do cálculo das primeiras duas derivadas na origem 0 de $\mathbb{R}^{n}$ da seguinte função de $u \in \mathbb{R}^{n}$ :

$$
\begin{gathered}
\ln \left(\exp \left(\alpha_{1}(u)+\frac{1}{2} \beta_{1}(u, u)\right) g_{1} \exp \left(\alpha_{2}(u)+\frac{1}{2} \beta_{2}(u, u)\right) g_{1}^{-1}\right) \\
=\alpha_{1}(u)+\frac{1}{2} \beta_{1}(u, u)+\alpha_{2}(u)+\frac{1}{2} \beta_{2}(u, u) \\
\quad+\frac{1}{2}\left[\operatorname{Ad}\left(g_{2}^{-1}\right)\left(\alpha_{1}(u)\right), \alpha_{2}(u)\right]+O\left(|u|^{3}\right) .
\end{gathered}
$$

Note que sob a correspondência

$$
\alpha_{\mu} \longleftrightarrow \partial_{\mu} g g^{-1} \quad, \quad \beta_{\mu \nu} \longleftrightarrow \frac{1}{2}\left(\partial_{\mu}\left(\partial_{\nu} g g^{-1}\right)+\partial_{\nu}\left(\partial_{\mu} g g^{-1}\right)\right)
$$

a fórmula (2.73) é facilmente deduzida se aplicarmos a regra do produto para calcular a expressão

$$
\begin{aligned}
& \partial_{\mu}\left(\partial_{\nu}\left(g_{1} g_{2}\right)\left(g_{1} g_{2}\right)^{-1}\right)=\partial_{\mu}\left(\partial_{\nu} g_{1} g_{1}^{-1}+g_{1}\left(\partial_{\nu} g_{2} g_{2}^{-1}\right) g_{1}^{-1}\right) \\
& \quad=\partial_{\mu}\left(\partial_{\nu} g_{1} g_{1}^{-1}\right)+\left[\partial_{\mu} g_{1} g_{1}^{-1}, g_{1}\left(\partial_{\nu} g_{2} g_{2}^{-1}\right) g_{1}^{-1}\right]+g_{1} \partial_{\mu}\left(\partial_{\nu} g_{2} g_{2}^{-1}\right) g_{1}^{-1} .
\end{aligned}
$$

De forma análoga, registramos para uso posterior a forma explícita da ação (2.65-2.66), para o caso $k=1$, em termos das identificações (2.48) e (2.67):

$$
\begin{gathered}
(g, \alpha) \cdot(q, v)=\left(g \cdot q, u \mapsto g \cdot v(u)+\alpha(u)_{Q}(g \cdot q)\right) \\
\operatorname{para}(g, \alpha) \in G \times L\left(\mathbb{R}^{n}, \mathfrak{g}\right), q \in Q, v \in L\left(\mathbb{R}^{n}, T_{q} Q\right), u \in \mathbb{R}^{n} .
\end{gathered}
$$


Mais uma vez, sob a correspondência

$$
\alpha_{\mu} \longleftrightarrow \partial_{\mu} g g^{-1}, \quad v_{\mu} \longleftrightarrow \partial_{\mu} q
$$

esta fórmula é facilmente deduzida se aplicarmos a regra do produto para calcular a expressão

$$
\partial_{\mu}(g \cdot q)=g \cdot \partial_{\mu} q+\left(\partial_{\mu} g g^{-1}\right) \cdot(g \cdot q) .
$$

\subsubsection{Fibrado dos jatos de primeira ordem}

Uma situação especial que merece destaque é o caso $k=1$, no qual sempre omitiremos o índice 1, exceto quando houver perigo de confusão. Este caso é particularmente importante porque a grande maioria dos modelos, na teoria dos campos assim como na mecânica, se encaixa num formalismo de primeira ordem, cujas equações de movimento são equações diferenciais de segunda ordem. Numa abordagem geométrica, o fibrado dos jatos de primeira ordem $J E$ do fibrado de configuração $E$ (isto é, do fibrado $E$ sobre o espaço-tempo $M$ cujas seções representam os campos da teoria em questão), na teoria dos campos, tem um papel análogo ao do fibrado tangente $T Q$ do espaço de configuração $Q$, na mecânica.

\section{$J E$ como subfibrado afim de $L\left(\pi^{*} T M, T E\right)$}

Tendo em vista que, para $k=1$, a relação de equivalência $\sim_{k, m}$ definida na eq. (2.43) é dada por

$$
\varphi_{1} \sim_{1, m} \varphi_{2} \Longleftrightarrow \varphi_{1}(m)=\varphi_{2}(m) \quad \text { e } T_{m} \varphi_{1}=T_{m} \varphi_{2},
$$

e observando que a aplicação tangente $T \varphi: T M \rightarrow T E$ a uma seção $\varphi: M \rightarrow E$ de $E$ deve satisfazer $T \pi \circ T \varphi=\mathrm{id}_{T M}$, já que $\pi \circ \varphi=\mathrm{id}_{M}$, podemos identificar $J E$ com um subfibrado afim do fibrado vetorial $L\left(\pi^{*} T M, T E\right)$ sobre $E$, onde $\pi^{*} T M$ denota o pull-back do fibrado tangente $T M$ à base $M$ pela projeção $\pi$, da seguinte maneira: dado um ponto $m$ de $M$ e um ponto $e$ na fibra $E_{m}$ de $E$ sobre $m$, a fibra $J_{e} E$ de $J E$ em relação à projeção alvo é

$$
J_{e} E=\left\{u_{e} \in L\left(T_{m} M, T_{e} E\right) / T_{e} \pi \circ u_{e}=\operatorname{id}_{T_{m} M}\right\},
$$

enquanto que a fibra $J_{m} E$ de $J E$ em relação à projeção fonte é a união disjunta

$$
J_{m} E=\bigcup_{e \in E_{m}} J_{e} E
$$

de todas as fibras $J_{e} E$ de $J E$ em relação à projeção alvo. Obviamente, $J_{e} E$ é um subespaço afim do espaço vetorial $L\left(T_{m} M, T_{e} E\right) \cong T_{m}^{*} M \otimes T_{e} E$ e o espaço vetorial de diferenças correspondente é o espaço vetorial

$$
\overrightarrow{J_{e}} E=L\left(T_{m} M, V_{e} E\right) \cong T_{m}^{*} M \otimes V_{e} E .
$$


Assim como o fibrado dos jatos $J E$, o fibrado dos jatos linearizado $\vec{J} E$ assim definido também admite duas projeções diferentes, uma projeção alvo $\tau$ sobre $E$ e uma projeção fonte $\sigma$ sobre $M$ que é simplesmente a sua composição com a projeção original $\pi$, i.e., $\sigma=\pi \circ \tau$. É fácil mostrar que $J E$ e $\overrightarrow{J E}$ são fibrados sobre $M$ em relação à projeção fonte, em geral sem qualquer estrutura adicional, mas que $J E$ é um fibrado afim e $\vec{J} E$ é um fibrado vetorial sobre $E$ em relação à projeção alvo, sendo que o segundo é o o fibrado de diferenças do primeiro, canonicamente isomorfo ao fibrado vetorial das aplicações lineares do pull-back do fibrado tangente à base pela projeção $\pi$ para o fibrado vertical de $E$ :

$$
\vec{J} E=L\left(\pi^{*} T M, V E\right) \cong \pi^{*}\left(T^{*} M\right) \otimes V E .
$$

\section{Estrutura local}

A estrutura de $J E$ e de $\overrightarrow{J E}$ como fibrados sobre $M$ com respeito à projeção fonte (em geral sem nenhuma estrutura adicional), assim como a de $J E$ como fibrado afim e de $\vec{J} E$ como fibrado vetorial sobre $E$ com respeito à projeção alvo, podem ser vistas em termos de trivializações e coordenadas locais. Dadas coordenadas locais $x^{\mu}$ para $M$, coordenadas locais $q^{i}$ para a fibra típica $Q$ de $E$ e uma trivialização local de $E$, obtemos coordenadas locais induzidas $\left(x^{\mu}, q^{i}\right)$ para $E$ assim como coordenadas locais induzidas $\left(x^{\mu}, q^{i}, q_{\mu}^{i}\right)$ para $J E \subset L\left(\pi^{*}(T M), T E\right)$ e $\left(x^{\mu}, q^{i}, \vec{q}_{\mu}^{i}\right)$ para $\overrightarrow{J E} \subset L\left(\pi^{*}(T M), T E\right)$. Além disso, transformações de coordenadas locais $x^{\mu} \rightarrow x^{\prime \nu}$ para $M$ e $q^{i} \rightarrow q^{\prime j}$ para $Q$, juntamente com uma mudança de trivialização local de $E$, correspondem a uma transformação de coordenadas locais $\left(x^{\mu}, q^{i}\right) \rightarrow\left(x^{\prime \nu}, q^{\prime j}\right)$ para $E$, onde

$$
x^{\prime \nu}=x^{\prime \nu}\left(x^{\mu}\right) \quad, \quad q^{\prime j}=q^{\prime j}\left(x^{\mu}, q^{i}\right) .
$$

É fácil ver que as transformações de coordenadas locais induzidas $\left(x^{\mu}, q^{i}, q_{\mu}^{i}\right) \rightarrow\left(x^{\prime \nu}, q^{\prime j}, q_{\nu}^{\prime j}\right)$ para $J E$ e $\left(x^{\mu}, q^{i}, \vec{q}_{\mu}^{i}\right) \rightarrow\left(x^{\prime \nu}, q^{\prime j}, \vec{q}_{\nu}^{\prime j}\right)$ para $\overrightarrow{J E}$ são dadas por

$$
q_{\nu}^{\prime j}=\frac{\partial x^{\mu}}{\partial x^{\prime \nu}} \frac{\partial q^{\prime j}}{\partial q^{i}} q_{\mu}^{i}+\frac{\partial x^{\mu}}{\partial x^{\prime \nu}} \frac{\partial q^{\prime j}}{\partial x^{\mu}},
$$

$\mathrm{e}$

$$
\vec{q}_{\nu}^{\prime j}=\frac{\partial x^{\mu}}{\partial x^{\prime \nu}} \frac{\partial q^{\prime j}}{\partial q^{i}} \vec{q}_{\mu}^{i}
$$

Isto torna claro que $J E$ é um fibrado afim sobre $E$ com fibrado vetorial de diferenças $\vec{J} E$ dado pela eq. (2.82).

O fato de que o fibrado dos jatos (de primeira ordem) tem a estrutura adequada para incorporar derivadas (de primeira ordem) se torna mais claro notando-se que uma seção global $\varphi$ de $E$ sobre $M$ naturalmente induz uma seção global $j \varphi$ de $J E$ sobre $M$ dada por

$$
j \varphi(m)=T_{m} \varphi \in J_{\varphi(m)} E \quad \text { para } m \in M .
$$


Na literatura matemática, $j \varphi$ é chamado de prolongamento (de primeira ordem) de $\varphi$, mas seria mais intuitivo simplesmente chamá-lo de derivada de $\varphi$, já que nas coordenadas locais usadas acima,

$$
j \varphi(m)=\left(x^{\mu}(m), \varphi^{i}(m), \partial_{\mu} \varphi^{i}(m)\right),
$$

De modo similar, pode-se mostrar que o fibrado dos jatos linearizado de um fibrado tem a estrutura adequada para incorporar derivadas covariantes de seções, com respeito a uma conexão qualquer.

\section{Homomorfismos induzidos}

Dados fibrados gerais $E$ sobre $M$ com projeção $\pi$ e $E^{\prime}$ sobre $M^{\prime}$ com projeção $\pi^{\prime}$, um homomorfismo de fibrados $(f, \check{f})$ de $E$ em $E^{\prime}$ tal que a aplicação $\check{f}$ de $M$ em $M^{\prime}$ é um difeomorfismo induz um homomorfismo de fibrados afins $(J f, f)$ de $J E$ em $J E^{\prime}$ e um homomorfismo de fibrados vetoriais $(\vec{J} f, f)$ de $\overrightarrow{J E}$ em $\vec{J} E^{\prime}$ do seguinte modo: Dados pontos e em $E$ com ponto base $m=\pi(e)$ em $M$ e $e^{\prime}$ em $E^{\prime}$ com ponto base $m^{\prime}=\pi^{\prime}\left(e^{\prime}\right)$ em $M^{\prime}$ tais que $f(e)=e^{\prime}$, considerando as fibras $J_{e} E$ de $J E$ e $\vec{J}_{e} E$ de $\vec{J} E$ sobre $e$ como subespaços de $L\left(T_{m} M, T_{e} E\right)$ e as fibras $J_{e^{\prime}} E^{\prime}$ de $J E^{\prime}$ e $\vec{J}_{e^{\prime}} E^{\prime}$ de $\vec{J} E^{\prime}$ sobre $e^{\prime}$ como subespaços de $L\left(T_{m^{\prime}} M^{\prime}, T_{e^{\prime}} E^{\prime}\right)$, temos

$$
J_{e} f\left(u_{e}\right)=T_{e} f \circ u_{e} \circ\left(T_{m} \check{f}\right)^{-1} \quad, \quad \vec{J}_{e} f\left(\vec{u}_{e}\right)=T_{e} f \circ \vec{u}_{e} \circ\left(T_{m} \check{f}\right)^{-1} .
$$

Ademais, usando a regra da cadeia, que expressa o fato de que $T$ é um funtor (o funtor tangente, que leva a categoria das variedades para a categoria dos fibrados vetoriais), concluímos que $J$ e $\vec{J}$ também são funtores, que levam a categoria dos fibrados gerais, com morfismos restritos a homomorfismos de fibrados que induzem difeomorfismos entre as respectivas variedades base, para a categoria dos fibrados afins e para a categoria dos fibrados vetoriais, respectivamente.

\section{Jatos de um produto em fibras}

A seguir, estabelecemos uma proposição, bastante usada no Capítulo 3, que pode ser considerada o análogo, para fibrados, do fato de que o fibrado tangente de um produto cartesiano de duas variedades é canonicamente isomorfo ao produto cartesiano dos fibrados tangentes dos dois fatores $(T(X \times Y) \cong T X \times T Y)$. Sejam $E$ e $F$ dois fibrados sobre a mesma variedade base $M$ e considere seu produto em fibras $E \times_{M} F$. Tendo em vista que existe uma bijeção natural entre seções locais de $E \times_{M} F$ e pares de seções locais de $E$ e de $F$, todas definidas na mesma vizinhança aberta (arbitrária) de um mesmo ponto (arbitrário) de $M$, obtemos 
Proposição 2.1 Dados fibrados E e F sobre uma mesma variedade base $M$, existe um isomorfismo estrito canônico

$$
J\left(E \times_{M} F\right) \cong J E \times_{M} J F
$$

como fibrados sobre $M$ e como fibrados afins sobre $E \times{ }_{M} F$.

\subsubsection{Fibrado dos jatos de segunda ordem (por iteração)}

O fibrado dos jatos de segunda ordem pode ser definido ou diretamente, como no início desta seção, ou por meio de um procedimento iterativo, método que passamos a descrever agora. Partindo do fibrado dos jatos de primeira ordem $J E$ de $E$, visto como um fibrado sobre $M$, consideramos o seu fibrado dos jatos de primeira ordem $J J E$ e definimos, em um primeiro passo, o fibrado dos jatos de segunda ordem semiholônomo $\bar{J}^{2} E$ de $E$ como sendo o subfibrado de $J J E$ dado por

$$
\bar{J}^{2} E=\left\{\gamma \in J J E \mid \tau_{J E}(\gamma)=J \tau_{E}(\gamma)\right\}
$$

onde $\tau_{J E}: J J E \rightarrow J E$ é a projeção alvo de $J J E$ e $J \tau_{E}: J J E \rightarrow J E$ é obtido aplicando o funtor $J$ à projeção alvo $\tau_{E}: J E \rightarrow E$ de $J E$, considerada como um homomorfismo estrito de fibrados sobre $M . \bar{J}^{2} E$ resulta ser um fibrado afim sobre $J E$, com fibrado vetorial de diferenças ${\overrightarrow{J^{2}}}^{2}=\tau_{J E}^{*}\left(\pi^{*}\left(T^{*} M \otimes T^{*} M\right) \otimes V E\right)$. Além disso, $\bar{J}^{2} E$ admite uma decomposição natural, como produto em fibras sobre $J E$, em uma parte simétrica e uma parte antissimétrica: a parte simétrica é o fibrado dos jatos de segunda ordem $J^{2} E$, que é um subfibrado afim de $\bar{J}^{2} E$ cujo fibrado vetorial de diferenças é $\tau_{J E}^{*}\left(\pi^{*}\left(\bigvee^{2} T^{*} M\right) \otimes V E\right)$, ao passo que a parte antissimétrica é o subfibrado vetorial $\tau_{J E}^{*}\left(\pi^{*}\left(\bigwedge^{2} T^{*} M\right) \otimes V E\right)$ de $\vec{J}^{2} E$ (veja também [27]):

Lema 2.1 Para um fibrado geral $E$ sobre $M$, o fibrado dos jatos de segunda ordem semiholônomo $\bar{J}^{2} E$ admite uma decomposição canônica

$$
\bar{J}^{2} E \cong J^{2} E \times_{J E} \tau_{J E}^{*}\left(\pi^{*}\left(\bigwedge^{2} T^{*} M\right) \otimes V E\right) .
$$

na categoria dos fibrados afins sobre JE.

Estas afirmações podem ser provadas, por exemplo, introduzindo-se coordenadas locais adaptadas $\left(x^{\mu}, q^{i}\right)$ para $E$ e considerando as coordenadas locais induzidas $\left(x^{\mu}, q^{i}, q_{\mu}^{i}\right)$ para $J E$ que, por sua vez, providenciam coordenadas locais induzidas $\left(x^{\mu}, q^{i}, q_{\mu}^{i}, r_{\mu}^{i}, q_{\mu \rho}^{i}\right)$ para JJE. Cálculos simples mostram então que os pontos de $\bar{J}^{2} E$ são caracterizados pela condição 
$q_{\mu}^{i}=r_{\mu}^{i}$ e os pontos de $J^{2} E$ pela condição adicional $q_{\mu \rho}^{i}=q_{\rho \mu}^{i}$. Além disso, uma transformação de coordenadas locais adaptadas $\left(x^{\mu}, q^{i}\right) \rightarrow\left(x^{\prime \nu}, q^{\prime j}\right)$ para $E$ induz uma transformação das coordenadas locais induzidas $\left(x^{\mu}, q^{i}, q_{\mu}^{i}, r_{\mu}^{i}, q_{\mu \rho}^{i}\right) \rightarrow\left(x^{\prime \nu}, q^{\prime j}, q_{\nu}^{\prime j}, r_{\nu}^{\prime j}, q_{\nu \sigma}^{\prime j}\right)$ para $J J E$, dada pela eq. (2.84) em conjunto com as equações

$$
r_{\nu}^{\prime j}=\frac{\partial x^{\mu}}{\partial x^{\prime \nu}} \frac{\partial q^{\prime j}}{\partial q^{i}} r_{\mu}^{i}+\frac{\partial x^{\mu}}{\partial x^{\prime \nu}} \frac{\partial q^{\prime j}}{\partial x^{\mu}},
$$

$\mathrm{e}$

$$
q_{\nu \sigma}^{\prime j}=\frac{\partial x^{\rho}}{\partial x^{\prime \sigma}} \frac{\partial q_{\nu}^{\prime j}}{\partial q_{\mu}^{i}} q_{\mu \rho}^{i}+\frac{\partial x^{\rho}}{\partial x^{\prime \sigma}} \frac{\partial q_{\nu}^{\prime j}}{\partial q^{i}} r_{\rho}^{i}+\frac{\partial x^{\rho}}{\partial x^{\prime \sigma}} \frac{\partial q_{\nu}^{\prime j}}{\partial x^{\rho}} .
$$

Derivando a eq. (2.84) com respeito a $q_{\mu}^{i}, q^{i}$ e $x^{\rho}$ e usando a relação

$$
\frac{\partial^{2} x^{\mu}}{\partial x^{\prime \sigma} \partial x^{\prime \nu}}=-\frac{\partial x^{\rho}}{\partial x^{\prime \sigma}} \frac{\partial x^{\kappa}}{\partial x^{\prime \nu}} \frac{\partial x^{\mu}}{\partial x^{\prime \lambda}} \frac{\partial^{2} x^{\prime \lambda}}{\partial x^{\rho} \partial x^{\kappa}},
$$

podemos reescrever a eq. (2.91) explicitamente na forma

$$
\begin{aligned}
q_{\nu \sigma}^{\prime j}= & \frac{\partial x^{\rho}}{\partial x^{\prime \sigma}} \frac{\partial x^{\mu}}{\partial x^{\prime \nu}} \frac{\partial q^{\prime j}}{\partial q^{i}} q_{\mu \rho}^{i} \\
& +\frac{\partial x^{\rho}}{\partial x^{\prime \sigma}} \frac{\partial x^{\mu}}{\partial x^{\prime \nu}} \frac{\partial^{2} q^{\prime j}}{\partial q^{i} \partial q^{k}} q_{\mu}^{k} r_{\rho}^{i}+\frac{\partial x^{\rho}}{\partial x^{\prime \sigma}} \frac{\partial x^{\mu}}{\partial x^{\prime \nu}} \frac{\partial^{2} q^{\prime j}}{\partial q^{i} \partial x^{\mu}} r_{\rho}^{i} \\
& -\frac{\partial x^{\rho}}{\partial x^{\prime \sigma}} \frac{\partial x^{\kappa}}{\partial x^{\prime \nu}} \frac{\partial x^{\mu}}{\partial x^{\prime \lambda}} \frac{\partial^{2} x^{\prime \lambda}}{\partial x^{\rho} \partial x^{\kappa}} \frac{\partial q^{\prime j}}{\partial q^{i}} q_{\mu}^{i}-\frac{\partial x^{\rho}}{\partial x^{\prime \sigma}} \frac{\partial x^{\kappa}}{\partial x^{\prime \nu}} \frac{\partial x^{\mu}}{\partial x^{\prime \lambda}} \frac{\partial^{2} x^{\prime \lambda}}{\partial x^{\rho} \partial x^{\kappa}} \frac{\partial q^{\prime j}}{\partial x^{\mu}} \\
& +\frac{\partial x^{\rho}}{\partial x^{\prime \sigma}} \frac{\partial x^{\mu}}{\partial x^{\prime \nu}} \frac{\partial^{2} q^{\prime j}}{\partial x^{\rho} \partial q^{i}} q_{\mu}^{i}+\frac{\partial x^{\rho}}{\partial x^{\prime \sigma}} \frac{\partial x^{\mu}}{\partial x^{\prime \nu}} \frac{\partial^{2} q^{\prime j}}{\partial x^{\rho} \partial x^{\mu}} .
\end{aligned}
$$

Em particular, as eqs (2.84) e (2.90) mostram que $q_{\mu}^{i}=r_{\mu}^{i}$ implica $q_{\nu}^{\prime j}=r_{\nu}^{\prime j}$ e de modo similar, a eq. (2.93) mostra que se $q_{\mu}^{i}=r_{\mu}^{i}$, então $q_{\mu \rho}^{i}=q_{\rho \mu}^{i}$ implica $q_{\nu \sigma}^{\prime j}=q_{\sigma \nu}^{\prime j}$, como requer a natureza global e independente de coordenadas da definição tanto de $\bar{J}^{2} E$ como um subfibrado de $J J E$ quanto de $J^{2} E$ como um subfibrado de $\bar{J}^{2} E$. Ademais, a eq. (2.93) também mostra que se $q_{\mu}^{i}=r_{\mu}^{i}$, então a lei de transformação $q_{\mu \rho}^{i} \rightarrow q_{\nu \sigma}^{j}$ se decompõe naturalmente nas leis de transformação separadas $q_{(\mu \rho)}^{i} \rightarrow q_{(\nu \sigma)}^{\prime j}$ para a parte simétrica e $q_{[\mu \rho]}^{i} \rightarrow q_{[\nu \sigma]}^{j j}$ para a parte antissimétrica: a primeira,

$$
\begin{aligned}
q_{(\nu \sigma)}^{\prime j}= & \frac{\partial x^{\rho}}{\partial x^{\prime \sigma}} \frac{\partial x^{\mu}}{\partial x^{\prime \nu}} \frac{\partial q^{\prime j}}{\partial q^{i}} q_{(\mu \rho)}^{i}+\frac{\partial x^{\rho}}{\partial x^{\prime \sigma}} \frac{\partial x^{\mu}}{\partial x^{\prime \nu}} \frac{\partial^{2} q^{\prime j}}{\partial q^{i} \partial q^{k}} q_{\mu}^{k} q_{\rho}^{i} \\
& +\frac{\partial x^{\rho}}{\partial x^{\prime \sigma}} \frac{\partial x^{\mu}}{\partial x^{\prime \nu}}\left(\frac{\partial^{2} q^{\prime j}}{\partial x^{\mu} \partial q^{i}} q_{\rho}^{i}+\frac{\partial^{2} q^{\prime j}}{\partial x^{\rho} \partial q^{i}} q_{\mu}^{i}-\frac{\partial x^{\kappa}}{\partial x^{\prime \lambda}} \frac{\partial^{2} x^{\prime \lambda}}{\partial x^{\rho} \partial x^{\mu}} \frac{\partial q^{\prime j}}{\partial q^{i}} q_{\kappa}^{i}\right) \\
& -\frac{\partial x^{\rho}}{\partial x^{\prime \sigma}} \frac{\partial x^{\kappa}}{\partial x^{\prime \nu}} \frac{\partial x^{\mu}}{\partial x^{\prime \lambda}} \frac{\partial^{2} x^{\prime \lambda}}{\partial x^{\rho} \partial x^{\kappa}} \frac{\partial q^{\prime j}}{\partial x^{\mu}}+\frac{\partial x^{\rho}}{\partial x^{\prime \sigma}} \frac{\partial x^{\mu}}{\partial x^{\prime \nu}} \frac{\partial^{2} q^{\prime j}}{\partial x^{\rho} \partial x^{\mu}},
\end{aligned}
$$


é a lei de transformação para $J^{2} E$ como um fibrado afim sobre $J E$, enquanto que a segunda,

$$
q_{[\nu \sigma]}^{\prime j}=\frac{\partial x^{\rho}}{\partial x^{\prime \sigma}} \frac{\partial x^{\mu}}{\partial x^{\prime \nu}} \frac{\partial q^{j}}{\partial q^{i}} q_{[\mu \rho]}^{i},
$$

é a lei de transformação para $\tau_{J E}^{*}\left(\pi^{*}\left(\bigwedge^{2} T^{*} M\right) \otimes V E\right)$ como um fibrado vetorial sobre $J E$. Para mais detalhes, ver [27, Capítulo 5].

A equivalência entre a definição tradicional do fibrado dos jatos de segunda ordem, descrita no início desta seção, e a presente é obtida observando que o jato iterado $j j \varphi$ de uma seção (local) $\varphi$ de $E$ assume valores não somente em $\bar{J}^{2} E$ mas inclusive em $J^{2} E$, em virtude da regra de Schwarz. Portanto, jatos de segunda ordem no sentido tradicional, isto é, classes de equivalência de seções (locais), onde a relação de equivalência é a igualdade entre as expansões de Taylor até segunda ordem, estão em correspondência biunívoca com jatos iterados de seções (locais). Ademais, uma seção global $\varphi$ de $E$ sobre $M$ naturalmente induz uma seção global $j^{2} \varphi$ de $J^{2} E$ sobre $M$ tal que nas coordenadas locais usadas acima

$$
j^{2} \varphi(m)=\left(x^{\mu}(m), \varphi^{i}(m), \partial_{\mu} \varphi^{i}(m), \partial_{\mu} \partial_{\nu} \varphi^{i}(m)\right),
$$

onde $\partial_{\mu}=\partial / \partial x^{\mu}$; isto é simbolicamente resumido pela notação $j^{2} \varphi=\left(\varphi, \partial \varphi, \partial^{2} \varphi\right)$.

Abstratamente, podemos representar jatos iterados e caracterizar os fibrados $\bar{J}^{2} E$ e $J^{2} E$ globalmente como subfibrados de $J J E$ através de um simples truque que permite escrever todas as seções do fibrado $J E$ sobre $M$, holônomas ou não, na forma $j \varphi$, onde no entanto $\varphi$ é uma seção não de $E$ e sim do pull-back $\operatorname{pr}_{1}^{*} E$ de $E$ pela primeira projeção $\operatorname{pr}_{1}: M \times M \rightarrow M$ : tais seções representam "famílias suaves de seções de $E$ parametrizadas pelos pontos de $M$ ". Explicitamente,

$$
(j \varphi)(m)=j_{m} \varphi(., m) \quad \text { para } m \in M .
$$

Assim, todo ponto de $J J E$ é da forma $j_{m}(j \varphi)$ onde $\varphi$ é uma seção local de $\operatorname{pr}_{1}^{*} E$ definida em uma vizinhança $U \times U$ do ponto $(m, m)$ de $M \times M$, e denotando por $\varphi_{\Delta}$ a correspondente seção local "diagonal" de $E$, definida na vizinhança $U$ do ponto $m$ de $M$ por $\varphi_{\Delta}\left(m^{\prime}\right)=\varphi\left(m^{\prime}, m^{\prime}\right)$, temos $\tau_{E} \circ j \varphi=\varphi_{\Delta}$ e portanto $j_{m}(j \varphi)$ pertence a $\bar{J}^{2} E$ se e somente se a seção local $\varphi$ pode ser escolhida de modo que

$$
j_{m} \varphi_{\Delta}=j_{m} \varphi(., m)
$$

e pertence a $J^{2} E$ se e somente se $\varphi$ pode ser escolhida como sendo independente do segundo argumento; neste caso, escrevemos $j_{m}^{2} \varphi$ em vez de $j_{m}(j \varphi)$. 


\subsection{Conexões}

Nesta seção, apresentamos as definições do conceito de conexão mais comumente encontradas na literatura (ver $[5,11,19-21,26]$ ) e mostramos como elas se relacionam. Nosso principal objetivo é estabelecer a ponte entre a definição encontrada na literatura de física teórica e a definição mais adequada ao formalismo utilizado aqui, onde campos são seções de algum fibrado sobre o espaço-tempo. Em particular, conexões principais, que são a representação matemática dos campos de calibre, devem ser vistas deste modo. Com essa ponte a nossa disposição, saberemos como traduzir os conceitos fundamentais desta área, como, por exemplo, curvatura ou transformações de calibre, para o presente formalismo.

Começamos pela definição da noção de uma conexão geral em um fibrado geral e depois passaremos a conexões principais em fibrados principais.

\subsubsection{Conexões gerais}

\section{Fibrado horizontal, projeção vertical, projeção horizontal}

Definição 2.7 Uma conexão geral em um fibrado geral $E$ sobre uma variedade base $M$ com projeção $\pi: E \rightarrow M$ é dada por qualquer um dos seguintes três conceitos (que são equivalentes entre si):

- um fibrado horizontal $H E$, que é um complemento direto do fibrado vertical $V E$ (o núcleo da tangente $T \pi: T E \rightarrow T M$ da projeção) em TE:

$$
T E=V E \oplus H E
$$

- uma projeção vertical $V: T E \rightarrow V E$, com núcleo $H E$;

- uma projeção horizontal $H: T E \rightarrow H E$, com núcleo VE.

Obviamente, $V+H=\mathrm{id}_{T E}$, sendo que $V$ é a projeção de TE sobre $V E$ ao longo de $H E e$ $H$ é a projeção de TE sobre $H E$ ao longo de $V E$.

\section{Levantamento horizontal}

Uma definição alternativa pode ser dada usando o conceito do fibrado dos jatos (de primeira ordem) $J E$ de $E$, que foi tratado na seção anterior, segundo afirma a seguinte proposição: 
Proposição 2.2 Uma conexão geral em um fibrado geral $E$ sobre uma variedade base $M$ com projeção $\pi: E \rightarrow M$ também é dada pelo conceito de levantamento horizontal, que pode ser interpretado como uma seção $\Gamma: E \rightarrow J E$ de $J E$ como fibrado afim sobre $E$ (isto é, em relação à projeção alvo).

De fato, uma tal seção $\Gamma: E \rightarrow J E$ associa a cada ponto $e$ de $E$ um jato $\Gamma(e) \in J_{e} E$, isto é, uma aplicação linear $\Gamma(e): T_{x} M \rightarrow T_{e} E$ cuja composição com a aplicação linear $T_{e} \pi: T_{e} E \rightarrow T_{x} M$ é a identidade em $T_{x} M:$

$$
T_{e} \pi \circ \Gamma(e)=\operatorname{id}_{T_{x} M} .
$$

Portanto, composição de $\Gamma(e) \operatorname{com} T_{e} \pi$ na outra ordem fornece uma projeção horizontal:

$$
H_{e}=\Gamma(e) \circ T_{e} \pi
$$

Reciprocamente, dado o fibrado horizontal $H E, \Gamma(e)$ será o inverso da restrição de $T_{e} \pi$ a $H_{e} E$. Note também que o levantamento horizontal em nível de campos vetoriais associa a cada campo vetorial $X$ sobre $M$ um campo vetorial $\Gamma(X)$ horizontal sobre $E$ definido por

$$
\Gamma(X)(e)=\Gamma(e)(X(\pi(e))) .
$$

Em coordenadas locais $\left(x^{\mu}, q^{i}, q_{\mu}^{i}\right)$ para $J E$ induzidas por coordenadas locais adaptadas $\left(x^{\mu}, q^{i}\right)$ para $E$, a seção $\Gamma$ é dada por funções $\Gamma_{\mu}^{i}$, i.e., por $q_{\mu}^{i}=\Gamma_{\mu}^{i}(x, q)$, de modo que os

$$
\Gamma\left(\partial_{\mu}\right)=\partial_{\mu}+\Gamma_{\mu}^{i} \partial_{i}
$$

formam uma base de campos vetoriais horizontais locais em $E$.

\section{Curvatura}

Definição 2.8 A curvatura de uma conexão geral em um fibrado geral E sobre uma variedade base $M$ com projeção $\pi: E \rightarrow M$ é a 2 -forma horizontal ${ }^{3} \Omega$ sobre $E$ a valores no fibrado vertical $V E$ de $E$ que associa a quaisquer dois campos vetoriais $X$ e $Y$ em $E$ a projeção vertical do colchete de Lie das suas projeções horizontais:

$$
\Omega(X, Y)=\left[X_{H}, Y_{H}\right]_{V} .
$$

Assim, a curvatura de uma conexão expressa a falta de integrabilidade do fibrado horizontal.

\footnotetext{
${ }^{3}$ Lembramos que formas diferenciais sobre o espaço total de um fibrado são chamadas horizontais se se anulam assim que inserimos um campo vertical.
} 
Um outra maneira de visualizar este conceito que se tornará útil mais tarde é a seguinte. Dada uma conexão em $E$ na forma de uma seção $\Gamma: E \rightarrow J E$ e considerando tanto $E$ como $J E$ como fibrados sobre $M$, podemos aplicar o funtor $J$ para definir a sua derivada $J \Gamma: J E \rightarrow J J E$, conforme a equação (2.86), e formar a composição $J \Gamma \circ \Gamma: E \rightarrow J J E$, que toma valores em $\bar{J}^{2} E$ pois

$$
\tau_{J E} \circ \Gamma \circ \Gamma=\Gamma \circ \tau_{E} \circ \Gamma=\Gamma \quad \text { e } \quad J \tau_{E} \circ \Gamma \circ \Gamma=J\left(\tau_{E} \circ \Gamma\right) \circ \Gamma=\Gamma .
$$

Então considerando $\Omega$ como seção do fibrado vetorial $\pi^{*}\left(\bigwedge^{2} T^{*} M\right) \otimes V E$ sobre $E$, temos que, a menos de um fator $-2, \Omega$ é a parte antissimétrica de $J \Gamma \circ \Gamma$.

Em coordenadas locais $\left(x^{\mu}, q^{i}, q_{\mu}^{i}\right)$ para $J E,\left(x^{\mu}, q^{i}, q_{\mu}^{i}, r_{\mu}^{i}, q_{\mu \rho}^{i}\right)$ para $J J E$ e $\left(x^{\mu}, q^{i}, q_{\mu}^{i}, q_{\mu \rho}^{i}\right)$ para $\bar{J}^{2} E$ induzidas por coordenadas locais adaptadas $\left(x^{\mu}, q^{i}\right)$ para $E$, temos

$$
\Omega_{\mu \nu}^{i} \partial_{i}=\Omega\left(\Gamma\left(\partial_{\mu}\right), \Gamma\left(\partial_{\nu}\right)\right)=\left[\partial_{\mu}+\Gamma_{\mu}^{j} \partial_{j}, \partial_{\nu}+\Gamma_{\nu}^{k} \partial_{k}\right],
$$

i.e.,

$$
\Omega_{\mu \nu}^{i}=\partial_{\mu} \Gamma_{\nu}^{i}-\partial_{\nu} \Gamma_{\mu}^{i}+\Gamma_{\mu}^{j} \partial_{j} \Gamma_{\nu}^{i}-\Gamma_{\nu}^{j} \partial_{j} \Gamma_{\mu}^{i} .
$$

Por outro lado, tendo em vista que a derivada $J \Gamma: J E \rightarrow J J E$ de $\Gamma: E \rightarrow J E$ é definida pela regra de que para $m \in M, e \in E$ e $u_{e} \in J_{e} E$, representado na forma $u_{e}=j_{m} \varphi$ onde $\varphi$ é uma seção local de $E$, definida em uma vizinhança de $m$ e satisfazendo $\varphi(m)=e$, $J_{e} \Gamma\left(u_{e}\right) \in J_{\Gamma(e)}(J E)$ será representado na forma $J_{e} \Gamma\left(u_{e}\right)=j_{m}(\Gamma \circ \varphi)$, então podemos aplicar a regra da cadeia a esta expressão para concluir que $J \Gamma$ levará o ponto com coordenadas $\left(x^{\mu}, q^{i}, q_{\mu}^{i}\right)$ no ponto com coordenadas $\left(x^{\mu}, q^{i}, \Gamma_{\mu}^{i}, q_{\mu}^{i}, \partial_{\rho} \Gamma_{\mu}^{i}+\Gamma_{\rho}^{j} \partial_{j} \Gamma_{\mu}^{i}\right)$ e portanto a seção composta $J \Gamma \circ \Gamma$ é dada pelas "derivadas horizontais" das funções $\Gamma_{\mu}^{i}$, i.e., por $q_{\mu \rho}^{i}=\left(\partial_{\rho} \Gamma_{\mu}^{i}+\Gamma_{\rho}^{j} \partial_{j} \Gamma_{\mu}^{i}\right)(x, q)$, com parte antissimétrica igual a $-2 \Omega_{\mu \rho}^{i}$.

\subsubsection{Conexões principais}

Definição 2.9 Uma conexão principal em um fibrado principal $P$ sobre uma variedade base $M$ com grupo estrutural $G$ é uma conexão geral em $P$ que é equivariante sob a ação de $G$ em $P$, ou mais exatamente, sob a ação induzida de $G$ em TP.

\section{Forma de conexão}

Outra definição do conceito de uma conexão principal, bastante útil por permitir aplicar o cálculo de formas diferenciais (a valores vetoriais) em variedades, pode ser dada usando o conceito de forma de conexão. Ela é baseada no fato de que o fibrado vertical $V P$ de um fibrado principal $P$ admite uma trivialização global canônica $G$-equivariante

$$
\begin{aligned}
P \times \mathfrak{g} & \geqq V P \\
(p, X) & \cong X^{P}(p)
\end{aligned},
$$


onde $\mathfrak{g}$ é a álgebra de Lie do grupo estrutural $G$ de $P$ e $X^{P}$ denota o campo fundamental sobre $P$ associado ao gerador $X$ em $\mathfrak{g}$ em relação à ação $(2.21)$ de $G$,

$$
X^{P}(p)=\left.\frac{d}{d t} p \cdot \exp t X\right|_{t=0} \quad \text { para } X \in \mathfrak{g}, p \in P .
$$

De fato, a aplicação (2.105) é obviamente um homomorfismo de fibrados vetoriais sobre $P$ que é $G$-equivariante no sentido de que

$$
X^{P}(p) \cdot g=\left(\operatorname{Ad}\left(g^{-1}\right) X\right)(p \cdot g) \quad \text { para } g \in G, X \in \mathfrak{g}, p \in P,
$$

onde fizemos uso da ação induzida de $G$ em $T P$ (e sua restrição a $V P$ ), e que define, em cada ponto $p$ de $P$, um isomorfismo linear entre $\mathfrak{g}$ e o espaço vertical $V_{p} P$, pois a ação (2.21) é livre e transitiva em cada fibra de $P$. Para uso posterior, notamos que tomando o produto tensorial com a identidade no fibrado cotangente de $P$, obtemos um isomorfismo global canônico $G$-equivariante

$$
\begin{array}{ccc}
L(T P, P \times \mathfrak{g}) & \geqq L(T P, V P) \\
A_{p} & \cong A_{p}^{P}(p)
\end{array}
$$

de fibrados vetoriais sobre $P$, e denotando por $\operatorname{Pr}(T P, V P)$ o subfibrado afim do fibrado vetorial $L(T P, V P)$ formado pelas projeções sobre $V P$ (i.e., pelos elementos de $L(T P, V P)$ cuja restrição a $V P$ é a identidade) e por $\operatorname{Pr}(T P, P \times \mathfrak{g})$ o subfibrado afim do fibrado vetorial $L(T P, P \times \mathfrak{g})$ que é a sua imagem inversa sob o isomorfismo (2.108), obtemos um isomorfismo global canônico $G$-equivariante

$$
\begin{array}{cl}
\operatorname{Pr}(T P, P \times \mathfrak{g}) & \longmapsto \operatorname{Pr}(T P, V P) \\
A_{p} & \cong A_{p}^{P}(p)
\end{array}
$$

de fibrados afins sobre $P$, onde empregamos a notação

$$
A_{p}^{P}(p)\left(u_{p}\right) \equiv\left(A_{p}\left(u_{p}\right)\right)^{P}(p) \quad \text { para } p \in P, A_{p} \in L\left(T_{p} P, \mathfrak{g}\right), u_{p} \in T_{p} P .
$$

Definição 2.10 Uma forma de conexão em um fibrado principal $P$ sobre uma variedade base $M$ com grupo estrutural $G$ é uma 1-forma $A$ em $P$ a valores na álgebra de Lie $\mathfrak{g}$ de $G$ que é equivariante sob a ação de $G$ e cuja restrição a VP coincide com a trivialização (2.105), isto é, uma seção $G$-equivariante do fibrado afim $\operatorname{Pr}(T P, P \times \mathfrak{g})$ introduzido acima.

A equivalência entre esta definição e as anteriores pode ser provada de várias maneiras, por exemplo, observando que sob o isomorfismo (2.109), $A$ corresponde à projeção vertical e portanto o fibrado horizontal é o núcleo de $A$. Outras correspondências serão estabelecidas logo abaixo. 


\section{Potenciais de calibre}

Em termos de um atlas de fibrado principal de $P$, dado por uma cobertura de $M$ por abertos $U_{\alpha}$ e por uma família de seções $\sigma_{\alpha}$ de $P$ sobre $U_{\alpha}$, com funções de transição $g_{\alpha \beta}: U_{\alpha} \cap U_{\beta} \rightarrow G$ definidas por $\sigma_{\beta}(m)=\sigma_{\alpha}(m) \cdot g_{\alpha \beta}(m)$ para $m \in U_{\alpha} \cap U_{\beta}$, uma forma de conexão $A$ sobre $P$ é representada por uma família de 1-formas $A^{(\alpha)}$ em $U_{\alpha}$ a valores em $\mathfrak{g}$, definidas por $A^{(\alpha)}=\sigma_{\alpha}^{*} A$, que satisfazem a seguinte lei de transformação:

$$
A^{(\beta)}=\operatorname{Ad}\left(g_{\alpha \beta}\right)^{-1} A^{(\alpha)}+g_{\alpha \beta}^{-1} d g_{\alpha \beta} .
$$

Reciprocamente, um conjunto de 1-formas $A^{(\alpha)}$ em $U_{\alpha}$ a valores em $\mathfrak{g}$ satisfazendo esta lei de transformação define uma única forma de conexão em $P$. É nesta forma local e dependente da escolha de calibre (expressa pela escolha das seções locais $\sigma_{\alpha}$ ) que conexões principais aparecem na física, nas teorias de calibre.

\subsubsection{Fibrado das conexões}

Finalmente, queremos apresentar mais uma definição do conceito de uma conexão principal, a saber como seção de um certo fibrado afim $C P$ sobre a variedade base $M$ chamado o fibrado das conexões $C P$ do fibrado principal $P$ : ele é definido como o quociente de um fibrado afim sobre $P$ munido de uma ação natural de $G$ induzida pela ação de $G$ em $P$.

Do ponto de vista do levantamento horizontal, este fibrado afim sobre $P$ é o fibrado dos jatos de primeira ordem de $P$, de modo que

$$
C P \cong J P / G,
$$

enquanto que do ponto de vista das formas de conexão, este fibrado afim sobre $P$ é o fibrado $\operatorname{Pr}(T P, P \times \mathfrak{g})$ introduzido acima, de modo que

$$
C P \cong \operatorname{Pr}(T P, P \times \mathfrak{g}) / G,
$$

o que implica, em ambos os casos, que seções equivariantes destes fibrados sobre $P$ correspondem a seções do fibrado $C P$ sobre $M$. Para efetuarmos cálculos concretos, no entanto, a realização de $C P$ como fibrado quociente é menos conveniente do que a seguinte construção direta das fibras de $C P$ como espaços afins de seções equivariantes ao longo das respectivas fibras de $P$ : para todo ponto $m$ da variedade base $M$, considere a restrição do fibrado afim $J P$ e do fibrado afim $\operatorname{Pr}(T P, P \times \mathfrak{g})$ à fibra $P_{m}$ de $P$ sobre $m$ e defina $(C P)_{m}$ como sendo o espaço afim das seções $G$-equivariantes deste fibrado, i.e.,

$$
(C P)_{m} \cong \Gamma_{E}\left(J P \mid P_{m}\right),
$$


cujos elementos denotaremos por $\Gamma_{m}^{A}$ ou

$$
(C P)_{m} \cong \Gamma_{E}\left(\operatorname{Pr}(T P, P \times \mathfrak{g}) \mid P_{m}\right)
$$

cujos elementos denotaremos por $A_{m}$; note que estes espaços são de dimensão finita porque $G$ age livre e transitivamente sobre cada fibra de $P$ e portanto uma seção equivariante ao longo de uma fibra é completamente determinada por seu valor em um único ponto desta fibra. A equivalência destas duas definições pode ser estabelecida lançando mão dos isomorfismos canônicos $G$-equivariantes

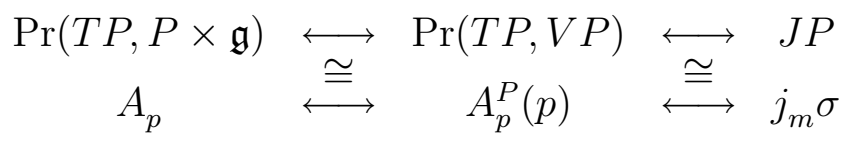

de fibrados afins sobre $P$ onde o primeiro é definido pelas equações (2.109) e (2.110) enquanto que o segundo é dado pela fórmula

$$
A_{p}^{P}(p)+j_{m} \sigma \circ T_{p} \rho=\operatorname{id}_{T_{p} P} \quad \operatorname{com} p=\sigma(m),
$$

que é equivalente à fórmula

$$
A_{p}^{P}(p) \circ j_{m} \sigma=0 \quad \operatorname{com} p=\sigma(m),
$$

pois esta condição significa que a imagem do jato $j_{m} \sigma$ é contida no núcleo do projetor $A_{p}^{P}(p)$, o que já é suficiente para garantir que $\operatorname{im} j_{m} \sigma=\operatorname{ker} A_{p}^{P}(p)$, por motivos dimensionais. Nestes termos, note a vantagem de usar a interpretação dos pontos de $C P$ dada pelas equações (2.113) e (2.114), em vez das equações (2.111) e (2.112): as seções equivariantes $\Gamma_{m}^{A}$ e $A_{m}$ providenciam, de uma vez só, toda uma família de jatos $\Gamma_{m}^{A}(p)$ e de projetores $A_{m}(p)$, onde $p$ percorre a fibra $P_{m}$, que constituem exatamente o conjunto de todos os representantes da sua respectiva classe de equivalência no espaço quociente pertinente, sendo que com esta notação, as fórmulas (2.116) e (2.117) que estabelecem a relação entre as duas assumem a forma

$$
A_{m}(p)^{P}(p)+\Gamma_{m}^{A}(p) \circ T_{p} \rho=\operatorname{id}_{T_{p} P} \quad \text { para } p \in P_{m},
$$

$\mathrm{e}$

$$
A_{m}(p)^{P}(p) \circ \Gamma_{m}^{A}(p)=0 \quad \text { para } p \in P_{m},
$$

respectivamente. De qualquer modo, evidencia-se que um elemento de $C P$ é uma conexão ao longo da respectiva fibra de $P$, e que vale a seguinte

Proposição 2.3 Uma conexão principal em um fibrado principal $P$ sobre uma variedade base $M$ com grupo estrutural $G$ também é dada por uma seção do fibrado das conexões CP de $P$, ou seja, um elemento do espaço afim

$$
\Gamma(C P) \cong \Gamma_{E}(J P) \cong \Gamma_{E}(\operatorname{Pr}(T P, P \times \mathfrak{g})) .
$$


É esta interpretação de conexões principais - como seções de um determinado fibrado sobre o espaço-tempo - que melhor expressa sua natureza como campos.

Para uso posterior, notamos também que devido aos isomorfismos (2.111) e (2.112), temos os seguintes diagramas comutativos:

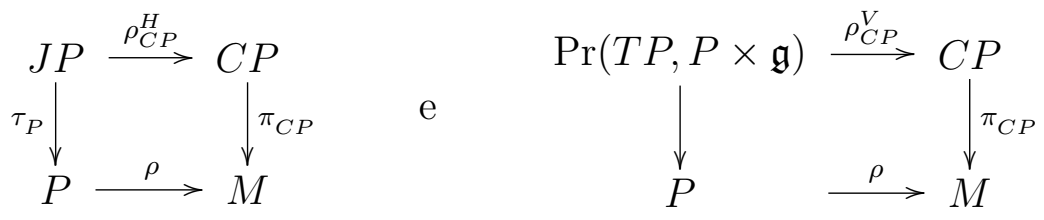

Observando que as ações de $G$ em $J P$ e em $\operatorname{Pr}(T P, P \times \mathfrak{g})$ são livres pois recobrem a ação de $G$ em $P$ que é livre, podemos concluir que $C P$ é uma variedade tal que (a) $C P$ é o espaço total de um fibrado afim sobre $M$ (já que $J P$ e $\operatorname{Pr}(T P, P \times \mathfrak{g}$ ) são espaços totais de fibrados afins sobre $P$ e $M=P / G)$ e (b) $J P$ e $\operatorname{Pr}(T P, P \times \mathfrak{g})$ são espaços totais de fibrados principais sobre $C P$ com grupo estrutural $G$, ou seja, as flechas verticais nos diagramas acima definem fibrados afins enquanto que as flechas horizontais definem fibrados principais com grupo estrutural $G$. Notamos também que isso implica que $J P$ e $\operatorname{Pr}(T P, P \times \mathfrak{g})$, vistos como fibrados sobre $M$, são canonicamente isomorfos ao produto em fibras de $P$ e $C P$ :

$$
J P \cong P \times{ }_{M} C P \quad \text { e } \quad \operatorname{Pr}(T P, P \times \mathfrak{g}) \cong P \times{ }_{M} C P .
$$

De fato, combinando as projeções canônicas de $J P$ e $\operatorname{Pr}(T P, P \times \mathfrak{g})$ sobre $P$ com as projeções quociente sobre $C P$, obtemos homomorfismos

$$
\begin{aligned}
& J P \quad \longrightarrow \quad P \times{ }_{M} C P \quad \text { e } \quad \operatorname{Pr}(T P, P \times \mathfrak{g}) \longrightarrow P \times_{M} C P \\
& j_{m} \sigma \longmapsto\left(\sigma(m),\left[j_{m} \sigma\right]\right) \quad \text { e } \quad A_{p} \quad \longmapsto \quad\left(p,\left[A_{p}\right]\right)
\end{aligned}
$$

de fibrados sobre $M$ que são isomorfismos pois $\left[j_{m} \tau\right]=\left[j_{m} \sigma\right]$ ou $\left[A_{p}\right]=\left[A_{q}\right]$ significa que existe $g \in G$ (que é único) tal que $j_{m} \tau=j_{m} \sigma \cdot g \equiv j_{m}(\sigma \cdot g)$ ou $A_{q}=A_{p} \cdot g$, o que implica em $\tau(m)=\sigma(m) \cdot g$ ou $q=p \cdot g$. Portanto,

$$
\begin{gathered}
\left(\tau(m),\left[j_{m} \tau\right]\right)=\left(\sigma(m),\left[j_{m} \sigma\right]\right) \Longrightarrow j_{m} \tau=j_{m} \sigma \\
\text { ou } \\
\left(q,\left[A_{q}\right]\right)=\left(p,\left[A_{p}\right]\right) \Longrightarrow A_{q}=A_{p}
\end{gathered}
$$

\section{Estrutura local}

Para construir trivializações locais explícitas do fibrado $C P$, definidas a partir de uma seção $\sigma$ do fibrado principal $P$ sobre um aberto $U$ da variedade base $M$, em conjunto com uma 
carta $x$ para $U$, como sempre, combinamos a trivialização (2.56) (para $k=1$ ) com a trivialização (2.59) (para $k=1$ ), cuja inversa é dada pela equação (2.67), para obter a seguinte trivialização de $J P$ sobre $U$,

$$
\begin{array}{cccc}
J \Phi_{\sigma}^{x}:\left.\quad J P\right|_{U} & \longrightarrow & U \times\left(G \times L\left(\mathbb{R}^{n}, \mathfrak{g}\right)\right) \\
j_{m}(\sigma \cdot g) & \longmapsto & \left(m, g(m),\left(\partial g g^{-1}\right)(m) \circ D_{x^{-1}(m)} x^{-1}\right)
\end{array},
$$

onde $g \in C^{\infty}(U, G)$ e $\partial g g^{-1} \in \Omega^{1}(U, \mathfrak{g})$ pode ser visto como o pull-back da forma de MaurerCartan invariante à direita em $G$ pela aplicação $g$; em coordenadas,

$$
\partial g g^{-1}=\left(\partial_{\mu} g g^{-1}\right) d x^{\mu} .
$$

Passando para o quociente pela ação de $G$, obtemos uma trivialização de $C P$ sobre $U$ que escreveremos na forma

$$
\begin{array}{cccc}
C \Phi_{\sigma}^{x}:\left.\quad C P\right|_{U} & \longrightarrow \quad U \times L\left(\mathbb{R}^{n}, \mathfrak{g}\right) \\
\Gamma_{m}^{A} \cong A_{m} & \longmapsto\left(m, A_{m}^{\sigma} \circ D_{x^{-1}(m)} x^{-1}\right)
\end{array}
$$

onde $A_{m}^{\sigma} \in L\left(T_{m} M, \mathfrak{g}\right)$ é explicitamente dada por qualquer uma das seguintes construções:

- Em termos de $A_{m} \in \Gamma_{E}\left(\operatorname{Pr}(T P, P \times \mathfrak{g}) \mid P_{m}\right), A_{m}^{\sigma}$ é simplesmente o pull-back de $A_{m}(\sigma(m)) \operatorname{com} \sigma$ :

$$
A_{m}^{\sigma}=\sigma^{*}\left(A_{m}(\sigma(m))\right) \equiv A_{m}(\sigma(m)) \circ T_{m} \sigma
$$

- Em termos de $\Gamma_{m}^{A} \in \Gamma_{E}\left(J P \mid P_{m}\right), A_{m}^{\sigma}$ é, a menos de um sinal, a derivada em $m$

$$
A_{m}^{\sigma}=-T_{m} g^{A, \sigma}
$$

de qualquer função $g^{A, \sigma} \in C^{\infty}(U, G)$ satisfazendo $g^{A, \sigma}(m)=1$ e tal que

$$
\Gamma_{m}^{A}(\sigma(m))=j_{m}\left(\sigma \cdot g^{A, \sigma}\right)
$$

Para provar que estas duas prescrições são de fato equivalentes, note que tendo em vista a condição $g^{A, \sigma}(m)=1$ em conjunto com a definição (2.106) dos campos fundamentais associados à ação de $G$ em $P$, temos para todo $u \in T_{m} M$,

$$
T_{m}\left(\sigma \cdot g^{A, \sigma}\right)(u)=T_{m} \sigma(u)+\left(T_{m} g^{A, \sigma}(u)\right)^{P}(\sigma(m)),
$$

ou se usamos a mesma notação como na equação (2.110),

$$
\begin{aligned}
\left(T_{m} g^{A, \sigma}\right)^{P}(\sigma(m)) & =T_{m}\left(\sigma \cdot g^{A, \sigma}\right)-T_{m} \sigma=\Gamma_{m}^{A}(\sigma(m))-T_{m} \sigma \\
& =\left(\Gamma_{m}^{A}(\sigma(m)) \circ T_{\sigma(m)} \rho-\mathrm{id}_{T_{\sigma(m)} P}\right) \circ T_{m} \sigma \\
& =-\left(A_{m}(\sigma(m))^{P}(\sigma(m)) \circ T_{m} \sigma,\right.
\end{aligned}
$$


mostrando que

$$
T_{m} g^{A, \sigma}=-A_{m}(\sigma(m)) \circ T_{m} \sigma
$$

Em nível de seções de $C P$ sobre $U$ (e revertendo o uso da carta $x$ ), esta trivialização induz um isomorfismo

$$
\begin{aligned}
\Gamma\left(\left.C P\right|_{U}\right) & \longmapsto \Omega^{1}(U, \mathfrak{g}) \\
\Gamma^{A} \cong A & \cong A^{\sigma}
\end{aligned}
$$

onde a relação entre $A^{\sigma}$ e $A$ é muitos simples,

$$
A^{\sigma}=\sigma^{*} A
$$

enquanto que a relação entre $A^{\sigma}$ e $\Gamma^{A}$ é mais complicada, devido ao fato de que, em geral, a seção $\Gamma^{A}$ não corresponde a uma seção holônoma de $J P$. No entanto, este problema pode ser evitado se admitirmos usar uma família de funções, parametrizada pelos próprios pontos de $U$, em vez de uma única função. Deste modo, representaremos $\Gamma^{A}$ por uma função $g^{A, \sigma} \in C^{\infty}(U \times U, G)$ satisfazendo $g^{A, \sigma}(m, m)=1$, conforme ${ }^{4}$

$$
\Gamma_{m}^{A}(\sigma(m))=j_{m}\left(\sigma \cdot g^{A, \sigma}(., m)\right)
$$

e então a relação entre $A^{\sigma}$ e $\Gamma^{A}$ é dada por

$$
A_{m}^{\sigma}=-T_{m} g^{A, \sigma}(., m)
$$

\subsubsection{Conexões associadas e derivada covariante}

Uma conexão principal em um fibrado principal $P$ sobre uma variedade base $M$ com grupo estrutural $G$ induz uma conexão em qualquer fibrado $P \times_{G} Q$ associado a $P$. Por exemplo, a projeção vertical $V_{P \times_{G} Q}: T\left(P \times_{G} Q\right) \rightarrow T\left(P \times_{G} Q\right)$ da conexão induzida pode ser construída a partir da projeção vertical $V_{P}: T P \rightarrow T P$ da conexão principal requerendo-se que o seguinte diagrama seja comutativo:

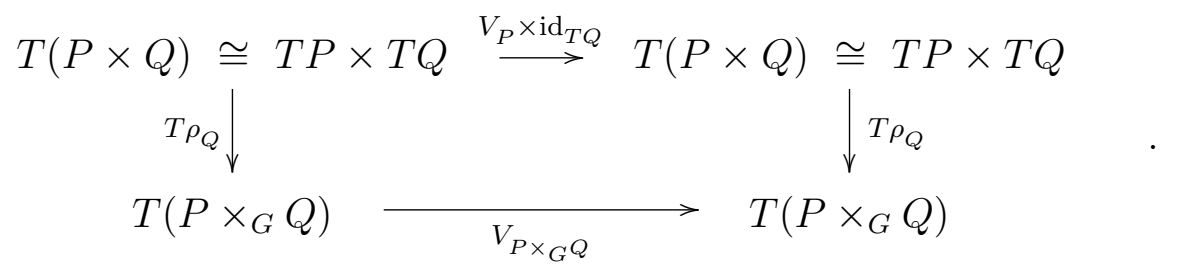

\footnotetext{
${ }^{4} \mathrm{Um}$ procedimento análogo para descrever jatos iterados foi discutido no final da Seção 2.2.5.
} 
Para provar que de fato esta prescrição define uma conexão no fibrado associado, note que pontualmente, para todo ponto $p \in P$ e todo ponto $q \in Q$, este diagrama assume a forma

$$
\begin{aligned}
& T_{p} P \oplus T_{q} Q \stackrel{V_{p} \times \operatorname{id}_{T_{q} Q}}{\longrightarrow} T_{p} P \oplus T_{q} Q \\
& T_{(p, q)} \rho_{Q} \downarrow \\
& T_{[p, q]}\left(P \times_{G} Q\right) \underset{V_{[p, q]}}{\longrightarrow} T_{[p, q]}\left(P \times_{G} Q\right)
\end{aligned} .
$$

Por outro lado, note que, para todo ponto $p \in P$ com $m=\rho(p) \in M$ e todo ponto $q \in Q$, o diagrama comutativo (2.35) induz um diagrama comutativo

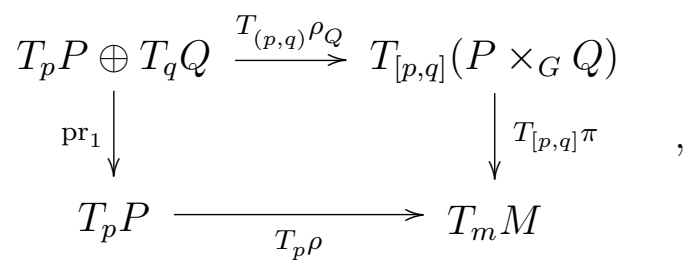

onde a aplicação $T_{(p, q)} \rho_{Q}$ é sobrejetora com núcleo

$$
\operatorname{ker} T_{(p, q)} \rho_{Q}=\left\{\left(X^{P}(p),-X^{Q}(q)\right) \mid X \in \mathfrak{g}\right\} \cong \mathfrak{g},
$$

implicando que o espaço vertical para a projeção $\pi$ de $P \times{ }_{G} Q$ sobre $M$ é

$$
V_{[p, q]}\left(P \times_{G} Q\right)=T_{(p, q)} \rho_{Q}\left(V_{p} P \oplus T_{q} Q\right) .
$$

Agora, é fácil ver que a aplicação linear $V_{[p, q]}$ que aparece no diagrama (2.134) é bem definida, mapeia $T_{[p, q]}\left(P \times_{G} Q\right)$ em $V_{[p, q]}\left(P \times_{G} Q\right)$ e é a identidade sobre $V_{[p, q]}\left(P \times_{G} Q\right)$. Finalmente, o fato de que a projeção vertical induzida $V_{[p, q]}$ depende do par $(p, q)$ apenas através da sua classe de equivalência $[p, q]$ decorre da $G$-equivariância das projeções verticais $V_{p}$ da conexão principal original.

Para chegar ao conceito da derivada covariante, recordamos que uma conexão no fibrado $P \times{ }_{G} Q$ pode também ser vista como uma seção do seu fibrado de jatos de primeira ordem $J\left(P \times_{G} Q\right)$ (como fibrado sobre $P \times_{G} Q$, não sobre $M$ ) e que uma tal seção define um isomorfismo do fibrado afim $J\left(P \times_{G} Q\right)$ com o seu fibrado vetorial de diferenças $\vec{J}\left(P \times_{G} Q\right)$ (pois impõe a escolha de uma origem em cada fibra). Desta forma, podemos concluir que uma conexão principal em $P$, representada por sua forma de conexão $A$ e através da conexão induzida no fibrado associado $P \times{ }_{G} Q$, conforme explicado acima, gera um operador $D^{A}$ de derivada covariante que atua sobre seções de $P \times_{G} Q$, levando cada tal seção $\varphi$ em uma seção $D^{A} \varphi$ do pull-back de $\vec{J}\left(P \times_{G} Q\right)$ por $\varphi$ :

$$
\varphi \in \Gamma\left(P \times_{G} Q\right) \Longrightarrow D^{A} \varphi \in \Gamma\left(\varphi^{*}\left(\vec{J}\left(P \times_{G} Q\right)\right)\right) .
$$


Também podemos considerar $D^{A}$ como um operador que atua sobre pares formados por campos vetoriais sobre $M$ e seções de $P \times_{G} Q$, levando cada tal par $(X, \varphi)$ em uma seção $D_{X}^{A} \varphi$ do pull-back de $V\left(P \times{ }_{G} Q\right)$ por $\varphi$, i.e., $D_{X}^{A} \varphi$ é um campo vetorial vertical sobre $P \times{ }_{G} Q$ ao longo de $\varphi$ :

$$
X \in \mathfrak{X}(M), \varphi \in \Gamma\left(P \times_{G} Q\right) \Longrightarrow D_{X}^{A} \varphi \in \Gamma\left(\varphi^{*}\left(V\left(P \times_{G} Q\right)\right)\right) .
$$

O fato de que o fibrado vetorial em que a derivada covariante de uma seção toma valores depende da própria seção constitui um sério obstáculo para a utilidade desta noção em fibrados gerais. A situação é substancialmente diferente para fibrados vetoriais, pois se $W$ é um espaço vetorial sobre o qual $G$ age por transformações lineares e portanto $P \times_{G} W$ é um fibrado vetorial, temos isomorfismos canônicos

$$
V\left(P \times_{G} W\right) \cong \pi^{*}\left(P \times_{G} W\right) \quad, \quad \vec{J}\left(P \times_{G} W\right) \cong \pi^{*}\left(L\left(T M, P \times_{G} W\right)\right),
$$

e portanto, vale

$$
\varphi^{*}\left(V\left(P \times_{G} W\right)\right) \cong P \times_{G} W \quad, \quad \varphi^{*}\left(\vec{J}\left(P \times_{G} W\right)\right) \cong L\left(T M, P \times_{G} W\right),
$$

para qualquer seção $\varphi$ de $P \times_{G} W$, mostrando que a derivada covariante em fibrados vetoriais é um operador linear

$$
\begin{array}{ccc}
D^{A}: \Gamma\left(P \times_{G} W\right) & \longrightarrow & \Gamma\left(L\left(T M, P \times_{G} W\right)\right) \\
\varphi & \longmapsto & D^{A} \varphi
\end{array}
$$

$\mathrm{Ou}$

$$
\begin{aligned}
& D^{A}: \mathfrak{X}(M) \times \Gamma\left(P \times_{G} W\right) \longrightarrow \Gamma\left(P \times_{G} W\right) \\
& (X, \varphi) \quad \longmapsto \quad D_{X}^{A} \varphi
\end{aligned}
$$

Voltando ao caso geral, a derivada covariante $D^{A} \varphi$ de $\varphi$ é simplesmente definida como sendo a derivada comum $d \varphi$ de $\varphi$ (que podemos identificar com o seu jato de primeira ordem $j \varphi$, ou ainda, com sua aplicação tangente $T \varphi$ ), seguido pela projeção vertical $V^{A}$ que corresponde à forma de conexão $A$ :

$$
\begin{gathered}
d \varphi(m) \in L\left(T_{m} M, T_{\varphi(m)}\left(P \times_{G} Q\right)\right) \\
\Downarrow \\
\left(D^{A} \varphi\right)(m)=V_{\varphi(m)}^{A} \circ d \varphi(m) \in L\left(T_{m} M, V_{\varphi(m)}\left(P \times_{G} Q\right)\right)
\end{gathered}
$$

Para ver que esta definição de derivada covariante é equivalente à definição usual, escolhemos um aberto $U$ de $M$ e uma seção local $\sigma$ de $P$ sobre $U$ para representar a seção $\varphi$ de $P \times_{G} Q$ 
sobre $U$ por uma função $\varphi_{\sigma} \in C^{\infty}(U, Q)$ conforme $\varphi=\left[\sigma, \varphi_{\sigma}\right]$; então para $m \in U$ com $p=\sigma(m)$ e $q=\varphi_{\sigma}(m)$, consideramos o diagrama comutativo

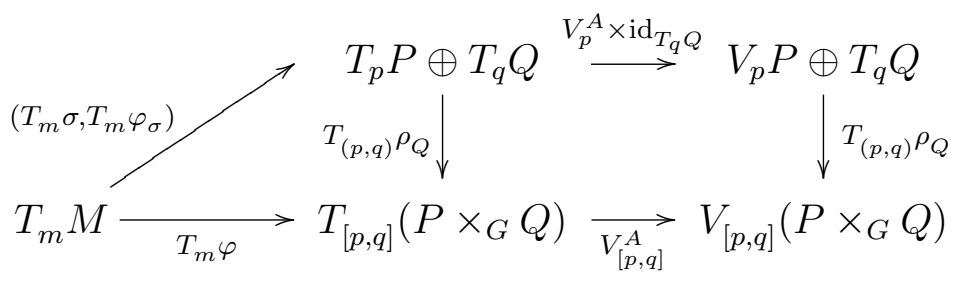

e portanto, para $m \in U$ e $u \in T_{m} M$, temos

$$
\begin{aligned}
\left(D^{A} \varphi\right)_{m}(u) & =\left(V_{[p, q]}^{A} \circ T_{m} \varphi\right)(u)=\left(V_{p, q]}^{A} \circ T_{(p, q)} \rho_{Q}\right)\left(T_{m} \sigma(u), T_{m} \varphi_{\sigma}(u)\right) \\
& =T_{(p, q)} \rho_{Q}\left(V_{p}^{A}\left(T_{m} \sigma(u)\right), T_{m} \varphi_{\sigma}(u)\right)=T_{(p, q)} \rho_{Q}\left(\left(A_{p}\left(T_{m} \sigma(u)\right)\right)^{P}(p), T_{m} \varphi_{\sigma}(u)\right) \\
& =T_{(p, q)} \rho_{Q}\left(\left(\left(\sigma^{*} A\right)_{m}(u)\right)^{P}(p), T_{m} \varphi_{\sigma}(u)\right) \\
& =T_{(p, q)} \rho_{Q}\left(0, T_{m} \varphi_{\sigma}(u)+\left(\left(\sigma^{*} A\right)_{m}(u)\right)^{Q}(q)\right)
\end{aligned}
$$

o que em termos das trivializações $P \times_{G} Q \mid U \cong U \times Q$ (veja o Teorema 2.1) e $V\left(P \times{ }_{G} Q\right) \mid U \cong U \times T Q$ induzidas por $\sigma$ corresponde à fórmula usual

$$
\left(D_{X}^{A} \varphi\right)_{\sigma}(m)=\partial_{X} \varphi_{\sigma}(m)+\left(A^{\sigma}(X)(m)\right)^{Q}\left(\varphi_{\sigma}(m)\right) .
$$

No caso de fibrados vetoriais, esta se reduz à fórmula padrão, pois se $W$ é um espaço vetorial sobre o qual $G$ age por transformações lineares, de modo que a correspondente representação de $\mathfrak{g}$ por campos fundamentais se reduz a uma representação de $\mathfrak{g}$ no sentido usual, i.e., por operadores lineares em $W$, obtemos

$$
\left(D_{X}^{A} \varphi\right)_{\sigma}=\partial_{X} \varphi_{\sigma}+A^{\sigma}(X) \cdot \varphi_{\sigma},
$$

Contemplando toda a construção do operador de derivada covariante acima apresentada, notamos que, em cada ponto $m$ da variedade base $M$, este operador depende apenas dos valores da conexão, do campo e de suas primeiras derivadas parciais neste ponto. Esta observação permite captar a essência da construção pela introdução de um homomorfismo de fibrados sobre $M$ definido por

$$
\begin{aligned}
\mathcal{D}: C P \times_{M} J\left(P \times_{G} Q\right) & \longrightarrow \vec{J}\left(P \times_{G} Q\right) \\
\left(A_{m}, j_{m} \varphi\right) & \longmapsto V_{\varphi(m)}^{A_{m}} \circ j_{m} \varphi
\end{aligned},
$$

onde, para todo ponto $p$ na fibra $P_{m}$ de $P$ sobre $m$ e todo $q \in Q, V_{[p, q]}^{A_{m}}$ é definido em termos de $V_{p}^{A_{m}}=\left(A_{m}(p)\right)^{P}(p)$ conforme a equação (2.134). Este homomorfismo será chamado de aplicação derivada covariante universal ("universal covariant derivative map") 


\subsubsection{Fibrado dos jatos do fibrado das conexões e curvatura}

Em teorias de calibre, conexões principais são variáveis dinâmicas sujeitas às suas próprias equações de movimento, provindo de um princípio variacional cuja formulação requerer considerar as suas primeiras derivadas. Como vimos anteriormente, conexões principais em um fibrado principal $P$ sobre uma variedade base $M$ podem ser consideradas como seções de um fibrado afim $C P$ sobre $M$ e portanto suas primeiras derivadas serão seções do fibrado de jatos $J(C P)$ sobre $M$. É nele que encontraremos o conceito de curvatura.

O primeiro passo consiste em aplicar o funtor $J$ ao isomorfismo (2.120) e aplicar a Proposição 2.1 para obter isomorfismos

$$
J J P \cong J\left(P \times_{M} C P\right) \cong J P \times_{M} J(C P) \cong P \times_{M} C P \times_{M} J(C P) .
$$

Então é fácil ver que a projeção $\tau_{J P}$ corresponde à projeção de $P \times_{M} C P \times_{M} J(C P)$ em $P \times{ }_{M} C P$ que é a identidade no primeiro fator, ignora o segundo e é a projeção alvo no terceiro, enquanto que a projeção $J \tau_{P}$ corresponde à projeção de $P \times_{M} C P \times_{M} J(C P)$ em $P \times{ }_{M} C P$ que é a identidade nos primeiros dois fatores e ignora o terceiro. Portanto, o subfibrado $\bar{J}^{2} P$ de $J J P$ corresponde ao subfibrado de $P \times_{M} C P \times_{M} J(C P)$ formado pelas triplas cuja segunda componente é igual à projeção alvo da terceira componente e assim temos um isomorfismo

$$
\bar{J}^{2} P \cong P \times_{M} J(C P) .
$$

Quanto à ação do grupo $G$, notamos que sob o isomorfismo (2.122), a ação induzida de $G$ em $J P$ corresponde à ação original de $G$ em $P$ multiplicada pela ação trivial de $G$ em $C P$ $(C P$ foi definido como o quociente de $J P$ pela ação de $G$ e portanto $G$ não age em $C P$ ). Portanto, sob os isomorfismos (2.146), a ação induzida de $G$ em $J J P$ corresponde à ação original de $G$ em $P$ no primeiro fator multiplicada pela ação trivial de $G$ nos demais fatores. Assim, como $P / G=M$, vem

$$
(J J P) / G \cong C P \times_{M} J(C P),
$$

e

$$
\left(\bar{J}^{2} P\right) / G \cong J(C P) .
$$

Notamos que este último isomorfismo vale não apenas como fibrados sobre $M$ mas também como fibrados sobre $C P$; veja também [22].

A principal utilidade do isomorfismo (2.149) é que podemos aplicar o Lema 2.1 para decompor o fibrado $J(C P)$ em uma "parte simétrica" e uma "parte antissimétrica", pois é fácil ver que a decomposição (2.89) (com $P$ no lugar de $E$ ) é $G$-invariante. Assim, usando ainda o isomorfismo canônico $V P \cong P \times \mathfrak{g} \cong \rho^{*}\left(P \times_{G} \mathfrak{g}\right)$ que é característico dos fibrados principais, vemos que a parte simétrica e o quociente $\left(J^{2} P\right) / G$, que é um subfibrado afim de 
$\left(\bar{J}^{2} P\right) / G$ cujo fibrado vetorial de diferenças é $\pi_{C P}^{*}\left(\bigvee^{2} T^{*} M \otimes\left(P \times_{G} \mathfrak{g}\right)\right)$, ao passo que a parte antissimétrica é o subfibrado vetorial $\pi_{C P}^{*}\left(\bigwedge^{2} T^{*} M \otimes\left(P \times_{G} \mathfrak{g}\right)\right)$ de $\left(\vec{J}^{2} P\right) / G$, e chegamos ao seguinte

Teorema 2.2 Para um fibrado principal $P$ sobre $M$, o fibrado dos jatos $J(C P)$ do fibrado das conexões $C P$ de $P$ admite uma decomposição canônica

$$
J(C P) \cong\left(J^{2} P\right) / G \times_{C P} \pi_{C P}^{*}\left(\bigwedge^{2} T^{*} M \otimes\left(P \times_{G} \mathfrak{g}\right)\right)
$$

na categoria dos fibrados afins sobre CP.

Em particular, a projeção sobre o segundo fator constitui um homomorfismo de fibrados afins sobre $C P$

$$
\mathcal{F}: J(C P) \longrightarrow \pi_{C P}^{*}\left(\bigwedge^{2} T^{*} M \otimes\left(P \times_{G} \mathfrak{g}\right)\right)
$$

que chamamos de aplicação curvatura universal ("universal curvature map") pois dada uma conexão principal qualquer em $P$, representada por uma seção $A$ de $C P$ cuja primeira derivada é representada pela seção $j A$ de $J(C P)$, a composição $F=\mathcal{F} \circ j A$ será a forma de curvatura desta seção.

\section{Estrutura local}

Para construir trivializações locais explícitas do fibrado $J(C P)$, definidas a partir de uma seção $\sigma$ do fibrado principal $P$ sobre um aberto $U$ da variedade base $M$, em conjunto com uma carta $x$ para $U$, como sempre, aplicamos o funtor $J$ à trivialização $C \Phi_{\sigma}^{x}$ de $C P$ sobre $U$ definida na equação (2.125), conforme a regra estabelecida nas equações (2.49)-(2.51), e usamos o isomorfismo

$$
J_{0}^{1}\left(\mathbb{R}^{n}, L\left(\mathbb{R}^{n}, \mathfrak{g}\right)\right) \cong L\left(\mathbb{R}^{n}, \mathfrak{g}\right) \times L^{2}\left(\mathbb{R}^{n}, \mathfrak{g}\right)
$$

(obtido da equação (2.48) substituindo a variedade $Q$ pelo espaço afim $L\left(\mathbb{R}^{n}, \mathfrak{g}\right)$ ) para obter a seguinte trivialização de $J(C P)$ sobre $U$,

$$
\begin{array}{cccc}
J(C \Phi)_{\sigma}^{x}:\left.\quad J(C P)\right|_{U} & \longrightarrow & U \times L\left(\mathbb{R}^{n}, \mathfrak{g}\right) \times L^{2}\left(\mathbb{R}^{n}, \mathfrak{g}\right) \\
j_{m} \Gamma^{A} \cong j_{m} A & \longmapsto\left(m, A_{m}^{\sigma} \circ D_{x^{-1}(m)} x^{-1}, B_{m}^{\sigma} \circ D_{x^{-1}(m)}^{2} x^{-1}\right)
\end{array}
$$

onde $A_{m}^{\sigma} \in L\left(T_{m} M, \mathfrak{g}\right)$ e $B_{m}^{\sigma} \in L^{2}\left(T_{m} M, \mathfrak{g}\right)$ são construídos da seguinte forma: IDENTIFICAR PARTE SIMÉTRICA E PARTE ANTISIMÉTRICA PARA $B_{m}^{\sigma}$ ! 


\section{$2.4 \quad$ Fibrado dos cojatos}

O fibrado de cojatos $J^{\circledast} E$ de $E$ é obtido tomando o dual afim "torcido" do fibrado dos jatos $J E$, isto é, o fibrado vetorial sobre $E$ cuja fibra em $e$ é formada pelas aplicações afins

$$
z_{e}: J_{e} E \longrightarrow \bigwedge^{n} T_{m}^{*} M \quad \text { onde } m=\pi(e)
$$

Este fibrado carrega uma n-forma canônica que localmente, em coordenadas de fibrado $\left(x_{\mu}, q^{i}, p_{i}^{\mu}, p\right)$, assume a forma

$$
\theta=p_{i}^{\mu} d q^{i} \wedge d^{n} x_{\mu}+p d^{n} x
$$

A forma multissimplética é a derivada exterior $\omega=-d \theta$ dessa n-forma. 


\section{Simetrias globais e locais na teoria dos campos}

Neste capítulo, queremos abordar a questão central da presente tese, a saber, como formular os diversos conceitos de simetria que aparecem na teoria (clássica) dos campos num âmbito geométrico geral, onde o espaço-tempo é uma variedade lorentziana $M$ geral e os campos são seções de algum fibrado $E$ sobre $M$ : tipicamente, este fibrado será um fibrado associado a um fibrado principal $P$ ou o fibrado $C P$ das conexões sobre $P$ ou uma combinação destes dois. Essa questão se coloca tanto para simetrias espaço-temporais como para simetrias internas e ainda para simetrias compostas por estes dois tipos, envolvendo uma passagem altamente não-trivial onde simetrias globais "rígidas" são substituídas por simetrias locais "flexíveis".

No caso de simetrias espaço-temporais, o procedimento constitui o cerne da transição da relatividade restrita para a relatividade geral. A relatividade restrita descreve a física no espaço-tempo plano de Minkowski e é caracterizada por sua invariância sob o grupo de Lorentz (ou de Poincaré, quando incluímos as translações) - uma simetria global, rígida. A transição para a relatividade geral é efetuada por uma imensa generalização desta simetria, substituindo o espaço-tempo de Minkowski por uma variedade lorentziana $M$ geral e postulando a invariância das leis da física sob transformações arbitrárias de coordenadas (locais), ou difeomorfismos, conforme o princípio de covariância geral.

Nas diversas formulações do princípio de covariância geral, aparecem duas noções de transformações entre diferentes sistemas de coordenadas ou referenciais utilizados na descrição de um determinado sistema físico, refletindo dois pontos de vista diferentes e de certa forma complementares, conhecidos como o "passivo" e 
o "ativo". ${ }^{1}$ Por um lado, do ponto de vista passivo, uma transformação de coordenadas descreve o efeito de uma mudança do referencial empregado para parametrizar os pontos do espaço-tempo, que são fixos. Por outro lado, do ponto de vista ativo, uma transformação de coordenadas descreve o efeito de uma transformação que move os pontos do espaço-tempo, em relação a um referencial fixo. Isso implica que sob o ponto de vista passivo, transformações de coordenadas são necessariamente locais, pois estão restritas ao domínio de validade do referencial ou sistema de coordenadas inicial, enquanto que sob o ponto de vista ativo, elas admitem uma interpretação global, como difeomorfismos do espaço-tempo subjacente.

De qualquer modo, esta invariância configura uma simetria local, flexível, sendo que as simetrias globais anteriores podem ser recuperadas considerando o grupo "global" I $(M)$ das isometrias de $M$, que é um subgrupo do grupo "local" Diff( $M)$ dos difeomorfismos de $M$.

No caso de simetrias internas, o procedimento é inteiramente análogo e constitui a idéia básica de como "calibrar uma simetria", desenvolvida por H. Weyl na sua tentativa de unificar eletromagnetismo com gravitação. Aqui, temos como ponto de partida uma teoria clássica de campos onde todos os diferentes campos são reunidos em um único campo a valores num espaço vetorial interno e sujeitos a uma simetria rígida descrita pela invariância em relação a uma representação de um grupo interno $G$ sobre este espaço vetorial. Mais uma vez, a transição para uma teoria de calibre é efetuada por uma imensa generalização desta simetria, substituindo o produto cartesiano do espaço vetorial interno com o espaço-tempo $M$ por um fibrado $E$ sobre $M$ e postulando a invariância das leis da física sob mudanças arbitrárias de trivializações (locais), ou automorfismos estritos, conforme o princípio de invariância de calibre.

Novamente, nas diversas formulações do princípio de invariância de calibre, aparecem duas noções de transformações de calibre que refletem dois pontos de vista diferentes e de certa forma complementares, conhecidos como o "passivo" e o "ativo". Por um lado, do ponto de vista passivo, uma transformação de calibre descreve o efeito de uma mudança de trivialização equivariante no fibrado principal $P$ pertinente. Como, em fibrados principais, trivializações (locais) equivariantes correspondem a seções (locais), podemos definir a passagem de uma

\footnotetext{
${ }^{1}$ Note que a palavra "referencial" pode ser utilizada em vários sentidos diferentes, dependendo do contexto. Na mecânica Newtoniana assim como na relatividade restrita, existe um conjunto de referenciais privilegiados, chamados de referenciais inerciais, sendo que quaisquer dois referenciais inerciais podem ser transformados um no outro por uma única transformação de Galilei, no caso da mecânica Newtoniana, ou de Lorentz, no caso da relatividade restrita. No âmbito da relatividade geral, a palavra "referencial" significa "sistema de coordenadas locais para o espaço-tempo" (normais ou não), e é neste sentido que utilizaremos o termo aqui, embora ainda ser possível considerar referenciais mais gerais, chamados de não-holônomos.
} 
trivialização equivariante de $P$ dada por uma seção $\sigma$ para outra trivialização equivariante de $P$ dada por outra seção $\tau$, todas sobre um determinado aberto $U$ de $M$, como determinada por uma função $g \in C^{\infty}(U, G)$, segundo a fórmula

$$
\tau(m)=\sigma(m) \cdot g(m) \quad \text { para } m \in U .
$$

Tendo em vista que as trivializações equivariantes de $P$ sobre $U$ induzidas por $\sigma$ e por $\tau$ são dadas, respectivamente, pelos isomorfismos

$$
\begin{aligned}
U \times G & \left.\longrightarrow P\right|_{U} \\
(m, g) & \longmapsto \sigma(m) \cdot g
\end{aligned},
$$

$\mathrm{e}$

$$
\begin{aligned}
U \times G & \left.\longrightarrow P\right|_{U} \\
(m, g) & \longmapsto \tau(m) \cdot g
\end{aligned},
$$

esta passagem entre trivializações de $P$ sobre $U$ é explicitamente dada pela fórmula

$$
\begin{array}{ccc}
U \times G & \longrightarrow U \times G \\
(m, g) & \longmapsto & (m, g(m) g)
\end{array}
$$

Uma lei de transformação análoga vale em qualquer fibrado associado $P \times_{G} Q$, pois as trivializações de $P \times_{G} Q$ sobre $U$ induzidas por $\sigma$ e por $\tau$ são dadas, respectivamente, pelos isomorfismos

$$
\begin{aligned}
U \times Q & \left.\longrightarrow\left(P \times_{G} Q\right)\right|_{U} \\
(m, q) & \longmapsto[\sigma(m), q]
\end{aligned}
$$

$\mathrm{e}$

$$
\begin{aligned}
& U \times\left. Q \longrightarrow\left(P \times_{G} Q\right)\right|_{U} \\
& (m, q) \longmapsto[\tau(m), q] \quad
\end{aligned}
$$

levando à mudança de trivialização dada por

$$
\begin{gathered}
U \times Q \quad \longrightarrow \quad U \times Q \\
(m, q) \longmapsto(m, g(m) \cdot q)
\end{gathered}
$$

Por outro lado, do ponto de vista ativo, uma transformação de calibre descreve o efeito de uma transformação que move os pontos do fibrado principal $P$, em relação a uma trivialização fixa. Explicitamente, transformações de calibre podem ser definidas como automorfismos (mais exatamente, automorfismos estritos) do fibrado principal $P$ sobre $M$, ou seja, como difeomorfismos $f: P \rightarrow P$ de $P$ que preservam as fibras de $P$ sobre $M$ e são equivariantes sob a ação de $G$ :

$$
f(p \cdot g)=f(p) \cdot g \quad \text { para } p \in P, g \in G .
$$


Tal automorfismo $f$ de $P$ induz um automorfismo $f_{Q}: P \times_{G} Q \rightarrow P \times_{G} Q$ de qualquer fibrado associado $P \times_{G} Q$, conforme

$$
f_{Q}[p, q]=[f(p), q] \quad \text { para } p \in P, q \in Q .
$$

Fixando uma trivialização equivariante de $P$ sobre um determinado aberto $U$ de $M$ dada por uma seção $\sigma$ de $P$ sobre $U$, podemos representar o automorfismo $f$ por uma função $f_{\sigma} \in C^{\infty}(U, G)$ conforme

$$
f(\sigma(m) \cdot g)=\sigma(m) \cdot\left(f_{\sigma}(m) g\right) \quad \text { para } m \in U, g \in G,
$$

ou usando o isomorfismo (3.2)

$$
f(m, g)=\left(m, f_{\sigma}(m) g\right) \quad \text { para } m \in U, g \in G .
$$

Note que passando para outra trivialização equivariante de $P$ sobre o mesmo aberto $U$ de $M$, que corresponde a uma seção $\tau$ de $P$ sobre $U$ relacionada com a seção $\sigma$ de $P$ sobre $U$ conforme a eq. (3.1), obtemos

$$
f_{\tau}(m)=g(m)^{-1} f_{\sigma}(m) g(m) \quad \text { para } m \in U .
$$

Assim, vemos a partir das equações (3.4) e (3.10) ou (3.11) que, localmente, uma transformação de calibre aparece, tanto do ponto de vista passivo como do ponto de vista ativo, como multiplicação à esquerda por uma função a valores em $G$. No entanto, sob o ponto de vista passivo transformações de calibre são necessariamente locais, pois estão restritas ao domínio de validade da trivialização inicial, enquanto que sob o ponto de vista ativo, elas admitem uma interpretação global, como automorfismos estritos do fibrado principal subjacente.

Concluímos, como antes, que esta invariância configura uma simetria local, flexível, mas ao contrário da situação encontrada acima, as simetrias globais anteriores não podem ser recuperadas como um subgrupo do grupo das simetrias locais, pois o grupo $\operatorname{Gau}(P)$ dos automorfismos estritos do fibrado principal $P^{2}$ não contém nenhum subgrupo natural isomorfo a $G$.

Observação 3.1 Existem duas outras implementações matemáticas da noção global de transformação de calibre que são equivalentes à anterior. A primeira é baseada na observação de que um automorfismo estrito $f$ de $P$ como fibrado principal sobre $M$ corresponde biunivocamente a uma função $\tilde{f}$ sobre $P$ a valores em $G$ que é equivariante em relação à ação de $G$ sobre $P$ e à ação de $G$ sobre $G$ mesmo por conjugação, ou seja, que satisfaz

$$
\tilde{f}(p \cdot g)=g^{-1} \tilde{f}(p) g \quad \text { para } p \in P, g \in G .
$$

\footnotetext{
${ }^{2} \mathrm{O}$ símbolo "Gau" é uma abreviação da expressão "gauge group".
} 
Explicitamente, a correspondência é dada por

$$
f(p)=p \cdot \tilde{f}(p) \quad \text { para } p \in P, g \in G .
$$

A segunda é baseada na observação de que uma função $\tilde{f}$ de $P$ a valores em $G$ que é equivariante no sentido acima descrito corresponde biunivocamente a uma seção $\hat{f}$ do fibrado associado $P \times_{G} G$ definido usando a ação de $G$ (como grupo estrutural de $P$ ) em $G$ (como fibra típica do fibrado associado) por conjugação. Explicitamente, a correspondência é dada por

$$
\hat{f}(m)=[p, \tilde{f}(p)] \quad \text { para } m \in M, p \in P_{m}, g \in G .
$$

Assim, obtemos isomorfismos canônicos

$$
\operatorname{Gau}(P) \cong C_{E}^{\infty}(P, G) \cong \Gamma\left(P \times_{G} G\right) .
$$

Isso mostra, novamente, que, globalmente, transformações de calibre são de certa forma funções sobre o espaço-tempo, mas não são simplesmente funções sobre $M$ a valores em $G$ e sim seções de um fibrado sobre $M$ com fibra típica $G$ mas com funções de transição nãotriviais, como evidenciado pela lei de transformação (3.12).

Finalmente, podemos unificar simetrias locais espaço-temporais e simetrias locais internas considerando o grupo Aut $(P)$ de todos os automorfismos do fibrado principal $P$, que contém o grupo $\operatorname{Gau}(P)$ como subgrupo normal e admite o grupo Diff $(M)$ como grupo quociente, ou seja, temos a seguinte sequência exata de grupos:

$$
1 \longrightarrow \operatorname{Gau}(P) \longrightarrow \operatorname{Aut}(P) \longrightarrow \operatorname{Diff}(M) \longrightarrow 1 \text {. }
$$

Novamente, enfrentamos o problema de que as simetrias globais não aparecem naturalmente como simetrias locais especiais, pois o grupo $\operatorname{Aut}(P)$ não contém nenhum subgrupo natural isomorfo a $\mathrm{I}(M)$ ou a $G$.

Cabe notar que o grupo $\operatorname{Aut}(P)$ é enorme e portanto é difícil encontrar funcionais ação com um grupo de simetria tão grande. De fato, modelos com este grupo de simetria têm recebido um nome particular: são chamados de "teorias de campos topológicas" (topological field theories). Os exemplos mais conhecido são as teorias de Chern-Simons, com $\operatorname{dim} M=3$.

Como já foi mencionado anteriormente, a desvantagem fundamental da abordagem que acabamos de descrever consiste no fato de que grupos tais como $\operatorname{Diff}(M), \operatorname{Gau}(P)$ e $\operatorname{Aut}(P)$ são objetos de dimensão infinita e portanto difíceis de se manusear, já na teoria clássica. $\mathrm{Na}$ quantização, estes obstáculos se potenciam e, com as técnicas matemáticas atualmente disponíveis, parecem insuperáveis.

Felizmente, como veremos neste capítulo, invariância de uma teoria clássica de campos sob transformações de calibre pode ser formalizada de uma outra maneira: em vez de 
invariância do funcional ação sob a ação do grupo de calibre $\operatorname{Gau}(P)$, postulamos invariância da lagrangiana sob a ação de um certo fibrado de transformações de calibre, a ser introduzido logo adiante. Ademais, a nossa abordagem providencia um sentido matematicamente rigoroso, no âmbito geométrico mais geral possível, para o procedimento de "calibrar uma simetria", por meio do "acoplamento mínimo" a um campo de calibre.

\subsection{Fibrados de grupos de Lie}

Como já foi explicado, porém sem maiores detalhes, na Seção 2.1.5, fibrados de grupos de Lie e de álgebras de Lie são simplesmente fibrados com uma estrutura, respectivamente, de grupo de Lie e de álgebra de Lie nas fibras que é localmente constante.

Definição 3.1 Um fibrado de grupos de Lie é um fibrado $\bar{G}$ sobre uma variedade base $M$ que tem como fibra típica um grupo de Lie $G$ e que é munido de um homomorfismo estrito de fibrados sobre $M$

$$
\begin{aligned}
\bar{G} \times_{M} \bar{G} & \longrightarrow \bar{G} \\
\left(g_{1}, g_{2}\right) & \longmapsto g_{1} g_{2}
\end{aligned}
$$

chamado produto ou multiplicação, de uma seção global 1 de $\bar{G}$ chamada unidade e de um homomorfismo estrito de fibrados sobre $M$

$$
\begin{aligned}
\bar{G} & \longrightarrow \bar{G} \\
g & \longmapsto g^{-1}
\end{aligned}
$$

chamado inversão que definem uma estrutura de grupo de Lie em cada fibra de $\bar{G}$ a qual é localmente constante, conforme explicado na Seção 2.1.5.

Definição 3.2 Um fibrado de álgebras de Lie é um fibrado vetorial $\overline{\mathfrak{g}}$ sobre uma variedade base $M$ que tem como fibra típica uma álgebra de Lie $\mathfrak{g}$ e que é munido de um homomorfismo estrito de fibrados sobre $M$

$$
\begin{array}{rlc}
\overline{\mathfrak{g}} \times_{M} \overline{\mathfrak{g}} & \longrightarrow & \overline{\mathfrak{g}} \\
\left(X_{1}, X_{2}\right) & \longmapsto & {\left[X_{1}, X_{2}\right]}
\end{array}
$$

chamado de comutador que define uma estrutura de álgebra de Lie em cada fibra de $\overline{\mathfrak{g}}$ a qual é localmente constante, conforme explicado na Seção 2.1.5.

Recordamos que o requerimento de que estas estruturas sejam localmente constantes significa que, para cada ponto de $M$, deve existir uma trivialização $\Phi$ do fibrado em questão sobre alguma vizinhança aberta $U$ deste ponto tal que para todo $m \in U$, o difeomorfismo 
$\Phi_{m}: \bar{G}_{m} \rightarrow G$, no caso de um fibrado de grupos de Lie, é um isomorfismo de grupos de Lie, levando o produto, a unidade e a inversão em $\bar{G}_{m}$ para o produto, a unidade e a inversão em $G$, e o isomorfismo linear $\dot{\Phi}_{m}: \overline{\mathfrak{g}}_{m} \rightarrow \mathfrak{g}$, no caso de um fibrado de álgebras de Lie, é um isomorfismo de álgebras de Lie, levando o comutador em $\overline{\mathfrak{g}}_{m}$ para o comutador em $\mathfrak{g}$. Como já foi mencionado na Seção 2.1.5, tais trivializações locais serão chamadas de compatíveis.

Notamos também que a função de transição entre duas trivializações locais compatíveis toma valores no grupo dos automorfismos da fibra típica, e como o grupo $\operatorname{Aut}(G)$ dos automorfismos de um grupo de Lie $G$ e o grupo $\operatorname{Aut}(\mathfrak{g})$ dos automorfismos de uma álgebra de Lie $\mathfrak{g}$ são grupos de Lie, concluímos que todo fibrado de grupos de Lie é um fibrado com grupo estrutural Aut $(G)$ e todo fibrado de álgebras de Lie é um fibrado com grupo estrutural Aut(g). Contudo, é perfeitamente concebível que o grupo estrutural destes fibrados seja menor, isto é, um subgrupo do grupo dos automorfismos da fibra típica: por exemplo, no caso de um fibrado trivial, ele pode ser reduzido ao subgrupo trivial $\{1\}$. De modo geral, podemos admitir a possibilidade de que o grupo estrutural seja um outro grupo de Lie, que a seguir denotaremos por $\Gamma$ : tudo que é preciso é que este aja sobre a fibra típica por automorfismos.

Observação 3.2 Estas observações sugerem a seguinte abordagem alternativa, que de um ponto de partida diferente chega ao mesmo resultado. Seja $P$ um fibrado principal sobre uma variedade base $M$ com projeção $\rho: P \rightarrow M$ e grupo estrutural $\Gamma$. Seja $G$ um grupo de Lie munido de uma ação de $\Gamma$ à esquerda

$$
\begin{aligned}
\Gamma \times G & \longrightarrow G \\
(\gamma, g) & \longmapsto \gamma \cdot g
\end{aligned}
$$

por automorfismos, i.e., tal que

$$
\gamma \cdot\left(g_{1} g_{2}\right)=\left(\gamma \cdot g_{1}\right)\left(\gamma \cdot g_{2}\right) \quad \text { para } \gamma \in \Gamma, g_{1}, g_{2} \in G \text {. }
$$

Então o fibrado associado $P \times_{\Gamma} G$ é um fibrado de grupos de Lie, onde o produto e a inversão são explicitamente dados por

$$
\left[p, g_{1}\right]\left[p, g_{2}\right]=\left[p, g_{1} g_{2}\right] \quad \text { para } p \in P, g_{1}, g_{2} \in G
$$

$\mathrm{e}$

$$
[p, g]^{-1}=\left[p, g^{-1}\right] \quad \text { para } p \in P, g \in G .
$$

Da mesma forma, seja $\mathfrak{g}$ uma álgebra de Lie munida de uma ação de $\Gamma$ à esquerda

$$
\begin{aligned}
\Gamma \times \mathfrak{g} & \longrightarrow \mathfrak{g} \\
(\gamma, X) & \longmapsto \gamma \cdot X
\end{aligned}
$$


por automorfismos, i.e., tal que $\gamma \cdot X$ é linear em $X$ e

$$
\gamma \cdot\left[X_{1}, X_{2}\right]=\left[\gamma \cdot X_{1}, \gamma \cdot X_{2}\right] \quad \text { para } \gamma \in \Gamma, X_{1}, X_{2} \in \mathfrak{g} .
$$

Então o fibrado associado $P \times_{\Gamma} \mathfrak{g}$ é um fibrado de álgebras de Lie, onde o comutador é explicitamente dado por

$$
\left[\left[p, X_{1}\right],\left[p, X_{2}\right]\right]=\left[p,\left[X_{1}, X_{2}\right]\right] \quad \text { para } p \in P, X_{1}, X_{2} \in \mathfrak{g} .
$$

Neste caso, naturalmente, a situação mais importante ocorre quando temos $\Gamma=G$, sendo que $G$ age sobre si mesmo por conjugação e sobre sua própria álgebra de Lie $\mathfrak{g}$ pela representação adjunta.

Definição 3.3 Seja $P$ um fibrado principal sobre uma variedade base $M$ com projeção $\rho: P \rightarrow M$ e grupo estrutural $G$. O fibrado de grupos de Lie $P \times_{G} G$, onde $G$ age sobre si mesmo por conjugação, e o fibrado de álgebras de Lie $P \times_{G} \mathfrak{g}$, onde $G$ age sobre sua álgebra de Lie pela representação adjunta, são chamados os fibrados de transformações de calibre (globais e infinitesimais, respectivamente) de $P$.

Ressaltamos, mais uma vez, que é vantajoso admitir a possibilidade de desvincular a escolha dos dois grupos de Lie envolvidos na definição de um fibrado de grupos de Lie: o grupo estrutural $\Gamma$ e a fibra típica $G$, pois na grande maioria das aplicações, o primeiro será um subgrupo do segundo que pode ser muito menor: por exemplo, no caso de fibrados triviais, $\Gamma$ será trivial $(=\{1\})$ mas, obviamente, $G$ não será trivial. E mesmo quando são iguais, como na definição anterior, a distinção entre eles ainda é útil, pois desempenham papéis diferentes na teoria.

A relação natural entre grupos de Lie e álgebras de Lie é facilmente estendida a fibrados de grupos e álgebras de Lie. A observação básica é que cada fibrado de grupos de Lie $\bar{G}$, com fibra típica $G$, induz naturalmente um fibrado de álgebras de Lie $\overline{\mathfrak{g}}$, com fibra típica $\mathfrak{g}$, onde $\mathfrak{g}$ é a álgebra de Lie de $G$ : basta escolher, para todo ponto $m$ da variedade base $M$, $\overline{\mathfrak{g}}_{m}$ como sendo a álgebra de Lie de $\bar{G}_{m}$ e definir, para cada trivialização local compatível $\Phi: \bar{G} \mid U \rightarrow U \times G$ de $\bar{G}$, uma trivialização local compatível $\dot{\Phi}: \overline{\mathfrak{g}} \mid U \rightarrow U \times \mathfrak{g}$ de $\overline{\mathfrak{g}}$ supondo que para todo ponto $m$ de $U$, o isomorfismo de álgebras de Lie $\dot{\Phi}_{m}: \overline{\mathfrak{g}}_{m} \rightarrow \mathfrak{g}$ é a derivada em 1 do isomorfismo de grupos de Lie $\Phi_{m}: \bar{G}_{m} \rightarrow G$. Logo, para todo ponto $m$ de $U$, o diagrama

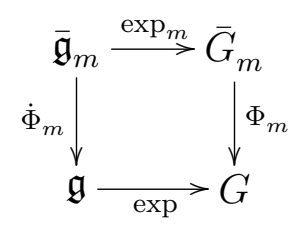


comuta, o que permite reunir todos os $\exp _{m}$ dentro de um homomorfismo estrito de fibrados sobre $M$

$$
\begin{array}{ccc}
\overline{\mathfrak{g}} & \longrightarrow & \bar{G} \\
X & \longmapsto & \exp (X)
\end{array}
$$

chamado de exponencial, que é localmente constante pois, em trivializações locais compatíveis construídas como acima, é representado pela exponencial usual exp $: \mathfrak{g} \rightarrow G$, independentemente de $m$. Do ponto de vista de fibrados associados a um fibrado principal $P$ sobre $M$ com grupo estrutural $\Gamma$, podemos entender esta construção se observamos que cada ação de $\Gamma$ sobre um grupo de Lie $G$ por automorfismos de $G$ induz uma ação de $\Gamma$ sobre a sua álgebra de Lie $\mathfrak{g}$ por automorfismos de $\mathfrak{g}$ e tal que o homomorfismo canônico

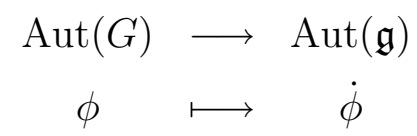

definido por

$\mathrm{Ou}$

$$
\dot{\phi}\left(\left.\frac{d}{d t} g(t)\right|_{t=0}\right)=\left.\frac{d}{d t} \phi(g(t))\right|_{t=0}
$$

$$
\exp (t \dot{\phi}(X))=\phi(\exp (t X))
$$

torna-se $\Gamma$-equivariante. Assim, o fibrado de álgebras de Lie induzido pelo fibrado de grupos de Lie $P \times_{\Gamma} G$ associado a $P$ é naturalmente identificado com o fibrado de álgebras de Lie $P \times_{\Gamma} \mathfrak{g}$ associado a $P$, e de tal forma que a aplicação exponencial (3.28) é dada em termos da aplicação exponencial usual $\exp : \mathfrak{g} \rightarrow G$ pela fórmula

$$
\exp [p, X]=[p, \exp (X)] \quad \text { para } p \in P, X \in \mathfrak{g} .
$$

\subsection{Ações de fibrados de grupos de Lie}

O conceito de uma ação de um fibrado de grupos de Lie sobre um fibrado é mais facilmente obtido como generalização do conceito do produto por fibras dentro de um fibrado de grupos de Lie, justamente como o conceito de uma ação de um grupo de Lie sobre uma variedade pode ser considerado como generalização do conceito do produto dentro de um grupo de Lie.

Definição 3.4 Uma ação de um fibrado de grupos de Lie $\bar{G}$ em um fibrado E, ambos sobre a mesma variedade base $M$, é um homomorfismo estrito de fibrados sobre $M$

$$
\begin{aligned}
& \bar{G} \times{ }_{M} E \longrightarrow E \\
& (g, e) \longmapsto g \cdot e
\end{aligned}
$$

que define uma ação de cada fibra de $\bar{G}$ sobre a fibra de E com mesmo ponto base a qual é localmente constante, conforme explicado na Seção 2.1.5. 
Novamente, o requerimento de que a ação seja localmente constante significa que devem existir uma ação

$$
\begin{array}{cc}
G \times Q & \longrightarrow Q \\
(g, q) & \longmapsto g \cdot q
\end{array}
$$

da fibra típica $G$ de $\bar{G}$ na fibra típica $Q$ de $E$ assim como, para cada ponto de $M$, uma trivialização do fibrado $\bar{G}$ compatível com sua estrutura de fibrado de grupos de Lie e uma trivialização do fibrado $E$, ambas definidas sobre alguma vizinhança aberta $U$ deste ponto, tal que para todo $m \in U$, o isomorfismo entre a fibra $\bar{G}_{m}$ e a fibra típica $G$ de $\bar{G}$ e o isomorfismo entre a fibra $E_{m}$ e a fibra típica $Q$ de $E$ induzidos por estas trivializações locais levam a ação de $\bar{G}_{m}$ em $E_{m}$ dada pela ação (3.30) para a ação (3.31) de $G$ em $Q$. Mais uma vez, tais trivializações locais serão chamadas de compatíveis.

Observação 3.3 A implementação da noção de uma ação de um fibrado de grupos de Lie em um fibrado no âmbito de fibrados com grupo estrutural, que podem ser escritos como fibrados associados a algum fibrado principal, leva a uma abordagem alternativa, que é a seguinte. Seja $P$ um fibrado principal sobre uma variedade base $M$ com projeção $\rho: P \rightarrow M$ e grupo estrutural $\Gamma$. Seja $G$ um grupo de Lie munido de uma ação

$$
\begin{gathered}
\Gamma \times G \quad \longrightarrow G \\
(\gamma, g) \quad \longmapsto \gamma \cdot g
\end{gathered}
$$

de $\Gamma$ por automorfismos, i.e., tal que

$$
\gamma \cdot\left(g_{1} g_{2}\right)=\left(\gamma \cdot g_{1}\right)\left(\gamma \cdot g_{2}\right) \quad \text { para } \gamma \in \Gamma, g_{1}, g_{2} \in G,
$$

como antes (veja as equações (3.21) e (3.22)), e seja $Q$ uma variedade munida de uma ação

$$
\begin{aligned}
\Gamma \times Q & \longrightarrow Q \\
(\gamma, q) & \longmapsto \gamma \cdot q
\end{aligned}
$$

de $\Gamma$, de modo que podemos introduzir os fibrados associados $P \times_{\Gamma} G$ e $P \times_{\Gamma} Q$, sendo o primeiro um fibrado de grupos de Lie. Então dada uma ação

$$
\begin{array}{ccc}
G \times Q & \longrightarrow Q \\
(g, q) & \longmapsto g \cdot q
\end{array}
$$

de $G$ em $Q$, como antes (veja a equação (3.31)) que é $\Gamma$-equivariante, i.e., satisfaz

$$
\gamma \cdot(g \cdot q)=(\gamma \cdot g) \cdot(\gamma \cdot q) \quad \text { para } \gamma \in \Gamma, g \in G, q \in Q,
$$

obtemos uma ação

$$
\begin{aligned}
& \left(P \times_{\Gamma} G\right) \times_{M}\left(P \times_{\Gamma} Q\right) \longrightarrow P \times_{\Gamma} Q \\
& ([p, g],[p, q]) \quad \longmapsto[p, g \cdot q]
\end{aligned}
$$


do fibrado de grupos de Lie $P \times_{\Gamma} G$ sobre $M$ no fibrado $P \times_{\Gamma} Q$ sobre $M$, no sentido da definição acima.

Em particular, se $P$ é um fibrado principal sobre uma variedade base $M$ com projeção $\rho: P \rightarrow M$ e grupo estrutural $G$ e se $Q$ é uma variedade munida de uma ação de $G$, então obtemos uma ação do fibrado $P \times_{G} G$ de transformações de calibre de $P$ sobre o fibrado associado $P \times_{G} Q$, dada por

$$
\begin{aligned}
& \left(P \times_{G} G\right) \times_{M}\left(P \times_{G} Q\right) \longrightarrow P \times_{G} Q \\
& ([p, g],[p, q]) \quad \longmapsto \quad[p, g \cdot q]
\end{aligned}
$$

Como veremos logo adiante, esta construção serve como ponto de partida para a descrição de simetrias em teorias de calibre.

Passando ao nível infinitesimal, notamos que, do mesmo modo que uma ação de um grupo de Lie $G$ em uma variedade $Q$ induz uma "(anti-)representação da sua álgebra de Lie $\mathfrak{g}$ por campos vetoriais sobre $Q$ ", i.e., um anti-homomorfismo

$$
\begin{gathered}
\mathfrak{g} \longrightarrow \mathfrak{X}(Q) \\
X \longmapsto X_{Q}
\end{gathered}
$$

de $\mathfrak{g}$ tomando valores em $\mathfrak{X}(Q)$, a álgebra de Lie dos campos vetoriais sobre $Q$, uma ação de um fibrado de grupos de Lie $\bar{G}$ sobre $M$ em um fibrado $E$ sobre $M$ também induz uma "(anti-) representação do seu fibrado de álgebras de Lie $\overline{\mathfrak{g}}$ por campos vetoriais verticais sobre $E$ ", i.e., um anti-homomorfismo

$$
\begin{aligned}
& \overline{\mathfrak{g}} \longrightarrow \overline{\mathfrak{V}}(E) \\
& X \longmapsto X_{E}
\end{aligned}
$$

de $\overline{\mathfrak{g}}$ tomando valores em $\overline{\mathfrak{V}}(E)$, que formalmente é um fibrado de álgebras de Lie sobre $M$ definido por

$$
\overline{\mathfrak{V}}(E)=\bigcup_{m \in M} \mathfrak{X}\left(E_{m}\right)
$$

e cujas seções constituem a álgebra de Lie $\mathfrak{X}^{V}(E)$ dos campos vetoriais verticais sobre $E$. Explicitamente, estes campos fundamentais são definidos por

$$
X_{Q}(q)=\left.\frac{d}{d t}(\exp (t X) \cdot q)\right|_{t=0} \quad \text { para } X \in \mathfrak{g}, q \in Q
$$

no primeiro caso, e por

$$
X_{E}(e)=\left.\frac{d}{d t}(\exp (t X) \cdot e)\right|_{t=0} \quad \text { para } m \in M, X \in \overline{\mathfrak{g}}_{m}, e \in E_{m}
$$


no segundo caso. A propriedade algébrica das aplicações (3.39) e (3.40) de serem antihomomorfismos em relação ao comutador usual (no lado esquerdo) e ao colchete de Lie (no lado direito) é contida nas fórmulas

$$
[X, Y]_{Q}=-\left[X_{Q}, Y_{Q}\right] \quad \text { para } X, Y \in \mathfrak{g}
$$

no primeiro caso e

$$
[X, Y]_{E}=-\left[X_{E}, Y_{E}\right] \quad \text { para } X, Y \in \overline{\mathfrak{g}}
$$

no segundo caso, enquanto que, como sempre acontece em situações envolvendo objetos de dimensão infinita, a expressão de sua diferenciabilidade requer uma mudança de enfoque que consiste em reinterpretar a aplicação (3.39) como um homomorfismo

$$
\begin{gathered}
Q \times \mathfrak{g} \quad \longrightarrow \quad T Q \\
(q, X) \longmapsto X_{Q}(q)
\end{gathered}
$$

de fibrados vetoriais sobre $Q$, no primeiro caso, e a aplicação (3.40) como um homomorfismo

$$
\begin{array}{ccc}
\pi^{*} \overline{\mathfrak{g}} & \longrightarrow V E \\
(e, X) & \longmapsto & X_{E}(e)
\end{array}
$$

de fibrados vetoriais sobre $E$, no segundo caso.

Observação 3.4 Na situação estudada na observação anterior, onde todos os fibrados envolvidos são fibrados associados, $\bar{G}=P \times_{\Gamma} G, \overline{\mathfrak{g}}=P \times_{\Gamma} \mathfrak{g}$ e $E=P \times_{\Gamma} Q$, podemos usar as ações induzidas de $\Gamma$ em $T Q$ e em $\mathfrak{X}(Q)$ para identificar o fibrado vertical $V E$ com o fibrado associado $P \times_{\Gamma} T Q$ e o fibrado $\overline{\mathfrak{V}}(E)$ com o fibrado associado $P \times_{\Gamma} \mathfrak{X}(Q)$ e reescrever as aplicações (3.40) e (3.47) na forma

$$
\begin{array}{ccc}
P \times_{\Gamma} \mathfrak{g} & \longrightarrow P \times_{\Gamma} \mathfrak{X}(Q) \\
{[p, X]} & \longmapsto\left[p, X_{Q}\right]
\end{array}
$$

e

$$
\begin{aligned}
\pi^{*}\left(P \times_{\Gamma} \mathfrak{g}\right) & \longrightarrow P \times_{\Gamma} T Q \\
([p, q],[p, X]) & \longmapsto
\end{aligned}
$$

em que sua derivação das aplicações (3.39) e (3.46) se torna manifesta.

Observação 3.5 Uma situação típica em que podemos observar a coexistência de uma ação de um grupo de Lie e de uma ação de um fibrado de grupos de Lie sobre o mesmo objeto ocorre no próprio fibrado principal $P$. Por um lado, $P$ vem munido de uma ação à direita do seu grupo estrutural $G$, no sentido usual:

$$
\begin{gathered}
P \times G \quad \longrightarrow P \\
(p, g) \quad \longmapsto p \cdot g
\end{gathered}
$$


Por outro lado, $P$ também vem munido de uma ação à esquerda do fibrado de grupos de Lie $P \times{ }_{G} G$, definido conforme a equação (3.38) com $G$ agindo sobre $Q=G$ por multiplicação à esquerda: tendo em vista que o fibrado associado a $P$ por esta ação de $G$ é o próprio $P$, ela é dada por

$$
\begin{array}{ccc}
\left(P \times_{G} G\right) \times_{M} P & \longrightarrow & P \\
\left([p, g], p \cdot g_{0}\right) & \longmapsto & p \cdot\left(g g_{0}\right)
\end{array}
$$

Nota-se que estas duas ações comutam. Assim, conseguimos realizar a idéia de "localizar" a ação do grupo $\Gamma\left(P \times_{G} G\right)$ das transformações de calibre (veja a eq. (3.16)), originalmente definido como o grupo dos automorfismos estritos de $P$, nos pontos do espaço-tempo, como resultado de uma ação do fibrado de grupos de Lie $P \times_{G} G$ sobre o próprio fibrado principal $P$ e sobre todos os seus fibrados associados, o que não seria possível utilizando apenas o conceito tradicional de ações de grupos de Lie.

\subsection{Ações induzidas}

Como no caso de ações de grupos de Lie em variedades, uma ação de um fibrado de grupos de Lie em um fibrado induz ações deste mesmo em vários fibrados derivados, tais como o fibrado vertical ou o fibrado dos jatos linearizado:

Proposição 3.1 Uma ação de um fibrado de grupos de Lie $\bar{G}$ em um fibrado E, ambos sobre a mesma variedade base $M$, induz de maneira natural uma ação de $\bar{G}$ no fibrado vertical $V E$ de $E$ e outra no fibrado dos jatos linearizado $\vec{J} E$ de $E$.

Explicitamente, a primeira destas ações induzidas é definida por

$$
\left.g \cdot \frac{d}{d t} e(t)\right|_{t=0}=\left.\frac{d}{d t}(g \cdot e(t))\right|_{t=0} \quad \text { para } m \in M, g \in \bar{G}_{m}, e \text { curva em } E_{m} .
$$

enquanto que a segunda é obtida tomando o produto tensorial desta com a ação trivial de $\bar{G}$ no fibrado cotangente $T^{*} M$ de $M$, usando que $\overrightarrow{J E} \cong \pi^{*}\left(T^{*} M\right) \otimes V E$ (veja a equação (2.82)).

Como propriedade fundamental de fibrados de grupo de Lie que permite utilizá-los para formalizar os conceitos de simetria global e local em teorias de calibre, notamos os seguintes fatos:

Teorema 3.1 Dado um fibrado de grupos de Lie $\bar{G}$ sobre $M$, o fibrado dos jatos J $\bar{G}$ de primeira ordem de $\bar{G}$ é novamente um fibrado de grupos de Lie sobre $M .^{3}$

\footnotetext{
${ }^{3}$ Note que uma afirmação análoga para fibrados principais seria incorreta: $P$ sendo um fibrado principal não implica que $J P$ seria um fibrado principal.
} 
Teorema 3.2 Uma ação de um fibrado de grupos de Lie $\bar{G}$ em um fibrado E, ambos sobre a mesma variedade base $M$, induz de maneira natural uma ação de $J \bar{G}$ em $J E$.

Estes teoremas generalizam, para a teoria dos fibrados e dos fibrados de grupos de Lie, proposições bem conhecidas da teoria das variedades e dos grupos de Lie, a saber, que o fibrado tangente $T G$ de um grupo de Lie $G$ é novamente um grupo de Lie e que a partir de uma ação de um grupo de Lie $G$ sobre uma variedade $Q$ obtemos uma ação induzida do fibrado tangente $T G$ de $G$ sobre o fibrado tangente $T Q$ de $Q$ (às quais se reduzem quando a variedade base $M$ for a reta real $\mathbb{R}$ e todos os fibrados envolvidos forem explicitamente trivializados). Os argumentos usados na demonstração também são completamente análogos. De fato, recordamos que o produto e a inversão de $T G$ e a ação de $T G$ em $T Q$ são obtidos aplicando o funtor tangente ao produto e à inversão de $G$ e à ação de $G$ em $Q$, respectivamente, usando o isomorfismo canônico $T(X \times Y) \cong T X \times T Y$. Do mesmo modo, usando o isomorfismo (2.87), aplicamos o funtor $J$ ao produto (3.18) e à inversão (3.19) de um fibrado de grupos de Lie $\bar{G}$ sobre uma variedade base $M$ para obter um produto

$$
J \bar{G} \times_{M} J \bar{G} \longrightarrow J \bar{G}
$$

em $J \bar{G}$, para o qual o jato da seção unidade em $\bar{G}$ configura uma seção unidade, e uma inversão

$$
J \bar{G} \longrightarrow J \bar{G}
$$

em $J \bar{G}$, e aplicamos o funtor $J$ à ação (3.30) de um fibrado de grupos de Lie $\bar{G}$ em um fibrado $E$, ambos sobre a mesma variedade base $M$, para obter uma ação

$$
J \bar{G} \times_{M} J E \longrightarrow J E
$$

de $J \bar{G}$ em $J E$. Explicitamente, dado um ponto $m$ de $M$ e dadas seções $\phi_{1}, \phi_{2}$ e $\phi$ de $\bar{G}$ e uma seção $\varphi$ de $E$, todas definidas sobre uma vizinhança aberta de $m$, as aplicações (3.53), (3.54) e (3.55) são dadas por

$$
j_{m}\left(\phi_{1}\right) j_{m}\left(\phi_{2}\right)=j_{m}\left(\phi_{1} \phi_{2}\right),
$$

$\mathrm{e}$

$$
j_{m}(\phi)^{-1}=j_{m}\left(\phi^{-1}\right),
$$

$\mathrm{e}$

$$
j_{m}(\phi) \cdot j_{m}(\varphi)=j_{m}(\phi \cdot \varphi),
$$

respectivamente: observe que estas prescrições estão bem definidas devido à regra da cadeia. Para completar a demonstração, resta verificar a condição de que estas estruturas induzidas são localmente constantes, o que pode ser feito de modo completamente geral usando o fato de que foram obtidas a partir de estruturas localmente constantes. Contudo, tendo em vista que 
a nossa meta principal neste trabalho é utilizar o conceito de fibrados de grupo de Lie para "localizar", nos pontos do espaço-tempo, a ação do grupo das transformações de calibre, ou seja, do grupo $\operatorname{Gau}(P)$ dos automorfismos estritos do fibrado principal $P$ subjacente, estaremos interessados principalmente na situação em que os fibrados $\bar{G}$ e $E$ são associados a um mesmo fibrado principal $P$ com grupo estrutural $G$, de modo que $\bar{G}=P \times_{G} G$ e $E=P \times{ }_{G} Q$. Neste caso, dadas uma seção $\sigma$ de $P$ sobre $U$, podemos usar as trivializações induzidas de $P \times_{G} G$ e de $P \times_{G} Q$ sobre $U$ para representar secões $g_{1}, g_{2}$ e $g$ de $P \times_{G} G$ sobre $U$ por funções $\left(g_{1}\right)_{\sigma} \in C^{\infty}(U, G),\left(g_{2}\right)_{\sigma} \in C^{\infty}(U, G)$ e $g_{\sigma} \in C^{\infty}(U, G)$, respectivamente, e seções $\varphi$ de $P \times_{G} Q$ sobre $U$ por funções $\varphi_{\sigma} \in C^{\infty}(U, Q)$, conforme

$$
g_{1}=\left[\sigma,\left(g_{1}\right)_{\sigma}\right], \quad g_{2}=\left[\sigma,\left(g_{2}\right)_{\sigma}\right], \quad g=\left[\sigma, g_{\sigma}\right], \quad \varphi=\left[\sigma, \varphi_{\sigma}\right],
$$

de modo que

$$
g_{1} g_{2}=\left[\sigma,\left(g_{1}\right)_{\sigma}\left(g_{2}\right)_{\sigma}\right], \quad g^{-1}=\left[\sigma, g_{\sigma}^{-1}\right], \quad g \cdot \varphi=\left[\sigma, g_{\sigma} \cdot \varphi_{\sigma}\right] .
$$

Assim, podemos usar uma ligeira modificação das trivializações induzidas de $J\left(P \times_{G} G\right)$ e de $J\left(P \times_{G} Q\right)$ sobre $U$, conforme definidas nas equações (2.57) (para $k=1$, combinada com o isomorfismo (2.67)) e (2.54), no sentido de reverter o uso da carta $x$, para representar, em cada ponto $m$ de $U, j_{m} g_{1}, j_{m} g_{2}, j_{m} g$ e $j_{m} \varphi$ por $\left(m, g_{1}, \alpha_{1}\right),\left(m, g_{2}, \alpha_{2}\right),(m, g, \alpha)$ e $\left(m, q, T_{m} \varphi_{\sigma}\right)$, respectivamente, onde $g_{1}=\left(g_{1}\right)_{\sigma}(m), g_{2}=\left(g_{2}\right)_{\sigma}(m), g=g_{\sigma}(m) \in G$, $q=\varphi_{\sigma}(m) \in Q$ e $\alpha_{1}=T_{m}\left(g_{1}\right)_{\sigma} g_{1}^{-1}, \alpha_{2}=T_{m}\left(g_{2}\right)_{\sigma} g_{2}^{-1}, \alpha=T_{m} g_{\sigma} g^{-1} \in L\left(T_{m} M, \mathfrak{g}\right)$; então $j_{m} g_{1} j_{m} g_{2}=j_{m}\left(g_{1} g_{2}\right),\left(j_{m} g\right)^{-1}=j_{m}\left(g^{-1}\right)$ e $j_{m} g \cdot j_{m} \varphi=j_{m}(g \cdot \varphi)$ serão representados por $\left(m, g_{1} g_{2}, T_{m}\left(\left(g_{1}\right)_{\sigma}\left(g_{2}\right)_{\sigma}\right)\left(g_{1} g_{2}\right)^{-1}\right),\left(m, g^{-1}, T_{m} g_{\sigma}^{-1} g\right)$ e $\left(m, g \cdot q, T_{m}\left(g_{\sigma} \cdot \varphi_{\sigma}\right)\right)$, respectivamente, com

$$
T_{m}\left(\left(g_{1}\right)_{\sigma}\left(g_{2}\right)_{\sigma}\right)\left(g_{1} g_{2}\right)^{-1}=\alpha_{1}+\operatorname{Ad}\left(g_{1}\right) \alpha_{2},
$$

(veja a equação $(2.70)$ ) e

$$
T_{m} g_{\sigma}^{-1} g=-\operatorname{Ad}\left(g^{-1}\right) \alpha
$$

e

$$
T_{m}\left(g_{\sigma} \cdot \varphi_{\sigma}\right)=g \cdot T_{m} \varphi_{\sigma}+\alpha_{Q}(g \cdot q)
$$

(veja a equação (2.76)).

Voltando, mais uma vez, à estratégia geral de usar fibrados de grupos de Lie para "localizar", nos pontos do espaço-tempo, a ação do grupo das transformações de calibre, ou seja, do grupo $\operatorname{Gau}(P)$ dos automorfismos estritos do fibrado principal $P$ subjacente, observamos que a maior dificuldade técnica desta estratégia reside no fato de que a sua implementação pode requerer a passagem para fibrados de jatos de ordem maior do que a original. A título de exemplo, considere a ação do grupo $\Gamma\left(P \times_{G} G\right)$ das transformações de calibre (veja a eq. (3.16)) no espaço $\Gamma(C P)$ das conexões principais (veja a Proposição 2.2). Inicialmente, poderíamos imaginar que esta seria simplesmente o resultado de uma ação do fibrado de grupos de Lie $P \times_{G} G$ no fibrado $C P$. No entanto, isso não é o caso, como pode ser evidenciado a partir da seguinte proposição, que é bem conhecida (ver [5]): 
Proposição 3.2 Seja $P$ um fibrado principal sobre $M$ com grupo estrutural $G$. Representando uma transformação de calibre por um automorfismo (estrito) $f$ de $P$ ou, de forma equivalente, por uma função equivariante $\tilde{f}$ sobre $P$ a valores em $G$ e uma conexáo principal sobre $P$ por sua forma de conexão $A$, a transformada de $A$ por $f$ ou $\tilde{f}$ é dada por

$$
\left(f^{-1 *} A\right)_{p}=\operatorname{Ad}_{\tilde{f}(p)} A-d \tilde{f}(p) \tilde{f}(p)^{-1}
$$

Obviamente, o que é necessário para implementar esta lei de transformação pontualmente é incluir não apenas os valores da transformação de calibre em cada ponto do espaço-tempo, mas também os valores de suas primeiras derivadas. Isso nos leva a considerar o fibrado $J\left(P \times_{G} G\right)$ dos jatos de primeira ordem de $P \times_{G} G$. Felizmente, como vimos acima, este também é um fibrado de grupos de Lie.

Finalmente, notamos a seguinte generalização dos Teoremas 3.1 e 3.2 :

Teorema 3.3 Dado um fibrado de grupos de Lie $\bar{G}$ sobre $M$, o fibrado dos jatos $J^{k} \bar{G}$ de $k$-ésima ordem de $\bar{G}$ é novamente um fibrado de grupos de Lie sobre $M$.

Teorema 3.4 Uma ação de um fibrado de grupos de Lie $\bar{G}$ em um fibrado E, ambos sobre a mesma variedade base $M$, induz de maneira natural uma ação de $J^{k} \bar{G}$ em $J^{k} E$.

A demonstração funciona da mesma forma que no caso $k=1$, usando o fato (que não provamos aqui) que $J^{k}$ também é um funtor. Assim, temos

$$
j_{m}^{k}\left(\phi_{1}\right) j_{m}^{k}\left(\phi_{2}\right)=j_{m}^{k}\left(\phi_{1} \phi_{2}\right),
$$

e

$$
j_{m}^{k}(\phi)^{-1}=j_{m}^{k}\left(\phi^{-1}\right),
$$

$\mathrm{e}$

$$
j_{m}^{k}(\phi) \cdot j_{m}^{k}(\varphi)=j_{m}^{k}(\phi \cdot \varphi),
$$

respectivamente: observe, mais uma vez, que estas prescrições estão bem definidas devido à regra da cadeia, segundo a qual o $k$-jato de $\phi_{1} \phi_{2}$ e de $\phi \cdot \varphi$ em $m$ depende, respectivamente, apenas dos $k$-jatos de $\phi_{1}$ e $\phi_{2}$ e dos $k$-jatos de $\phi$ e $\varphi$ em $m$. (Para uma formulação explícita da regra da cadeia para derivadas de ordem superior, em termos de representações locais, veja $[2$, Teorema 1.4, p. 3].)

Utilizaremos as técnicas desenvolvidas acima para definir a ação do fibrado de grupos de Lie $J\left(P \times_{G} G\right)$ no fibrado das conexões $C P$ e a ação derivada do fibrado de grupos de Lie $J^{2}\left(P \times{ }_{G} G\right)$ no fibrado dos jatos de conexões $J(C P)$ que formalizam as leis de transformação de conexões e de suas derivadas sob transformações de calibre (veja a Proposição 3.2 acima). 


\subsubsection{Fibrado das conexões}

Para definir a ação do fibrado de grupos de Lie $J\left(P \times_{G} G\right)$ no fibrado das conexões $C P$ mencionada acima, recordamos a Observação 3.5, onde introduzimos a ação (3.51) do fibrado de grupos de Lie $P \times_{G} G$ em $P$, que comuta com a ação (3.50) de $G$ em $P$, e usamos a mesma notação para descrever as ações induzidas em nível de seções locais,

$$
\begin{aligned}
\Gamma\left(P \times\left._{G} G\right|_{U}\right) \times \Gamma\left(\left.P\right|_{U}\right) & \longrightarrow \Gamma\left(\left.P\right|_{U}\right) \\
(g, \sigma) & \longmapsto g \cdot \sigma
\end{aligned}
$$

e

$$
\begin{aligned}
& \Gamma\left(\left.P\right|_{U}\right) \times G \longrightarrow \Gamma\left(\left.P\right|_{U}\right) \\
& (\sigma, g) \longmapsto \sigma \cdot g
\end{aligned}
$$

Conforme o Teorema 3.2, aplicando o funtor $J$ à primeira, obtemos uma ação

$$
\begin{array}{ccc}
J\left(P \times_{G} G\right) \times_{M} J P & \longrightarrow & J P \\
\left(j_{m} g, j_{m} \sigma\right) & \longmapsto & j_{m} g \cdot j_{m} \sigma=j_{m}(g \cdot \sigma)
\end{array}
$$

do fibrado de grupos de Lie $J\left(P \times_{G} G\right)$ em $J P$. Por outro lado, a segunda induz de maneira natural uma ação

$$
\begin{aligned}
& J P \times G \longrightarrow J P \\
& j_{m} \sigma \cdot g \longmapsto j_{m}(\sigma \cdot g)
\end{aligned}
$$

de $G$ em $J P$ cujo quociente, como foi visto na Seção 2.3.3, define o fibrado $C P$. Note que estas duas ações comutam, pois o funtor $J$ preserva a comutatividade das ações (3.51) e (3.50) das quais elas provêm, o que permite passar a uma ação

$$
\begin{array}{ccc}
J\left(P \times_{G} G\right) \times_{M} C P & \longrightarrow & C P \\
\left(j_{m} g,\left[j_{m} \sigma\right]\right) & \longmapsto & j_{m} g \cdot\left[j_{m} \sigma\right]=\left[j_{m}(g \cdot \sigma)\right]
\end{array}
$$

do fibrado de grupos de Lie $J\left(P \times_{G} G\right)$ no fibrado quociente $C P=J P / G$, sendo que escreveremos a ação induzida em nível de seções locais na seguinte forma:

$$
\begin{array}{cl}
\Gamma\left(J\left(P \times_{G} G\right) \mid U\right) \times \Gamma\left(\left.C P\right|_{U}\right) & \longrightarrow \Gamma\left(\left.C P\right|_{U}\right) \\
((g, \partial g), A) & \longmapsto(g, \partial g) \cdot A
\end{array}
$$

Localmente, em termos de trivializações dos fibrados envolvidos provindas de uma seção local fixa $\sigma$ de $P$ sobre um aberto $U$ de $M$, podemos expressar estas ações da seguinte maneira: representando a seção $g$ de $P \times_{G} G$ sobre $U$ pela função $g_{\sigma} \in C^{\infty}(U, G)$, conforme $g=\left[\sigma, g_{\sigma}\right]$, e a seção $A$ ou $\Gamma^{A}$ de $C P$ sobre $U$ pela 1-forma $A^{\sigma} \in \Omega^{1}(U, \mathfrak{g})$ e, alternativamente, através de uma função $g^{A, \sigma} \in C^{\infty}(U \times U, G)$, conforme explicado no final da Seção 2.3.3, 
vemos que, em cada ponto $m$ de $U, j_{m} g$ corresponde a $(m, g, \alpha)$, onde $g=g_{\sigma}(m)$ e $\alpha=T_{m} g_{\sigma} g^{-1} \in L\left(T_{m} M, \mathfrak{g}\right),\left[j_{m}\left(\sigma \cdot g^{A, \sigma}(., m)\right)\right]$ corresponde a $A^{\sigma}(m)=-T_{m} g^{A, \sigma}(., m)$ $\in L\left(T_{m} M, \mathfrak{g}\right)$ e portanto

$j_{m} g \cdot\left[j_{m}\left(\sigma \cdot g^{A, \sigma}(., m)\right)\right]=j_{m}\left[\sigma, g_{\sigma}\right] \cdot\left[j_{m}\left(\sigma \cdot g^{A, \sigma}(., m)\right)\right]=\left[j_{m}\left(\sigma \cdot\left(g_{\sigma} g^{A, \sigma}(., m) g^{-1}\right)\right)\right]$

corresponde a $\left(g_{\sigma}(m), \partial g_{\sigma}(m)\right) \cdot A^{\sigma}(m)=-T_{m}\left(g_{\sigma} g^{A, \sigma}(., m) g^{-1}\right) \in L\left(T_{m} M, \mathfrak{g}\right)$, o que após aplicação da regra do produto (e remoção do ponto $m$, que é arbitrário) recupera a lei de transformação clássica

$$
\left(g_{\sigma}, \partial g_{\sigma}\right) \cdot A^{\sigma}=g_{\sigma} A^{\sigma} g_{\sigma}^{-1}-\partial g_{\sigma} g_{\sigma}^{-1}
$$

que é a expressão local da ação (3.72).

\subsubsection{Fibrado dos jatos de conexões}

A extensão natural desta ação aos fibrados de jatos de $C P$ nos levaria a uma ação de $J\left(J\left(P \times_{G} G\right)\right)$ em $J(C P)$. Porém, como o que nos interessa é o resultado de derivadas da ação de Aut(P) em $C P$ (ou seja, queremos 2-jatos de $P \times{ }_{G} G$ e não seções de $J\left(J\left(P \times_{G} G\right)\right)$ ), desejaríamos considerar uma ação de $J^{2}\left(P \times_{G} G\right)$ em $J(C P)$ obtida como uma restrição dessa ação de $J\left(J\left(P \times_{G} G\right)\right)$ em $J(C P)$.

De fato, esta ação se restringe a uma ação de $J^{2}\left(P \times_{G} G\right)$ em $J(C P)$, já que $J^{2}\left(P \times_{G} G\right)$ pode ser considerado um subfibrado de grupos de Lie de $J\left(J\left(P \times_{G} G\right)\right)$ pela inclusão

$$
\begin{array}{clc}
J^{2}\left(P \times_{G} G\right) & \longrightarrow J\left(J\left(P \times_{G} G\right)\right) \\
j_{m}^{2} \phi & \longmapsto & j_{m}^{1}\left(j^{1} \phi\right)
\end{array} .
$$

A restrição da ação de $J\left(J\left(P \times_{G} G\right)\right)$ a $J^{2}\left(P \times_{G} G\right)$ é então dada por

$$
j_{m}^{2} \phi \cdot j_{m}^{1} A=j_{m}^{1}\left(j^{1} \phi \cdot A\right) .
$$

Assim, a invariância por transformações de calibre de uma lagrangiana em $J(C P)$ será expressa em termos da invariância sob a ação do fibrado $J^{2}\left(P \times_{G} G\right)$. Para examinarmos como esta ação se expressa localmente, consideramos novamente uma seção $\sigma \in \Gamma\left(\left.P\right|_{U}\right)$ em conjunto com uma carta $x: U \rightarrow U_{0}$ de $M$, onde $U_{0}$ é uma vizinhança aberta de $0 \mathrm{em} \mathbb{R}^{n}$. Primeiramente, recordamos (ver seção 2.2 .2 (página 42)) que esses objetos induzem uma trivialização

$$
\begin{aligned}
J^{2} \Phi_{\sigma}^{x}:\left.\quad J^{2}\left(P \times_{G} G\right)\right|_{U} & \longrightarrow U \times J_{0}^{2}\left(\mathbb{R}^{n}, G\right) \\
j_{m}^{2} g & \longmapsto\left(m, j_{0}^{2}\left(g_{\sigma} \circ x^{-1}\right)\right)
\end{aligned}
$$


de $J^{2}\left(P \times_{G} G\right)$ sobre $U$, onde novamente a aplicação $g_{\sigma} \in C^{\infty}(U, G)$ representa a seção $g \in \Gamma\left(P \times\left._{G} G\right|_{U}\right)$ no sentido de que vale a equação (2.58).

Em segundo lugar, aplicando (2.51) sobre (2.54), ambas para o caso $k=1$ e $Q=G$, obtemos que $\sigma$ e $x$ induzem a trivialização

$$
\begin{array}{clc}
J\left(J \Phi_{\sigma}^{x}\right)^{x}:\left.J\left(J\left(P \times_{G} G\right)\right)\right|_{U} & \longrightarrow & U \times J_{0}^{1}\left(\mathbb{R}^{n}, G \ltimes L\left(\mathbb{R}^{n}, \mathfrak{g}\right)\right) \\
j_{m} g^{(1)} & \longmapsto\left(m, j_{0}^{1}\left(\operatorname{pr}_{2} \circ J \Phi_{\sigma}^{x} \circ g^{(1)} \circ x^{-1} \circ \tau_{x^{-1}(m)}\right)\right)
\end{array}
$$

de $J\left(J\left(P \times{ }_{G} G\right)\right)$ sobre $U$. Aqui, lançamos mão implicitamente do isomorfismo que identifica $J_{0}^{2}\left(\mathbb{R}^{n}, G\right) \operatorname{com} G \ltimes L\left(\mathbb{R}^{n}, \mathfrak{g}\right.$.

Combinando esta última trivialização com uma trivialização compatível de $J(C P)$,

$$
J(C \Phi)_{\sigma}^{x}:\left.\quad J(C P)\right|_{U} \longrightarrow U \times J_{0}^{1}\left(\mathbb{R}^{n}, L\left(\mathbb{R}^{n}, \mathfrak{g}\right)\right)
$$

temos que a ação de $J\left(J\left(P \times_{G} G\right)\right)$ em $J(C P)$ claramente se traduz na ação de $j_{0}^{1} \boldsymbol{g}^{(1)} \in J_{0}^{1}\left(\mathbb{R}^{n}, G \ltimes L\left(\mathbb{R}^{n}, \mathfrak{g}\right)\right)$ em $j_{0}^{1} \boldsymbol{\alpha} \in J_{0}^{1}\left(\mathbb{R}^{n}, L\left(\mathbb{R}^{n}, \mathfrak{g}\right)\right)$ dada por

$$
j_{0}^{1} \boldsymbol{g}^{(1)} \cdot j_{0}^{1} \boldsymbol{\alpha}=j_{0}^{1}\left(\boldsymbol{g}^{(1)} \cdot \boldsymbol{\alpha}\right),
$$

onde $\boldsymbol{g}^{(1)} \in C^{\infty}\left(\mathbb{R}^{n}, G \ltimes L\left(\mathbb{R}^{n}, \mathfrak{g}\right)\right)$ e $\boldsymbol{\alpha} \in C^{\infty}\left(\mathbb{R}^{n}, L\left(\mathbb{R}^{n}, \mathfrak{g}\right)\right)$. Escrevendo $\boldsymbol{g}^{(1)}$ na forma $\boldsymbol{g}^{(1)}=(\boldsymbol{g}, \boldsymbol{\alpha})$ com $\boldsymbol{g} \in C^{\infty}\left(\mathbb{R}^{n}, G\right)$ e $\boldsymbol{\xi} \in C^{\infty}\left(\mathbb{R}^{n}, L\left(\mathbb{R}^{n}, \mathfrak{g}\right)\right)$ e aplicando a equação (3.73) em conjunto com a regra da cadeia, obtemos

$$
j_{0}^{1} \boldsymbol{g}^{(1)} \cdot j_{0}^{1} \boldsymbol{\alpha}=D_{0}(\operatorname{Ad}(\boldsymbol{g}) \circ(\boldsymbol{\alpha}+\boldsymbol{\xi})) .
$$

Pondo

$$
\begin{gathered}
\boldsymbol{g}(0)=g \quad, \quad \boldsymbol{\xi}(0)=\xi, \quad \boldsymbol{\alpha}(0)=\alpha, \\
D_{0}\left(\ln \left(g^{-1} \boldsymbol{g}\right)\right)=\xi^{\prime}, \quad D_{0} \boldsymbol{\xi}=\eta \quad D_{0} \boldsymbol{\alpha}=\beta,
\end{gathered}
$$

podemos identificar $J_{0}^{1}\left(\mathbb{R}^{n}, G \ltimes L\left(\mathbb{R}^{n}, \mathfrak{g}\right)\right)$ com o grupo

$$
\left.\left(G \ltimes L\left(\mathbb{R}^{n}, \mathfrak{g}\right)\right) \ltimes L\left(\mathbb{R}^{n}, \mathfrak{g} \ltimes L\left(\mathbb{R}^{n}, \mathfrak{g}\right)\right)=\left(G \ltimes L\left(\mathbb{R}^{n}, \mathfrak{g}\right)\right) \ltimes\left(L\left(\mathbb{R}^{n}, \mathfrak{g}\right) \times L^{2}\left(\mathbb{R}^{n}, \mathfrak{g}\right)\right)\right),
$$

obtido por iteração da equação (2.68), como segue:

$$
j_{0}^{1} \boldsymbol{g}^{(1)} \leftrightarrow\left((g, \xi),\left(\xi^{\prime}, \eta\right)\right) .
$$

Assim, a equação (3.76) se traduzirá em

$$
\left((g, \xi),\left(\xi^{\prime}, \eta\right)\right) \cdot(\alpha, \beta)=\operatorname{Ad}_{g}\left(\left[\xi^{\prime}, \alpha+\xi\right]+\beta+\eta\right) .
$$


O fato de que $J^{2}\left(P \times_{G} G\right)$ é um subfibrado de grupos de Lie de $J\left(J\left(P \times_{G} G\right)\right)$ se reflete no fato de que $J_{0}^{2}\left(\mathbb{R}^{n}, G\right)$ é um subgrupo de $J_{0}^{1}\left(\mathbb{R}^{n}, G \ltimes L\left(\mathbb{R}^{n}, \mathfrak{g}\right)\right)$, sendo que a inclusão, analogamente a (3.74), é dada por

$$
\begin{array}{ccc}
J_{0}^{2}\left(\mathbb{R}^{n}, G\right) & \longrightarrow J_{0}^{1}\left(\mathbb{R}^{n}, G \ltimes L\left(\mathbb{R}^{n}, \mathfrak{g}\right)\right) \\
\left(j_{0}^{2} \boldsymbol{g}\right) & \longmapsto & \left(j_{0}^{1}\left(\boldsymbol{g}, T \boldsymbol{g g}(0)^{-1}\right)\right)
\end{array} .
$$

Conforme provamos na seção 2.2.3, o grupo de Lie $J_{0}^{2}\left(\mathbb{R}^{n}, G\right)$ é isomorfo ao grupo de Lie $G \times L\left(\mathbb{R}^{n}, \mathfrak{g}\right) \oplus L_{s}^{2}\left(\mathbb{R}^{n}, \mathfrak{g}\right)$. Claramente, sob as identificações (2.67) e (3.77), a inclusão (3.79) se traduz na inclusão

$$
G \times L\left(\mathbb{R}^{n}, \mathfrak{g}\right) \oplus L_{s}^{2}\left(\mathbb{R}^{n}, \mathfrak{g}\right) \longrightarrow\left(G \ltimes L\left(\mathbb{R}^{n}, \mathfrak{g}\right)\right) \ltimes\left(L\left(\mathbb{R}^{n}, \mathfrak{g}\right) \times L^{2}\left(\mathbb{R}^{n}, \mathfrak{g}\right)\right),
$$

dada por

$$
(g, \xi, \eta) \mapsto((g, \xi),(\xi, \eta)) .
$$

Portanto, a ação de $G \times L\left(\mathbb{R}^{n}, \mathfrak{g}\right) \oplus L_{s}^{2}\left(\mathbb{R}^{n}, \mathfrak{g}\right)$ em $L\left(\mathbb{R}^{n}, \mathfrak{g}\right) \times L^{2}\left(\mathbb{R}^{n}, \mathfrak{g}\right)$ obtida pela restrição de (3.78) é dada por

$$
\begin{gathered}
(g, \xi, \eta) \cdot(\alpha, \beta)=\left(\operatorname{Ad}_{g}(\alpha+\xi), \operatorname{Ad}_{g}([\xi, \alpha+\xi]+\beta+\eta)\right) \\
\operatorname{para}(g, \xi, \eta) \in G \times L\left(\mathbb{R}^{n}, \mathfrak{g}\right) \oplus L_{s}^{2}\left(\mathbb{R}^{n}, \mathfrak{g}\right),(\alpha, \beta) \in L\left(\mathbb{R}^{n}, \mathfrak{g}\right) \times L^{2}\left(\mathbb{R}^{n}, \mathfrak{g}\right) .
\end{gathered}
$$

\subsubsection{Fibrado dos cojatos}

Aqui, damos o primeiro passo para generalizar o conceito de simetria local da mecânica não autonoma para a teoria dos campos - ou seja, para o caso em que o espaço de fase é $J^{\circledast} E$ em vez de $\mathbb{R} \times T^{*} Q \times \mathbb{R}$ e em que temos, no lugar de uma ação de $\mathbb{R} \times(G \ltimes \mathfrak{g})$ em $\mathbb{R} \times T^{*} Q \times \mathbb{R}$ induzida por uma ação de $G$ em $Q$, uma ação de $J \bar{G}$ em $J^{\circledast} E$ induzida por uma ação de $\bar{G}$ em $E$.

Analogamente à situação que ocorre na mecânica (caso em que $\bar{G}$ é o fibrado principal trivial $\mathbb{R} \times G$ ), a ação de $J \bar{G}$ em $J^{\circledast} E$ é a aplicação dual afim da ação ... de $J \bar{G}$ em $J E$, ou seja, é a aplicação definida de maneira que

$$
\begin{gathered}
\left\langle j_{m} g \cdot z, j_{m} g \cdot j_{m} \phi\right\rangle=\left\langle z, j_{m} \phi\right\rangle \\
\text { para } j_{m} g \in J \bar{G}_{m}, z \in J^{\circledast} E_{m}, j_{m} \phi \in J E_{m},
\end{gathered}
$$

a última equação é satisfeita se e somente se, nas fibras, é satisfeita a equação

$$
\begin{gathered}
\left\langle(g, \xi) \cdot\left(q, \alpha_{q}, p\right),(g, \xi) \cdot\left(q, v_{q}\right)\right\rangle=\left\langle\left(q, \alpha_{q}, p\right),\left(q, v_{q}\right)\right\rangle \\
\text { para todo }(g, \xi) \in G \ltimes L\left(\mathbb{R}^{n}, \mathfrak{g}\right), q \in Q, v_{q} \in L\left(\mathbb{R}^{n}, T_{q} Q\right), \alpha_{q} \in L\left(\mathbb{R}^{n}, T_{q} Q\right)^{*}, p \in \mathbb{R} .
\end{gathered}
$$


Esta ação é, portanto, dada fibra a fibra pela ação de $G \ltimes L\left(\mathbb{R}^{n}, \mathfrak{g}\right)$ em $\left(T^{n} Q\right)^{*} \times \mathbb{R}$ definida por

$$
\begin{gathered}
(g, \xi) \cdot\left(q, \alpha_{q}, p\right)=\left(g \cdot q, \alpha_{q} \circ T_{q} L_{g^{-1}}^{Q}, p-\alpha_{q}\left(\xi_{q}^{Q}\right)\right) \\
\operatorname{para}(g, \xi) \in G \ltimes L\left(\mathbb{R}^{n}, \mathfrak{g}\right), q \in Q, \alpha_{q} \in L\left(\mathbb{R}^{n}, T_{q} Q\right)^{*}, p \in \mathbb{R} .
\end{gathered}
$$

Se, além das coordenadas locais $x^{\mu}$ para $M$ e das trivializações locais de $\bar{G}$ e de $E$, considerarmos coordenadas locais $g^{i}$ para $G$ e $q^{i}$ para $Q$, então em termos das coordenadas locais induzidas $\left(x^{\mu}, g^{i}\right)$ de $\bar{G},\left(x^{\mu}, q^{i}\right)$ de $E,\left(x^{\mu}, g^{i}, g_{\mu}^{i}\right)$ de $J \bar{G}$ e $\left(x^{\mu}, q^{i}, p_{\mu}^{i}, p\right)$ de $J^{\circledast} E$, a ação acima assume a expressão local

\subsection{Grupóides de Lie, ações}

O conceito de ação de um fibrado de grupos de Lie permite "localizar" a ação de transformações de calibre representadas por automorfismos estritos de um fibrado principal. Se quisermos extender os resultados da seção anterior para todos os automorfismos, unificando simetrias locais espaço-temporais e simetrias locais internas, é necessário lançar mão de uma generalização desse conceito, a saber, as noções de grupóides de Lie e de ações de grupóides de Lie em fibrados.

\subsubsection{Grupóides de Lie}

Definição 3.5 Um grupóide de Lie $\mathcal{G}$ sobre uma variedade $M$ é uma variedade $\mathcal{G}$ munida de:

- duas submersões sobrejetoras $s, t: \mathcal{G} \rightarrow M$, a projeção fonte ("source") $s$ e a projeção alvo ("target") $t$. Pensamos os elementos $g$ de $\mathcal{G}$ como flechas $t(g) \stackrel{g}{\longleftarrow} s(g)$.

- um produto associativo dado por uma função diferenciável

$$
\begin{gathered}
\mathcal{G}_{c} \longrightarrow \mathcal{G} \\
(g, h) \longmapsto g h
\end{gathered},
$$

onde

$$
\mathcal{G}_{c}=\{(g, h) \in \mathcal{G} \times \mathcal{G} \mid s(g)=t(h)\},
$$

tal que

$$
s(g h)=s(h), \quad t(g h)=t(g)
$$

e

$$
f(g h)=(f g) h \quad \text { quando } s(f)=t(g) \text { e } s(g)=t(h)
$$


- um elemento neutro dado por uma função diferenciável $1: M \rightarrow \mathcal{G}$ tal que para todo $m \in M$ e todo $g \in \mathcal{G}$, vale $s(1(m))=m=t(1(m))$ e $g 1(m)=g$ quando $s(g)=m$, $1(m) g=g$ quando $t(g)=m$;

- elementos inversos: existe uma função diferenciável $\iota: \mathcal{G} \rightarrow \mathcal{G}$ tal que para todo $g \in \mathcal{G}$, vale $s(\iota(g))=t(g), t(\iota(g))=s(g)$ e $\iota(g) g=1(s(g)), g \iota(g)=1(t(g))$.

Como caso especial, temos o conceito de um fibrado de grupos de Lie como um grupóide de Lie no qual as projeções fonte e alvo coincidem entre elas e com a projeção do fibrado.

\subsubsection{Ações de grupóides de Lie}

Definição 3.6 Seja $\mathcal{G}$ um grupóide de Lie sobre $M$ e $E$ uma variedade munida de uma aplicação $\zeta: E \rightarrow M$. Uma ação de $\mathcal{G}$ em $E$ é uma aplicação

$$
\begin{aligned}
& \mathcal{G} \times{ }_{\zeta} E=\{(g, e) \mid s(g)=\zeta(e)\} \quad \longrightarrow \quad E \\
& (g, e) \quad \longmapsto g \cdot e^{\prime}
\end{aligned}
$$

tal que $\zeta(g \cdot e)=t(g)$ e que satisfaz as identidades de uma ação:

- $g \cdot(h \cdot e)=g h \cdot e$ para todo $g, h \in \mathcal{G}, e \in E$ para os quais estas expressões fazem sentido.

- $1(\zeta(e)) \cdot e=e$, para todo $e \in E$.

Esta definição nos permite, em particular, definir a ação de um grupóide sobre $M$ em fibrados sobre $M$.

\subsubsection{Bisseções}

Uma bisseção de um grupóide de Lie $\mathcal{G}$ sobre $M$ é uma função diferenciável $\beta: M \rightarrow \mathcal{G}$ tal que $s_{\beta}=s \circ \beta$ e $t_{\beta}=t \circ \beta$ são difeomorfismos de $M$. O conjunto das bisseções de $\mathcal{G}$, denotado por $\Gamma(\mathcal{G})$, é um grupo com produto dado por

$$
\beta_{2} \beta_{1}(m)=\beta_{2}\left(t_{\beta_{1}}(m)\right) \beta_{1}\left(C_{t_{\beta_{1}}^{-1}}\left(s_{\beta_{2}}\right)(m)\right),
$$

onde, dados $f, g \in \operatorname{Diff}(M), C_{f}(g)=f \circ g \circ f^{-1}$. 


\subsubsection{Grupóide de calibre}

Dado um fibrado principal $P$ sobre uma variedade $M$ com grupo estrutural $G$, o grupóide de calibre de $P$ é o quociente de $P \times P$ pela ação (diagonal) de $G$. Sendo $\pi$ a projeção de $P$ em $M$, então as projeções deste grupóide em $M$ são dadas por

$$
s\left(\left[p_{1}, p_{2}\right]\right)=\pi\left(p_{2}\right) \quad t\left(\left[p_{1}, p_{2}\right]\right)=\pi\left(p_{1}\right)
$$

e o produto é dado por

$$
\left[p_{1}, p_{2}\right]\left[p_{2}, p_{3}\right]=\left[p_{1}, p_{3}\right] .
$$

A razão para que este grupóide seja chamado de grupóide de calibre é a existência do seguinte isomorfismo:

$$
\Gamma((P \times P) / G) \cong \operatorname{Aut}(P) \ltimes \operatorname{Diff}(M) .
$$

Em particular, considerando o subgrupo $\Gamma(\mathcal{G})_{s}$ de $\Gamma(\mathcal{G})$ formado pelas bisseções $\beta \in \mathcal{G}$ que fixam a fonte (ou seja, tais que $s \circ \beta=\operatorname{Id}_{M}$ ), temos

$$
\Gamma((P \times P) / G)_{s} \cong \operatorname{Aut}(P) .
$$

\subsection{Invariância local e global}

$\mathrm{Na}$ formulação geométrica tradicional de teorias de calibre, os potenciais de calibre são representados por seções do fibrado $C P$ das conexões principais de um fibrado principal $P$ e os campos de matéria por seções de um fibrado $P \times_{G} Q$ associado a $P$. O fibrado de configuração é portanto dado pelo produto em fibras $E=C P \times_{M}\left(P \times_{G} Q\right)$. O grupo $\operatorname{Aut}(P)$ dos automorfismos de $P$ e o subgrupo $\operatorname{Gau}(P)$ dos automorfismos estritos de $P$ agem naturalmente em $\Gamma(C P)$ e em $\Gamma\left(P \times_{G} Q\right)$ e, por conseguinte, também em $\Gamma(E)$.

Uma teoria de calibre é descrita por uma lagrangiana $\mathcal{L}: J E \rightarrow \bigwedge^{n} T^{*} M$ que, normalmente, é a soma de dois termos: uma lagrangiana $\mathcal{L}_{\text {gauge }}: J(C P) \rightarrow \Lambda^{n} T^{*} M$ só para os campos de calibre e uma lagrangiana $\mathcal{L}_{\text {mat }}: C P \times_{M} J\left(P \times_{G} Q\right) \rightarrow \bigwedge^{n} T^{*} M$ para os campos de matéria onde os potenciais de calibre aparecem apenas como campos auxiliares (i.e., sem derivadas),

$$
\mathcal{L}=\mathcal{L}_{\text {gauge }}+\mathcal{L}_{\text {mat }} .
$$

A hipótese básica em teorias de calibre é a de invariância local, ou invariância de calibre, o que significa que para todo compacto $K$ do espaço-tempo $M$, o funcional ação $\mathcal{S}_{K}: \Gamma(E) \rightarrow \mathbb{R}$ definido por integração da lagrangiana $\mathcal{L}$ sobre $K$, i.e.,

$$
\mathcal{S}_{K}[A, \varphi]=\int_{K} \mathcal{L}(j A, j \varphi)
$$


deve ser invariante sob a ação do grupo $\operatorname{Gau}(P)$, sendo que a mesma hipótese é imposta sobre as duas partes $\left(\mathcal{S}_{\text {gauge }}\right)_{K}$ e $\left(\mathcal{S}_{\text {mat }}\right)_{K}$ do funcional ação, definidas por

$$
\left(\mathcal{S}_{\text {gauge }}\right)_{K}[A]=\int_{K}\left(\mathcal{L}_{\text {gauge }}\right)(j A),
$$

e

$$
\left(\mathcal{S}_{\text {mat }}\right)_{K}[A, \varphi]=\int_{K}\left(\mathcal{L}_{\text {mat }}\right)(A, j \varphi) .
$$

Uma hipótese mais forte, que poderia ser chamada de invariância da teoria sob transformações de calibre e difeomorfismos do espaço-tempo, seria que estes funcionais sejam

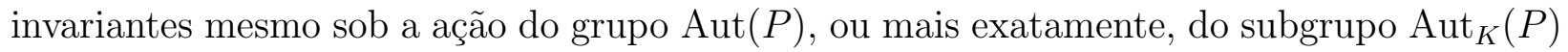
dos automorfismos de $P$ que recobrem difeomorfismos de $M$ preservando $K$ : esta condição caracteriza "teorias de calibre topológicas", o exemplo padrão sendo as teorias de ChernSimons em 3 dimensões do espaço-tempo.

Por outro lado, observamos que, como foi explicado anteriormente, existem

- uma ação natural do fibrado de grupos de Lie $J\left(P \times_{G} G\right)$ no fibrado das conexões $C P$, que induz uma ação natural do fibrado de grupos de Lie $J^{2}\left(P \times{ }_{G} G\right)$ no fibrado $J(C P)$,

- uma ação natural do fibrado de grupos de Lie $P \times{ }_{G} G$ no fibrado associado $P \times{ }_{G} Q$, que induz uma ação natural do fibrado de grupos de Lie $J\left(P \times_{G} G\right)$ no fibrado $J\left(P \times_{G} Q\right)$.

Isso posto, podemos formular os primeiros teoremas centrais desta tese.

Teorema 3.5 O funcional ação $\mathcal{S}_{\text {gauge }}$ definido por integração da lagrangiana $\mathcal{L}_{\text {gauge }}$ sobre compactos $K$ do espaço-tempo $M$ é invariante sob transformaçôes de calibre se e somente se a lagrangiana $\mathcal{L}_{\text {gauge }}$ é invariante sob a ação do fibrado de grupos de Lie $J^{2}\left(P \times_{G} G\right)$ em $J(C P)$.

Teorema 3.6 O funcional ação $\mathcal{S}_{\text {mat }}$ definido por integração da lagrangiana $\mathcal{L}_{\text {mat }}$ sobre compactos $K$ do espaço-tempo $M$ é invariante sob transformaçôes de calibre se e somente se a lagrangiana $\mathcal{L}_{\text {mat }}$ é invariante sob a ação do fibrado de grupos de Lie $J\left(P \times_{G} G\right)$ em $J\left(P \times_{G} Q\right)$.

De forma semelhante, podemos caracterizar teorias de calibre topológicas.

Teorema 3.7 O funcional ação $\mathcal{S}_{\text {gauge }}$ definido por integração da lagrangiana $\mathcal{L}_{\text {gauge }}$ sobre compactos $K$ do espaço-tempo $M$ é invariante sob transformações de calibre e difeomorfismos do espaço-tempo se e somente se a lagrangiana $\mathcal{L}_{\text {gauge }}$ for invariante sob a ação do grupóide de calibre $J^{2}(P \times P / G)$ em $J(C P)$. 
Teorema 3.8 O funcional ação $\mathcal{S}_{\text {mat }}$ definido por integração da lagrangiana $\mathcal{L}_{\text {mat }}$ sobre compactos $K$ do espaço-tempo $M$ é invariante sob transformações de calibre e difeomorfismos do espaço-tempo se e somente se a lagrangiana $\mathcal{L}_{\text {mat }}$ for invariante sob a ação do grupóide de calibre $J(P \times P / G)$ em $J\left(P \times_{G} Q\right)$.

A demonstração destas afirmações não será dada aqui, tendo em vista que isso requer, como pré-requisito, mostrar que $J(P \times P / G)$ e $J^{2}(P \times P / G)$ são de fato grupóides de Lie e especificar as suas ações mencionadas nestes últimos dois teoremas, e tendo em vista que teorias de calibre topológicas, além de "raras", são de menor importância para a física do que teorias de calibre em geral.

Resta saber como construir lagrangianas (de primeira ordem) que apresentem invariância de calibre. Grosso modo, a história do desenvolvimento das teorias de calibre, desde os seus primórdios devidos a Weyl e a Yang e Mills, traçou dois métodos para abordar esta questão. No caso de campos de matéria, existe um procedimento padrão chamado de "acoplamento mínimo", segundo o qual se deve começar considerando uma lagrangiana com invariância apenas global para então transformá-la em uma lagrangiana com invariância local pela simples regra de substituir todas as derivadas parciais comuns por derivadas covariantes, enquanto que no caso de campos de calibre, há o "teorema de Utiyama" segundo o qual lagrangianas envolvendo apenas potenciais de calibre (conexões) e suas primeiras derivadas podem ser escritas como funções apenas dos campos de calibre (curvatura). Estes dois métodos serão discutidos no próximo capítulo, mas como pré-requisito, precisamos especificar o que, no caso de campos de matéria, se entende por invariância global. Afinal, campos de matéria são seções de fibrados associados que, em geral, não carregam nenhuma ação de algum grupo de Lie.

Para formular o conceito de invariância global, consideramos a noção de uma lagrangiana reduzida, que no caso dos campos de calibre é uma função

$$
\mathcal{L}_{\text {gauge }}^{\text {red }}: \bigwedge^{2}\left(T^{*} M\right) \otimes\left(P \times_{G} \mathfrak{g}\right) \longrightarrow \bigwedge^{n}\left(T^{*} M\right)
$$

e no caso dos campos de matéria é uma função

$$
\mathcal{L}_{\text {mat }}^{\text {red }}: \vec{J}\left(P \times_{G} Q\right) \longrightarrow \bigwedge^{n}\left(T^{*} M\right)
$$

sendo que em ambos os casos, os domínios são fibrados sobre $M$ munidos de uma ação natural do fibrado de grupos de Lie $P \times{ }_{G} G$, o que permite dizer que tal lagrangiana reduzida apresenta invariância global se é invariante sob a respectiva ação. A partir desta, constróise uma lagrangiana com invariância local inserindo a curvatura (usando a decomposição (2.150), no primeiro caso, e usando conexões para estabelecer um isomorfismo entre $J\left(P \times_{G}\right.$ $Q)$ e $\vec{J}\left(P \times_{G} Q\right)$, no segundo caso. 


\begin{tabular}{|c|c|c|}
\hline & Mecânica não-autônoma & Teoria dos campos \\
\hline variedade base & tempo $\mathbb{R}$ & espaço-tempo $M$ \\
\hline grupo de simetrias globais & $G$ & $G$ \\
\hline grupo de simetrias locais & $C^{\infty}(\mathbb{R}, G)$ & $\Gamma\left(P \times_{G} G\right)$ \\
\hline curvas / campos & elementos de $C^{\infty}(\mathbb{R}, Q)$ & elementos de $\Gamma\left(P \times_{G} Q\right)$ \\
\hline $\begin{array}{l}\text { fibrado de grupos de Lie } \\
\text { para simetrias globais }\end{array}$ & $\mathbb{R} \times G$ & $P \times_{G} G$ \\
\hline $\begin{array}{l}\text { fibrado de grupos de Lie } \\
\text { para simetrias locais }\end{array}$ & $\mathbb{R} \times T G$ & $J\left(P \times_{G} G\right)$ \\
\hline $\begin{array}{l}\text { fibrado de configurações } \\
\text { para "matéria" }\end{array}$ & $\mathbb{R} \times Q$ & $P \times_{G} Q$ \\
\hline $\begin{array}{c}\text { fibrado de velocidades } \\
\text { para "matéria" }\end{array}$ & $\mathbb{R} \times T Q$ & $J\left(P \times_{G} Q\right)$ \\
\hline $\begin{array}{l}\text { ação de simetrias globais } \\
\text { em termos de fibrados }\end{array}$ & $\begin{array}{c}\text { ação de } \mathbb{R} \times G \\
\text { em } \mathbb{R} \times Q \text { e } \mathbb{R} \times T Q\end{array}$ & $\begin{array}{c}\text { ação de } P \times_{G} G \\
\text { em } P \times_{G} Q \text { e } \vec{J}\left(P \times_{G} Q\right)\end{array}$ \\
\hline $\begin{array}{l}\text { ação de simetrias locais } \\
\text { em termos de fibrados }\end{array}$ & $\begin{array}{l}\text { ação de } \mathbb{R} \times T G \\
\text { em } \mathbb{R} \times T Q\end{array}$ & $\begin{array}{l}\text { ação de } J\left(P \times_{G} G\right) \\
\quad \text { em } J\left(P \times_{G} Q\right)\end{array}$ \\
\hline invariância global & $\begin{array}{l}\text { invariância sob ação } \\
\text { de } G \text {, ou } \mathbb{R} \times G\end{array}$ & invariância sob ação de $P \times_{G} G$ \\
\hline invariância local & $\begin{array}{c}\text { invariância sob ação } \\
\text { de } T G, \text { ou } \mathbb{R} \times T G\end{array}$ & invariância sob ação de $J\left(P \times_{G} G\right)$ \\
\hline
\end{tabular}


CAPÍTULO 4

\section{Acoplamento Mínimo e Teorema de Utiyama}

O mecanismo de acoplamento mínimo constitui um procedimento padrão para construir, a partir de uma lagrangiana dada para um conjunto de "campos de matéria" e que é globalmente invariante sob a ação de um determinado grupo de simetria, uma nova lagrangiana que é localmente invariante sob transformações de calibre associadas a este grupo. Essencialmente, o método consiste em "substituir derivadas comuns por derivadas covariantes", o que requer introduzir um "potencial de calibre", inicialmente sem dinâmica própria, ou seja, como campo auxiliar. ${ }^{1}$ Cabe observar e até enfatizar o fato de que esta prescrição é única em teorias de primeira ordem (i.e., teorias onde a lagrangiana depende apenas dos campos e de suas derivadas de primeira ordem), pois caso contrário, aparece um problema de ordenamento para derivadas covariantes (que na presença de curvatura deixam de comutar). Esta unicidade do mecanismo de acoplamento mínimo para estender simetrias globais a simetrias locais constitui um dos argumentos mais incisivos em favor do papel de destaque para teorias de primeira ordem na física. Num segundo passo, o potencial de calibre é elevado do status de um campo auxiliar para o de um campo dinâmico, o que requer introduzir uma lagrangiana, também de primeira ordem, puramente para o potencial de calibre e invariante sob transformações de calibre. Tais lagrangianas são completamente classificadas pelo teorema de Utiyama, que afirma que elas dependem apenas do correspondente campo de calibre. ${ }^{2}$

Nossa meta neste capítulo é formalizar o mecanismo de acoplamento mínimo e formular o teorema de Utiyama, assim como a unificação dos dois, que se refere a teorias contendo campos de matéria e campos de calibre dinâmicos, com rigor matemático, empregando os

\footnotetext{
${ }^{1} \mathrm{Na}$ mecânica, tais variáveis sem dinâmica própria são conhecidas como "parâmetros de Lagrange".

${ }^{2}$ Em terminologia matemática, potenciais de calibre $A_{\mu}$ representam uma conexão e campos de calibre $F_{\mu \nu}$ representam o correspondente tensor de curvatura.
} 
conceitos desenvolvidos nas seções anteriores. Como veremos, estes princípios permitem uma caracterização completa das lagrangianas de primeira ordem que são invariantes sob transformações de calibre, afirmando que estas lagrangianas dependem, respectivamente, apenas dos campos de matéria $\varphi^{i}$ e suas primeiras derivadas covariantes $D_{\mu} \varphi^{i}$ e dos campos de calibre $F_{\mu \nu}$.

Tecnicamente, a demonstração dos teoremas pertinentes baseia-se na seguinte proposição sobre fatorização de aplicações equivariantes [19, p. 233]:

Proposição 4.1 Sejam $G_{1}, G_{2}, G_{3}$ grupos de Lie e $X_{1}, X_{2}, X_{3}$ variedades tais que $G_{i}$ age à esquerda sobre $X_{i}(1 \leqslant i \leqslant 3)$. Sejam $\chi_{i j}: G_{i} \rightarrow G_{j}(1 \leqslant i<j \leqslant 3)$ homomorfismos de grupos de Lie tais que $\chi_{23}{ }^{\circ} \chi_{12}=\chi_{13}$, seja $K_{12}=\operatorname{ker} \chi_{12}$, e seja $\pi: X_{1} \rightarrow X_{2}$ uma submersão sobrejetora $\chi_{12}$-equivariante tal que $K_{12}$ age transitivamente sobre as suas fibras. Então a fórmula

$$
f_{13}=f_{23} \circ \pi
$$

estabelece uma bijeção entre as aplicações $\chi_{13}$-equivariantes $f_{13}: X_{1} \rightarrow X_{3}$ e as aplicações $\chi_{23}$ equivariantes $f_{23}: X_{2} \rightarrow X_{3}$.

Podemos expressar esta afirmação por meio dos seguintes dois diagramas comutativos:
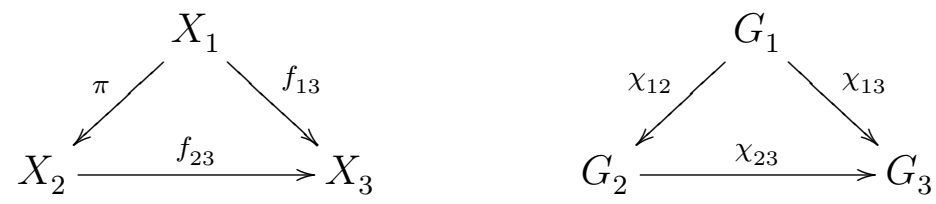

Demonstração: Dada $f_{13}$ e definindo $f_{23}$ por $f_{13}=f_{23} \circ \pi$, vemos que $f_{23}$ é de fato uma aplicação bem definida, pois $\pi$ é sobrejetora e $\pi\left(m^{\prime}\right)=q=\pi(m)$ implica $m^{\prime}=k \cdot m$ para algum $k \in K_{12}$ (já que $\pi^{-1}(q)$ é uma $K_{12}$-órbita), implicando que $f_{13}\left(m^{\prime}\right)=f_{13}(k \cdot m)=$ $\chi_{12}(k) \cdot f_{13}(m)=f_{13}(m)$. Além disso, como $\pi$ é uma submersão, temos que se $f_{13}$ é de classe $C^{\infty}$, então $f_{23}$ também o é.

\subsection{Acoplamento mínimo}

Consideramos inicialmente campos de matéria em conjunto com campos de calibre externos, caso que foi tratado em [4], porém apenas para campos de matéria lineares (seções de fibrados vetoriais) e com uma abordagem distinta da nossa, que não trata transformações de calibre em um contexto de dimensão finita e, a nosso ver, não lida com a noção correta de invariância global. 
Seja $E$ um fibrado com grupo estrutural $G$, que desempenha o papel de fibrado de configuração para os campos de matéria da teoria em questão (o fibrado cujas seções representam os campos de matéria da teoria), e seja $P$ o fibrado principal sobre $M$ com grupo estrutural $G$ ao qual $E$ é associado, de modo que $E=P \times_{G} Q$ onde $Q$ é a fibra típica de $E$ na qual $G$ age à esquerda. Nesta situação, temos como ponto de partida para nossa discussão de simetrias a ação (3.38) do fibrado de grupos de Lie $P \times_{G} G$ no fibrado de configuração $P \times{ }_{G} Q$, que escreveremos na forma

$$
\begin{aligned}
& \left(P \times_{G} G\right) \times_{M}\left(P \times_{G} Q\right) \longrightarrow P \times_{G} Q \\
& (g, \varphi) \quad \longmapsto g \cdot \varphi
\end{aligned}
$$

Conforme a Proposição 3.1, ela induz uma ação do mesmo fibrado de grupos de Lie $P \times{ }_{G} G$ no fibrado de jatos linearizado $\vec{J}\left(P \times_{G} Q\right)$, que escreveremos na forma

$$
\begin{aligned}
& \left(P \times_{G} G\right) \times_{M} \vec{J}\left(P \times_{G} Q\right) \longrightarrow \vec{J}\left(P \times_{G} Q\right) \\
& (g,(\varphi, D \varphi)) \quad \longmapsto g \cdot(\varphi, D \varphi)
\end{aligned}
$$

e conforme o Teorema 3.2, também induz uma ação do fibrado de grupos de Lie $J\left(P \times_{G} G\right)$ no fibrado de jatos $J\left(P \times_{G} Q\right)$, que escreveremos na forma

$$
\begin{array}{ccc}
J\left(P \times_{G} G\right) \times_{M} J\left(P \times_{G} Q\right) & \longrightarrow & J\left(P \times_{G} Q\right) \\
((g, \partial g),(\varphi, \partial \varphi)) & \longmapsto & (g, \partial g) \cdot(\varphi, \partial \varphi)
\end{array}
$$

Por outro lado, temos a ação (3.71) do fibrado de grupos de Lie $J\left(P \times_{G} G\right)$ no fibrado das conexões $C P$ de $P$, que escreveremos na forma

$$
\begin{array}{ccc}
J\left(P \times_{G} G\right) \times{ }_{M} C P & \longrightarrow & C P \\
((g, \partial g), A) & \longmapsto(g, \partial g) \cdot A
\end{array} .
$$

Juntando estas duas, obtemos uma ação do fibrado de grupos de Lie $J\left(P \times_{G} G\right)$ no produto em fibras de $J\left(P \times{ }_{G} Q\right)$ e $C P$ :

$$
\begin{array}{ccc}
J\left(P \times_{G} G\right) \times_{M}\left(C P \times_{M} J\left(P \times_{G} Q\right)\right) & \longrightarrow & C P \times_{M} J\left(P \times_{G} Q\right) \\
((g, \partial g),(A,(\varphi, \partial \varphi))) & \longmapsto & ((g, \partial g) \cdot A,(g, \partial g) \cdot(\varphi, \partial \varphi))
\end{array}
$$

Agora consideramos lagrangianas de primeira ordem para tais campos de matéria acoplados a um campo de calibre não dinâmico, que são funções (mais exatamente, homomorfismos de fibrados sobre $M$ )

$$
\mathcal{L}_{\text {mat }}: C P \times_{M} J\left(P \times_{G} Q\right) \longrightarrow \bigwedge^{n} T^{*} M,
$$


e supomos que elas sejam invariantes sob transformações de calibre locais, ou seja, invariantes sob a ação do fibrado de grupos de Lie $J\left(P \times_{G} G\right)$. Por outro lado, consideramos lagrangianas reduzidas, que nesta mesma situação são definidas como funções (mais exatamente, homomorfismos de fibrados sobre $M$ )

$$
\mathcal{L}_{\text {mat }}^{\text {red }}: \vec{J}\left(P \times_{G} Q\right) \longrightarrow \bigwedge^{n} T^{*} M,
$$

supondo que elas sejam invariantes sob transformações de calibre globais, ou seja, invariantes sob a ação do fibrado de grupos de Lie $P \times_{G} G$. Nossa meta nessa seção será provar que existe uma relação biunívoca entre estes dois tipos de lagrangiana, que é estabelecida pela aplicação derivada covariante universal $\mathcal{D}: C P \times_{M} J\left(P \times_{G} Q\right) \rightarrow \vec{J}\left(P \times_{G} Q\right)$ introduzida na Seção 2.3.4 (veja a equação (2.145)):

$$
\mathcal{L}_{\text {mat }}=\mathcal{L}_{\text {mat }}^{\text {red }} \circ \mathcal{D} .
$$

Primeiro, notamos uma propriedade crucial desta aplicação: ela é equivariante em relação às ações dos fibrados de grupos de Lie $J\left(P \times_{G} G\right)$ em $C P \times_{M} J\left(P \times_{G} Q\right)$ e $P \times_{G} G$ em $\vec{J}\left(P \times_{G} Q\right)$ que, por sua vez, estão relacionados pela projeção alvo

$$
J\left(P \times_{G} G\right) \longrightarrow P \times_{G} G,
$$

vista como homomorfismo de fibrados de grupos de Lie, ou seja, temos os seguintes diagramas comutativos,

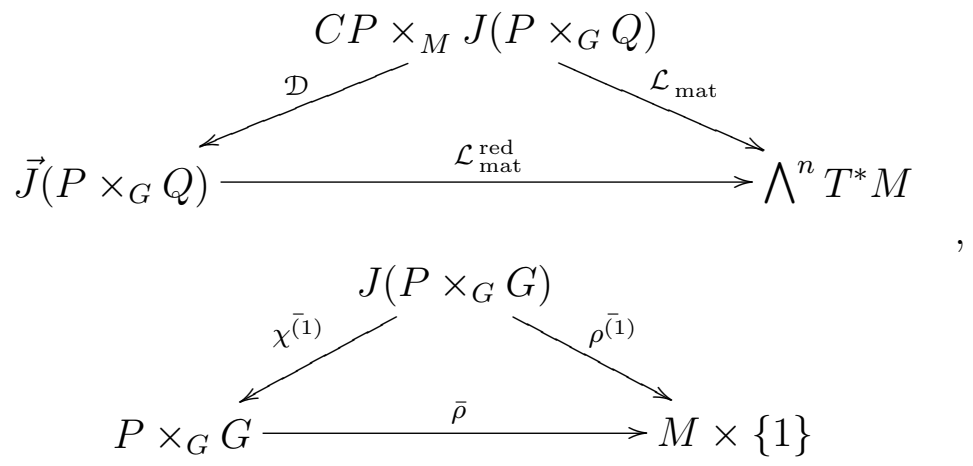

onde os fibrados de grupo de Lie do diagrama de baixo agem nos fibrados do diagrama de cima, e de maneira equivariante em relação às respectivas projeções.

Isso pode ser mais facilmente verificado em termos de uma seção local $\sigma$ de $P$, usando as representações locais por ela induzidas em todos os fibrados envolvidos (porém sem empregar uma carta local de $M$, o que simplifica as fórmulas),

$$
\begin{aligned}
C \Phi_{\sigma}:\left.C P\right|_{U} & \left.\longrightarrow L(T M, M \times \mathfrak{g})\right|_{U} \\
A_{m} & \longmapsto\left(m, A_{m}^{\sigma}\right)
\end{aligned}
$$




$$
\begin{array}{cccc}
J \Phi_{\sigma}:\left.\quad J\left(P \times_{G} Q\right)\right|_{\pi^{-1}(U)} & \longrightarrow & \left.L\left(\operatorname{pr}_{1}^{*}(T M), \operatorname{pr}_{2}^{*}(T Q)\right)\right|_{U \times Q}, \\
j_{m} \varphi & \longmapsto & \left(m, T_{m} \varphi_{\sigma}\right) \\
\vec{J} \Phi_{\sigma}:\left.\quad \vec{J}\left(P \times_{G} Q\right)\right|_{\pi^{-1}(U)} & \longrightarrow & \left.L\left(\operatorname{pr}_{1}^{*}(T M), \operatorname{pr}_{2}^{*}(T Q)\right)\right|_{U \times Q}, \\
\vec{j}_{m} \varphi & \longmapsto & \left(m, T_{m} \varphi_{\sigma}\right)
\end{array}
$$

(onde $\vec{J} \Phi_{\sigma}=\operatorname{id}_{T^{*} M} \otimes V \Phi_{\sigma}$ e $\left.\vec{J}\left(P \times_{G} Q\right)\right|_{U}=\left.T^{*} U \otimes V\left(P \times_{G} Q\right)\right|_{U}$ ), e

$$
\begin{aligned}
\Phi_{\sigma}:\left.\left(P \times_{G} G\right)\right|_{U} & \longrightarrow U \times G \\
g(m) & \longmapsto\left(m, g_{\sigma}(m)\right) \\
J \Phi_{\sigma}:\left.\quad J\left(P \times_{G} G\right)\right|_{\pi^{-1}(U)} & \left.\longrightarrow L\left(\operatorname{pr}_{1}^{*}(T M), M \times G \ltimes \mathfrak{g}\right)\right|_{U \times G} \\
j_{m} g & \longmapsto\left(m, g_{\sigma}(m), T_{m} g_{\sigma} g_{\sigma}(m)^{-1}\right)
\end{aligned}
$$

de modo que, para cada ponto $m$ de $U$, obtemos diagramas comutativos

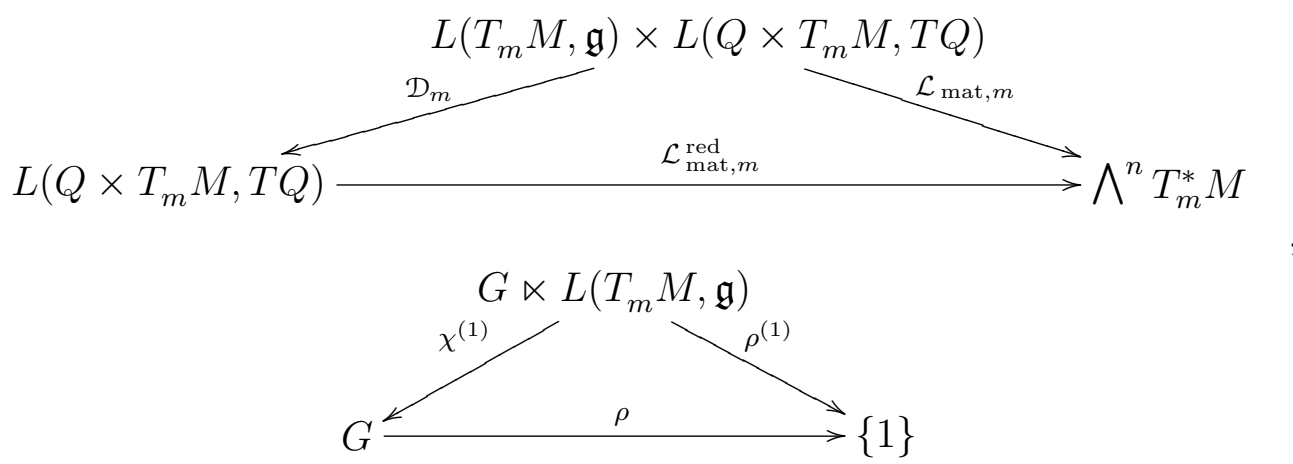

onde, conforme a equação (2.143), a aplicação $\mathcal{D}_{m}$ é dada por

$$
\begin{aligned}
\mathcal{D}_{m}: L\left(T_{m} M, \mathfrak{g}\right) \times L\left(Q \times T_{m} M, T Q\right) & \longrightarrow L\left(Q \times T_{m} M, T Q\right), \\
\left(\alpha_{m}, v_{q}\right) & \longmapsto v_{q}+\left(\alpha_{m}\right)_{Q}(q)
\end{aligned}
$$

e onde os grupos de Lie do diagrama de baixo agem nas variedades do diagrama de cima, e de maneira equivariante em relação às respectivas projeções, conforme as seguintes regras: a ação de $G \ltimes L\left(T_{m} M, \mathfrak{g}\right)$ em $L\left(T_{m} M, \mathfrak{g}\right)$ é dada pela equação (3.73),

$$
\left(g_{\sigma}, \partial g_{\sigma}\right) \cdot A^{\sigma}=g_{\sigma} A^{\sigma} g_{\sigma}^{-1}-\partial g_{\sigma} g_{\sigma}^{-1},
$$

enquanto que as ações de $G \ltimes L\left(T_{m} M, \mathfrak{g}\right)$ e de $G$ em $L\left(Q \times T_{m} M, T Q\right.$ são obtidas por diferenciação da ação de $G$ em $Q$ usando a definição do campo fundamental de uma ação e a regra do produto,

$$
\left(g_{\sigma}, \partial g_{\sigma}\right) \cdot\left(\varphi_{\sigma}, \partial \varphi_{\sigma}\right)=\left(g_{\sigma} \cdot \varphi_{\sigma}, g_{\sigma} \cdot \partial \varphi_{\sigma}+\left(\partial g_{\sigma} g_{\sigma}^{-1}\right)_{Q} \circ\left(g_{\sigma} \cdot \varphi_{\sigma}\right)\right)
$$




$$
g_{\sigma} \cdot\left(\varphi_{\sigma}, D \varphi_{\sigma}\right)=\left(g_{\sigma} \cdot \varphi_{\sigma}, g_{\sigma} \cdot D \varphi_{\sigma}\right)
$$

Aplicando mais uma vez a equação (2.143), ou seja,

$$
D^{A^{\sigma}} \varphi_{\sigma}=\partial \varphi_{\sigma}+\left(A^{\sigma}\right)_{Q} \circ \varphi_{\sigma}
$$

isso demonstra a equivariância da aplicação $\mathcal{D}_{m}$ e assim, da aplicação $\mathcal{D}$. Logo, compondo uma lagrangiana reduzida $\mathcal{L}_{\text {mat }}^{\text {red }}$ globalmente invariante com $\mathcal{D}$ vai produzir uma lagrangiana $\mathcal{L}_{\text {mat }}$ plena localmente invariante.

Definição 4.1 Dada uma lagrangiana reduzida $\mathcal{L}_{\text {mat }}^{\text {red }}$ globalmente invariante, a lagrangiana $\mathcal{L}_{\text {mat }}$ plena localmente invariante obtida por composição com $\mathcal{D}$,

$$
\mathcal{L}_{\text {mat }}=\mathcal{L}_{\text {mat }}^{\text {red }} \circ \mathcal{D}
$$

é dita ser obtida a partir dela por acoplamento mínimo.

Reciprocamente, observamos que nos deparamos exatamente com o contexto colocado nas hipóteses da Proposição 4.1, com

$$
\begin{gathered}
X_{1}=L\left(T_{m} M, \mathfrak{g}\right) \times L\left(Q \times T_{m} M, T Q\right), \quad X_{2}=L\left(Q \times T_{m} M, T Q\right), X_{3}=\bigwedge^{n} T_{m} M, \\
\pi=\mathcal{D}_{m}, f_{13}=\mathcal{L}_{\text {mat }, m}, f_{23}=\mathcal{L}_{\text {mat }, m}^{\text {red }}, \\
G_{1}=G \ltimes L\left(T_{m} M, \mathfrak{g}\right), G_{2}=G, G_{3}=\{1\} \\
\chi_{12}=\operatorname{pr}_{1}, \chi_{13} \text { trivial }, \chi_{23} \text { trival }, K_{12}=\operatorname{ker} \chi_{12}=L\left(T_{m} M, \mathfrak{g}\right) .
\end{gathered}
$$

Agora é óbvio que $\mathcal{D}_{m}$ é uma aplicação afim sobrejetora e portanto uma submersão sobrejetora que é $\chi_{12}$-equivariante e que $K_{12}=L\left(T_{m} M, \mathfrak{g}\right)$ age transitivamente nas suas fibras, pois estas consistem no próprio espaço $L\left(T_{m} M, \mathfrak{g}\right)$, no qual $K_{12}$ age aditivamente. Assim, todas as hipóteses da Proposição 4.1 estão satisfeitas, o que nos permite concluir que:

Teorema 4.1 Uma lagrangiana $\mathcal{L}_{\text {mat }}: C P \times_{M} J\left(P \times_{G} Q\right) \rightarrow \bigwedge^{n} T^{*} M$ é localmente invariante (invariante sob a ação de $J\left(P \times_{G} G\right)$ ) se e somente se existe uma lagrangiana reduzida $\mathcal{L}_{\text {mat }}^{\text {red }}: \vec{J}\left(P \times_{G} Q\right) \rightarrow \bigwedge^{n} T^{*} M$ que é globalmente invariante (invariante sob a ação de $P \times_{G} G$ ) da qual ela é obtida por acoplamento mínimo.

\subsection{Teorema de Utiyama}

Passamos agora ao procedimento análogo ao do acoplamento mínimo para o caso das teorias de calibre puras, isto é, teorias que descrevem a dinâmica dos próprios campos de calibre (e 
apenas deles). Consideramos então lagrangianas de primeira ordem para campos de calibre dinâmicos, que são funções (mais exatamente, homomorfismos de fibrados sobre $M$ )

$$
\mathcal{L}_{\text {cal }}: J(C P) \longrightarrow \bigwedge^{n} T^{*} M,
$$

e supomos que elas sejam invariantes sob transformações de calibre locais, ou seja, invariantes sob a ação do fibrado de grupos de Lie $J^{2}\left(P \times_{G} G\right)$. Por outro lado, consideramos lagrangianas reduzidas, que nesta mesma situação são definidas como funções (mais exatamente, homomorfismos de fibrados sobre $M$ )

$$
\mathcal{L}_{\text {cal }}^{\text {red }}: \bigwedge^{2} T^{*} M \otimes\left(P \times_{G} \mathfrak{g}\right) \longrightarrow \bigwedge^{n} T^{*} M,
$$

supondo que elas sejam invariantes sob transformações de calibre globais, ou seja, invariantes sob a ação do fibrado de grupos de Lie $P \times_{G} G$. Nossa meta nessa seção será provar o teorema de Utiyama, segundo o qual existe uma relação biunívoca entre estes dois tipos de lagrangiana, que é estabelecida pela aplicação curvatura universal $\mathcal{F}: J(C P) \rightarrow \bigwedge^{2} T^{*} M \otimes$ $\left(P \times_{G} \mathfrak{g}\right)$ introduzida na Seção 2.3.5 (veja a equação (2.151)):

$$
\mathcal{L}_{\text {cal }}=\mathcal{L}_{\text {cal }}^{\text {red } \circ \mathcal{F}} .
$$

Novamente, começamos notando uma propriedade crucial desta aplicação: ela é equivariante em relação às ações dos fibrados de grupos de Lie $J^{2}\left(P \times_{G} G\right)$ em $J(C P)$ e $P \times_{G} G$ em $P \times_{G} \mathfrak{g}$ que, por sua vez, estão relacionados pela projeção natural

$$
J^{2}\left(P \times_{G} G\right) \longrightarrow P \times_{G} G,
$$

vista como homomorfismo de fibrados de grupos de Lie, ou seja, temos os seguintes diagramas comutativos,

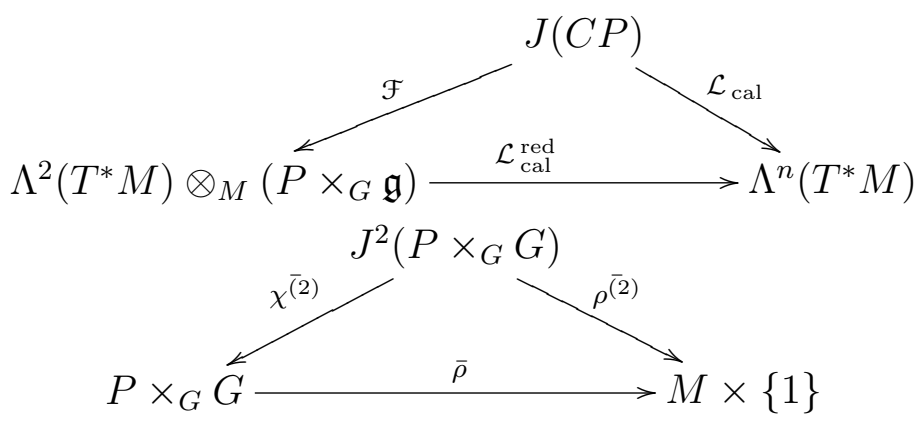

Em termos de uma seção local $\sigma$ de $P$, usando as representações locais induzidas por ela em todos os fibrados envolvidos,

$$
J(C \Phi)_{\sigma}:\left.\quad J(C P)\right|_{U} \longrightarrow U \times L\left(T_{m} M, \mathfrak{g}\right) \times L^{2}\left(T_{m} M, \mathfrak{g}\right)
$$


e

$$
\Phi_{\sigma}:\left.\Lambda^{2}\left(T^{*} M\right) \otimes_{M}\left(P \times_{G} \mathfrak{g}\right)\right|_{U} \longrightarrow U \times \Lambda^{2}\left(T_{m} M\right)^{*} \otimes \mathfrak{g},
$$

temos que a hipótese de que a lagrangiana é invariante sob transformações de calibre juntamente com o fato (que provaremos abaixo) de que a curvatura é equivariante em relação a elas implicam que a curvatura e as lagrangianas definem no ponto $m$ as funções

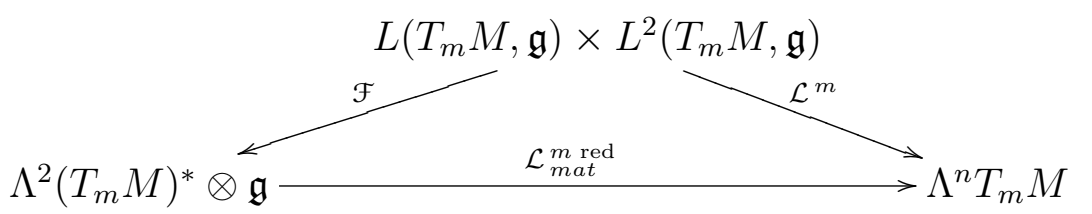

Por outro lado, usando representações locais compatíveis

$$
\begin{aligned}
& \Phi_{\sigma}:\left.\left(P \times_{G} G\right)\right|_{U} \longrightarrow U \times G \\
& g(m) \longmapsto\left(m, g_{\sigma}(m)\right)
\end{aligned}
$$

$\mathrm{e}$

$$
\begin{aligned}
J^{2} \Phi_{\sigma}^{x}:\left.J^{2}\left(P \times_{G} G\right)\right|_{U} & \longrightarrow U \times J_{0}^{2}\left(\mathbb{R}^{n}, G\right) \\
j_{m}^{2} g & \longmapsto\left(m, j_{0}^{2}\left(g_{\sigma} \circ x^{-1}\right)\right),
\end{aligned}
$$

obtemos nas fibras dos fibrados de grupos de Lie em (4.7) o seguinte diagrama:

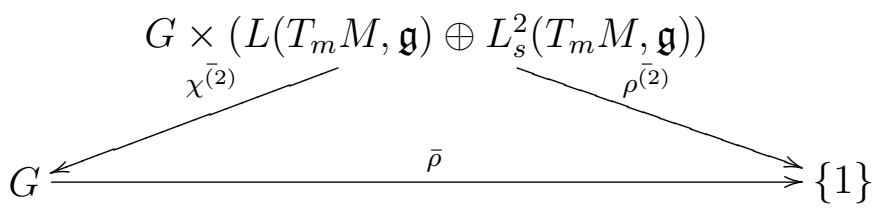

Lema 4.1 A aplicação

$$
\begin{array}{ccc}
\mathcal{F}: J(C P) & \longrightarrow & \Lambda^{2}\left(T^{*} M\right) \otimes_{M}\left(P \times_{G} \mathfrak{g}\right) \\
j_{m} A & \longmapsto & \mathcal{D}\left(j_{m} A, A(m)\right)
\end{array}
$$

induz, nas representações locais destes fibrados, a submersão sobrejetora $\chi^{(2)}$-equivariante :

$$
\begin{aligned}
\mathcal{F}^{\prime}: L\left(T_{m} M, \mathfrak{g}\right) \times L^{2}\left(T_{m} M, \mathfrak{g}\right) & \longrightarrow \Lambda^{2}\left(T_{m} M\right)^{*} \otimes \mathfrak{g} \\
(\alpha, \beta) & \longmapsto\left(\beta_{a s}+[\alpha, \alpha]_{\wedge}\right)
\end{aligned},
$$

onde $\chi^{(2)}$ é a projeção de $G \times\left(L\left(T_{m} M, \mathfrak{g}\right) \oplus L_{s}^{2}\left(T_{m} M, \mathfrak{g}\right)\right)$ em $G$. 
Novamente, nos deparamos exatamente com o contexto colocado nas hipóteses da Proposição 4.1, com

$$
\begin{gathered}
X_{1}=L\left(T_{m} M, \mathfrak{g}\right) \times L^{2}\left(T_{m} M, \mathfrak{g}\right), \quad X_{2}=\Lambda^{2}\left(T_{m} M\right)^{*} \otimes \mathfrak{g}, \quad X_{3}=\Lambda^{n} T_{m} M, \\
\pi=\mathcal{F}, \quad f_{13}=\mathcal{L}^{m}, f_{23}=\mathcal{L}_{\text {cal }}^{m \text { red }}, \\
G_{1}=G \times\left(L\left(T_{m} M, \mathfrak{g}\right) \oplus L_{s}^{2}\left(T_{m} M, \mathfrak{g}\right)\right), \quad G_{2}=G, \quad G_{3}=\{1\}, \\
\left.\chi_{12}=\chi^{(2)}, \quad \chi_{13}=\rho^{(2)}, \quad \chi_{23}=\rho, \quad K_{12}=\operatorname{ker} \chi^{(2)}=L\left(T_{m} M, \mathfrak{g}\right) \oplus L_{s}^{2}\left(T_{m} M, \mathfrak{g}\right)\right) .
\end{gathered}
$$

Neste caso, as fibras de $\mathcal{F}$ consistem em $L\left(T_{m} M, \mathfrak{g}\right) \times L_{s}^{2}\left(T_{m} M, \mathfrak{g}\right)$, ou seja, novamente coincidem com $K_{12}$. A equação nos diz que este grupo age (de novo) aditivamente em $L\left(T_{m} M, \mathfrak{g}\right) \times L_{s}^{2}\left(T_{m} M, \mathfrak{g}\right)$, e portanto a ação é transitiva. Assim, todas as hipóteses da Proposição 4.1 estão satisfeitas, o que nos permite concluir que:

Teorema 4.2 (Utiyama) Uma Lagrangiana $\mathcal{L}_{\text {cal }}: J(C P) \rightarrow \Lambda^{n}\left(T^{*} M\right)$ é invariante sob a ação de $J^{2}\left(P \times_{G} G\right)$ se e somente se existe uma função

$$
\mathcal{L}_{\text {cal }}^{\text {red }}: \Lambda^{2}\left(T^{*} M\right) \otimes_{M}\left(P \times_{G} \mathfrak{g}\right) \rightarrow \Lambda^{n}\left(T^{*} M\right)
$$

invariante sob a ação de $P \times{ }_{G} G$ tal que o diagrama 4.7 comuta

Juntando os dois últimos resultados e considerando a aplicação

$$
\begin{array}{ccc}
\mathcal{F}^{+}: J\left(\left(P \times_{G} Q\right) \times_{M} C P\right) & \longrightarrow & \left(\vec{J}\left(P \times_{G} Q\right)\right) \times_{M}\left(\Lambda^{2}\left(T^{*} M\right) \otimes_{M}\left(P \times_{G} \mathfrak{g}\right)\right) \\
\left(j_{m} \phi, j_{m} A\right) & \longmapsto & \left(\mathcal{D}_{m}^{A} \phi, F^{A}(m)\right)
\end{array}
$$

temos:

Teorema 4.3 Uma Lagrangiana $\mathcal{L}: J\left(\left(P \times_{G} Q\right) \times{ }_{M} C P\right) \rightarrow \Lambda^{n}\left(T^{*} M\right)$ é invariante sob a ação de $J^{2}\left(P \times_{G} G\right)$ se e somente se existe uma função

$$
\mathcal{L}^{\text {red }}:\left(\vec{J}\left(P \times_{G} Q\right)\right) \times_{M}\left(\Lambda^{2}\left(T^{*} M\right) \otimes_{M}\left(P \times_{G} \mathfrak{g}\right)\right) \rightarrow \Lambda^{n}\left(T^{*} M\right)
$$

invariante sob a ação de $P \times_{G} G$ tal que

$$
\mathcal{L}=\mathcal{L}^{\text {red } \circ \mathcal{F}^{+}} .
$$





\section{Conclusão e perspectivas}

Nesta tese, implementamos o arcabouço geométrico necessário para se estudar sistemas com simetria em teoria dos campos. o principal introdução do conceito de ação de um fibrado de grupos de Lie em um fibrado e a análise de como ele funciona nos objetos de interesse da física geométrica..

Esperamos que a primeira contribuição que venha fornecer à geometria multissimplética é ser a base da obtenção de um teorema de redução de fibrados multissimpléticos. Para poder até mesmo formular este problema, era necessário primeiramente encontrar a definição adequada de variedade multissimplética, o que só foi feito bem recentemente em ( [17]).

Inclusive, neste trabalho já direcionamos o formalismo desenvolvido rumo ao formalismo hamiltoniano, pois aplicamos as ferramentas que construímos para dar uma demonstração com alguns elementos novos do teorema de Utiyama e do mecanismo de acoplamento mínimo.

\section{Aplicação momento}

Sejam $\pi: F \rightarrow M$ um fibrado geral sobre $M, \bar{G}$ um fibrado de grupos de Lie sobre $M, \overline{\mathfrak{g}}$ o correspondente fibrado de álgebras de Lie sobre $M$ com a aplicação exp $: \overline{\mathfrak{g}} \rightarrow \bar{G}$.

Recordamos que uma ação de $\bar{G}$ em $F$ define o homomorfismo de fibrados vetoriais (3.47) sobre $F$,

$$
\begin{aligned}
\pi^{*} \overline{\mathfrak{g}} & \rightarrow V F \\
X_{f} & \mapsto X_{f}^{F}
\end{aligned}
$$

Recordamos também que uma ação de $\bar{G}$ em $F$ induz ações de $\bar{G}$ em $\vec{J} F$ e, por dualização linear, em $\vec{J}{ }^{\circledast} F$, assim como ações de $J \bar{G}$ em $J F$ e, por dualização afim, em $J^{\circledast} F$. 
Se $E$ é o fibrado de configuração da teoria de campo em questão, então a aplicação momento definida pela ação de $P \times_{G} G$ em $\vec{J}{ }^{\circledast} E$ (induzida pela ação de $P \times_{G} G$ em $E$ ) é o homomorfismo de fibrados vetoriais que faz o seguinte diagrama comutar:
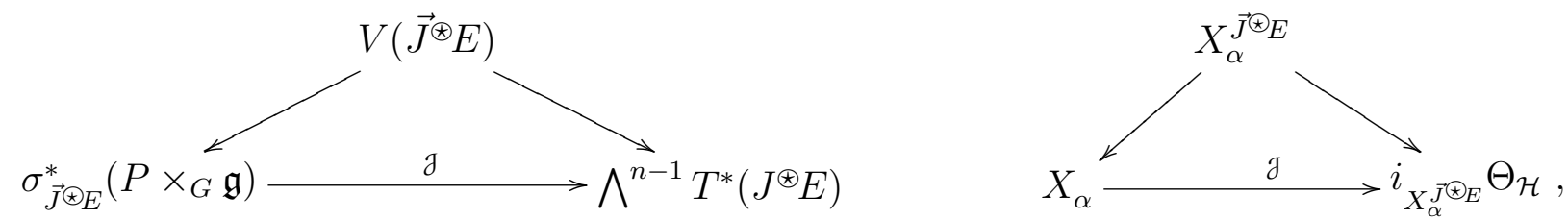

onde $\alpha \in \vec{J}^{\circledast} E$ e $\mathcal{H}$ é uma seção do fibrado $J^{\circledast} E$ visto como um fibrado de linhas sobre $\vec{J}^{\circledast} E$ (aqui, o fibrado $F$ considerado acima é $\sigma_{\vec{J}{ }^{\circledast E}}^{*}: \vec{J}^{\circledast} E \rightarrow M, \bar{G}$ é $P \times_{G} G$ e $\overline{\mathfrak{g}}$ é $P \times_{G} \mathfrak{g}$ ).

A construção acima não depende da hamiltoniana, pois $X_{\alpha}^{\vec{J}^{\circledast E}}$ é um campo vertical em $\vec{J} \circledast E$.

Para encontrarmos a aplicação momento no caso em que lidamos com simetrias locais, por sua vez, necessitamos primeiramente saber qual é o campo fundamental correspondente à acão (3.82) do grupo de Lie $G \ltimes L\left(\mathbb{R}^{n}, \mathfrak{g}\right)$. A álgebra de Lie deste grupo é a soma semidireta $\mathfrak{g} \ltimes L\left(\mathbb{R}^{n}, \mathfrak{g}\right)$, onde, em virtude da definição do produto desse grupo (fórmula ...), o comutador é dado por

$$
\begin{gathered}
{\left[\left(X_{1}, \xi_{1}\right),\left(X_{2}, \xi_{2}\right)\right]=\left(\left[X_{1}, X_{2}\right],\left[X_{1}, \xi_{2}\right]-\left[X_{2}, \xi_{1}\right]\right)} \\
\operatorname{para}\left(X_{1}, \xi_{1}\right),\left(X_{2}, \xi_{2}\right) \in \mathfrak{g} \ltimes L\left(\mathbb{R}^{n}, \mathfrak{g}\right) .
\end{gathered}
$$

Dado um elemento $(X, \xi)$ desta álgebra, deduzimos novamente a partir da definição do produto em $G \ltimes L\left(\mathbb{R}^{n}, \mathfrak{g}\right)$ que o campo invariante à esquerda definido por este elemento é dado por

$$
\overline{(X, \xi)}(g, \eta)=(g \cdot \xi,[\eta, \xi]+\eta)
$$

Temos então

$$
(X, \xi)^{\left(T^{n} Q\right)^{*} \times \mathbb{R}}\left(q, \alpha_{q}, p\right)=\left(X^{\left(T^{n} Q\right)^{*}}\left(q, \alpha_{q}\right),-\left\langle\alpha_{q}, \xi^{Q}(q)\right\rangle\right)
$$

Em termos de coordenadas locais $\left(q^{i}, p_{i}^{\mu}\right)$ de $\left(T^{n} Q\right)^{*}$ induzidas por coordenadas locais $q^{i}$ de $Q$, supondo que $X_{Q}$ e $\xi_{Q}$ assumem, respectivamente, as expressões locais $X_{Q}=X_{Q}^{i} \frac{\partial}{\partial q^{i}}$ e $\left(\xi_{Q}\right)_{\mu}=\left(\xi_{Q}\right)_{\mu}^{i} \frac{\partial}{\partial q^{i}}$

$$
(X, \xi)^{\left(T^{n} Q\right)^{*} \times \mathbb{R}}=X^{i} \frac{\partial}{\partial q^{i}}-p_{i}^{\mu} \frac{\partial X^{i}}{\partial q^{j}} \frac{\partial}{\partial p_{j}^{\mu}}-p_{i}^{\mu} \xi_{\mu}^{i} \frac{\partial}{\partial p} .
$$

Lembrando que a 1-forma canônica assume a expressão local

$$
\theta=p_{i}^{\mu} d q^{i} \wedge d^{n} x_{\mu}+p d^{n} x
$$


podemos ver que $L_{(X, \xi)^{\left(T^{n} Q\right)^{*} \times \mathbb{R}}} \theta=0$, pois

$$
\begin{aligned}
& i_{(X, \xi)^{\left(T^{n} Q\right)^{*} \times \mathbb{R}}} \theta=\quad p_{i}^{\mu} X^{i} d^{n} x_{\mu} \quad \Longrightarrow \\
& d i_{(X, \xi))^{\left(T^{n} Q\right)^{*} \times \mathbb{R}}} \theta=X^{i} d p_{i}^{\mu} \wedge d^{n} x_{\mu}+p_{i}^{\mu} \frac{\partial X^{i}}{\partial q^{j}} d q^{j} \wedge d^{n} x_{\mu}+p_{i}^{\mu} \frac{\partial X^{i}}{\partial x^{\mu}} d^{n} x,
\end{aligned}
$$

assim como

$$
i_{(X, \xi)^{T * Q \times \mathbb{R}}} d \theta=-X^{i} d p_{i}^{\mu} \wedge d^{n} x_{\mu}-p_{i}^{\mu} \frac{\partial X^{i}}{\partial q^{j}} d q^{j} \wedge d^{n} x_{\mu}-p_{i}^{\mu} \xi_{\mu}^{i} d^{n} x
$$

Uma vez que as coordenadas locais de $\xi^{Q}$ e $X^{Q}$ se relacionam pela fórmula $\xi_{\mu}^{i}=\frac{\partial X^{i}}{\partial x^{\mu}}$, concluímos que $L_{(X, \xi)^{T^{*} Q \times \mathbb{R}}} \theta=0$.

Isto nos permite concluir que a aplicação momento é dada por

$$
\begin{array}{ccc}
\mathcal{J}: \sigma_{J \otimes\left(P \times{ }_{G} Q\right)}^{*}\left(P \times_{G}(\mathfrak{g} \ltimes \mathfrak{g})\right) & \rightarrow \bigwedge^{n-1} T^{*}\left(J^{\circledast}\left(P \times_{G} Q\right)\right) \\
\langle\mathcal{J},(X, \xi)\rangle & \mapsto & i_{(X, \xi)^{\left(T^{n} Q\right)^{*} \times \mathbb{R}}} \theta .
\end{array}
$$

Observamos que apenas a primeira componente de $\mathfrak{g} \ltimes L\left(\mathbb{R}^{n}, \mathfrak{g}\right.$ ) (a parte global da ação) determina a aplicação momento, e portanto na verdade ela consiste em uma função

$$
\begin{array}{cl}
\mathcal{J}: \sigma_{J \otimes\left(P \times_{G} Q\right)}^{*}\left(P \times_{G} \mathfrak{g}\right) & \rightarrow \bigwedge^{n-1} T^{*}\left(J^{\circledast}\left(P \times_{G} Q\right)\right) \\
X & \mapsto\langle\mathcal{J}, X\rangle=X^{i} p_{i}^{\mu} d^{n} x_{\mu}
\end{array}
$$

que também pode ser interpretada como uma função

$$
\mathfrak{J}: J^{\circledast}\left(P \times_{G} Q\right) \rightarrow \sigma_{J \circledast\left(P \times_{G} Q\right)}^{*}\left(P \times_{G} \mathfrak{g}^{*}\right) \otimes_{M} \bigwedge^{n-1} T^{*}\left(J^{\circledast}\left(P \times_{G} Q\right)\right)
$$

de acordo com um procedimento análogo ao mencionado acima no caso da mecânica.

\section{Redução no fibrado de cojatos}

Generalizando a situação encontrada na mecânica, espera-se que, em teorias de calibre, a identificação do verdadeiro espaço de multifase requeira a passagem do nível zero da aplicação multimomento para um quociente apropriado. No entanto, não existe por enquanto nenhum procedimento de redução do espaço de multifase, isto é, nenhum análogo multissimplético do procedimento de redução do espaço de fase simplético à la Marsden-Weinstein, que consideremos satisfatório.

O problema da redução na teoria dos campos tem sido amplamente estudado na literatura, mas do ponto de vista da geometria multissimplética, foi abordado apenas recentemente, sob 
diferentes aspectos. Por exemplo, em $[8,10]$ realiza-se a redução no nível lagrangiano quando o fibrado de configuração é um fibrado principal. Uma outra direção, relacionada com o formalismo BRST e originalmente proposta em [7], é desenvolvida em [18], enquanto que em [25], a redução simplética é estendida a variedades polissimpléticas.

A aplicação momento definida na seção anterior se relaciona com a aplicação momento em variedades polissimpléticas definida por Munteanu et al. [25] construída a partir da forma polissimplética canônica em $T_{n}^{*} Q$ dada por $\sum_{i=1}^{n} \omega_{i} \otimes e_{i}$, onde $\omega_{i}$ é o pull-back da forma canônica $\omega$ em $T^{*} Q$ pela projeção $\tau_{i}$ de $T_{n}^{*} Q$ em sua $i$-ésima componente. Esta relação é interessante porque nos permite obter a versão multissimplética da redução simplética à la Marsden-Weinstein.

Se $S$ é uma variedade polissimplética em que está definida uma ação de um grupo de Lie $G$ com álgebra de Lie $\mathfrak{g}$, então a aplicação momento é uma função

$$
\mathcal{\partial}: S \longrightarrow\left(\mathfrak{g}^{n}\right)^{*}
$$

que satisfaz

$$
\sum_{j=1}^{n} i_{\left(\xi_{j}\right)_{S}} \omega_{j}=d \mathcal{J}_{\left(\xi_{1}, \ldots, \xi_{n}\right)} \quad \forall\left(\xi_{1}, \ldots, \xi_{n}\right) \in \mathfrak{g}^{n}, \quad \text { onde } \mathcal{J}_{\left(\xi_{1}, \ldots, \xi_{n}\right)}(x)=\left\langle\mathcal{J}(x),\left(\xi_{1}, \ldots, \xi_{n}\right)\right\rangle \text {. }
$$

Existe um análogo direto da redução simplética para variedades polissimpléticas:

Teorema 4.4 Seja J uma aplicação momento equivariante em uma variedade polissimplética $\left(S, \omega_{1}, \ldots, \omega_{n}\right)$, seja $\delta \in\left(\mathfrak{g}^{n}\right)^{*}$ um valor regular de $\mathcal{J}$ e $G_{\delta}$ o grupo de isotropia da ação coadjunta de $G$ em $\left(\mathfrak{g}^{n}\right)^{*}$. Suponha que $G_{\delta}$ age livre e propriamente em $\mathfrak{J}^{-1}(\delta)$. Então $S_{\delta}=\mathcal{J}^{-1}(\delta) / G_{\delta}$ tem uma única estrutura polissimplética $\left(\left(\omega_{\delta}\right)_{1}, \ldots,\left(\omega_{\delta}\right)_{n}\right)$ com a propriedade

$$
\pi_{\delta}^{*}\left(\omega_{\delta}\right)_{j}=i_{\delta}^{*} \omega_{j}, \quad 1 \leq j \leq n
$$

onde $\pi_{\delta}: \mathcal{J}^{-1}(\delta) \rightarrow S_{\delta}$ é a projeção no quociente e $i_{\delta}: \mathcal{J}^{-1}(\delta) \rightarrow S$ é a inclusão.

$$
\mathcal{J}: J^{\circledast}\left(P \times_{G} Q\right) \rightarrow \overrightarrow{J^{*}}\left(P \times_{G} G\right) / G \otimes_{M} \wedge^{n}\left(J^{\circledast}\left(P \times_{G} Q\right)\right)
$$

A idéia de redução fibra a fibra a partir da ação de um grupóide também aparece em [24] num contexto levemente diferente.

Concluindo, parece-nos que para resolver em definitivo as questões da definição correta de aplicação multimomento e da redução multissimplética, os principais passos consistem em: 
- Entender primeiramente o caso dos automorfismos estritos. A principal tarefa consiste em estender os resultados de Munteanu et al. da fibra típica para todo o fibrado dos cojatos,

- Estender os resultados a automorfismos não estritos. Aqui o conceito de ação de um grupóide de Lie em um fibrado terá um papel crucial.

Ainda seguindo a analogia com a mecânica, esperamos que tal procedimento de redução forneça variedades multissimpléticas mais gerais que fibrados de cojatos, que até o presente constituem a única classe de exemplos conhecida (veja o item seguinte). 



\section{Bibliografia}

[1] R. Abraham \& J.E. Marsden: Foundations of Mechanics, $2^{\text {nd }}$ edition, Benjamin/Cummings, Reading 1978.

[2] R. Abraham \& J.W. Robbin: Transversal Mappings and Flows, Benjamin/Cummings, Reading 1967.

[3] V.I. Arnold: Mathematical Methods of Classical Mechanics, $3^{\text {rd }}$ edition, Springer, Berlin 1978.

[4] D. Betounes: The Geometry of Gauge - Particle Field Interaction: A Generalization of Utiyama's Theorem, J. Geom. Phys. 6 (1989) 107-125.

[5] D. Bleecker: Gauge Theory and Variational Principles, Addison-Wesley, Reading 1981.

[6] V. Chari \& A. Pressley: A Guide to Quantum Groups, Cambridge University Press, Cambridge 1994.

[7] F. Cantrijn, A. Ibort \& M. De León: On the Geometry of Multisymplectic Manifolds, J. Austral. Math. Soc. (Series A) 66 (1999) 303-330.

[8] M. Castrillón López \& J.E. Marsden: Some Remarks on Lagrangian and Poisson Reduction for Field Theories, J. Geom. Phys. 48 (2003) 52-83.

[9] M. Castrillón López \& J. Muñoz Masqué: The Geometry of the Bundle of Connections, Math. Z. 236 (2001) 797-811.

[10] M. Castrillón López \& T.S. Ratiu: Reduction in Principal Bundles: Covariant LagrangePoincaré Equations, Commun. Math. Phys. 236 (2003) 223-250. 
[11] M. Daniel \& C.M. Viallet: The Geometrical Setting of Gauge Theories of the Yang-Mills Type, Rev. Mod. Phys. 52 (1980) 175-197.

[12] D.J. Eck: Gauge-Natural Bundles and Generalized Gauge Theories, Mem. Amer. Math. Soc. 247 (1981).

[13] M. Forger \& J. Kellendonk: Classical BRST Cohomology and Invariant Functions on Constraint Manifolds I, Commun. Math. Phys. 143 (1992) 235-251.

[14] P.L. García: Gauge Algebras, Curvature and Symplectic Geometry, J. Diff. Geom. 12 (1977) 209-227.

[15] W.H. Greub, S. Halperin \& R. Vanstone: Connections, Curvature and Cohomology, Vol. I, Academic Press, New York 1972, Vol. II, Academic Press, New York 1973, Vol. III, Academic Press, New York 1976.

[16] M. Gell-Mann \& Y. Ne'eman: The Eightfold Way, Benjamin, New York 1964.

[17] L.G. Gomes: Estruturas Polissimpléticas e Multissimpléticas em Variedades e Fibrados, Tese de Doutorado, IME-USP, Fevereiro de 2007.

[18] S.P. Hrabak: On a Multisymplectic Formulation of the Classical BRST Symmetry for First Order Field Theories, Part I: Algebraic Structures, preprint math-ph/9901012.

[19] I. Kolár, P.W. Michor \& J. Slovák: Natural Operations in Differential Geometry, Springer, Berlin 1993.

[20] S. Kobayashi \& K. Nomizu: Foundations of Differential Geometry, Vol. I, WileyInterscience, New York 1963, Vol. II, Wiley-Interscience, New York 1969.

[21] K.B. Marathe \& G. Martucci: The Geometry of Gauge Fields, J. Geom. Phys. 16 (1989) $1-106$.

[22] L. Mangiarotti \& M. Modugno: On the Geometric Structure of Gauge Theories, J. Math. Phys. 26 (1985) 1373-1379.

[23] L. Mangiarotti \& G. Sardanashvily: Connections in Classical and Quantum Field Theory, World Scientific, Singapore 2000.

[24] K. Mikami \& A. Weinstein: Moment Maps and Reduction for Symplectic Groupoids, Publ. RIMS Kyoto Univ. 24 (1988) 121-140.

[25] F. Munteanu, A.M. Rey \& M. Salgado: The Günther's Formalism in Classical Field Theory: Momentum Map and Reduction, J. Math. Phys. 45 (2004) 1730-1751. 
[26] G. Naber: Topology, Geometry and Gauge Fields - Interactions, Springer, Berlin 2000.

[27] D.J. Saunders: The Geometry of Jet Bundles, Cambridge University Press, Cambridge 1989.

[28] M. Socolovsky: Gauge Transformations and Fiber Bundle Theory, J. Math. Phys. 32 (1991) 2522-2531.

[29] N. Steenrod: The Topology of Fibre Bundles, Princeton University Press, Princeton 1951.

[30] J.M. Souriau: Structure des Systèmes Dynamiques, Dunod, Paris 1970.

[31] R. Utiyama: Invariant Theoretical Interpretation of Interaction, Phys. Rev. 101 (1956) 1597-1607.

[32] B.L. van der Waerden: Die Gruppentheoretische Methode in der Quantenmechanik, Springer, Berlin 1932; tradução revisada: Group Theory and Quantum Mechanics, Springer, Berlin 1974.

[33] H. Weyl: Gruppentheorie und Quantenmechanik, Hirzel, Leipzig 1931; tradução: The Theory of Groups and Quantum Mechanics, Dover, New York 1950.

[34] H. Weyl: The Classical Groups, their Invariants and Representations, Princeton University Press, Princeton 1939.

[35] H. Weyl: Gravitation und Elektrizität, Sitzungsber. Preuss. Akad. Wiss. Berlin 26 (1918) $465-480$.

[36] E.P. Wigner: Gruppentheorie und ihre Anwendung auf die Quantenmechanik der Atomespektren, Vieweg, Braunschweig 1991.

[37] E.P. Wigner: On Unitary Representations of the Inhomogeneous Lorentz Group, Ann. Math. 40 (1939) 149-204.

[38] C.N. Yang \& R.L. Mills: Conservation of Isotopic Spin and Isotopic Gauge Invariance, Phys. Rev. 96 (1954) 191-195. 